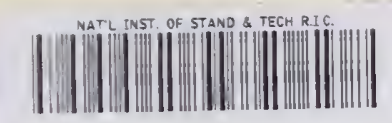

$A 11103756309$

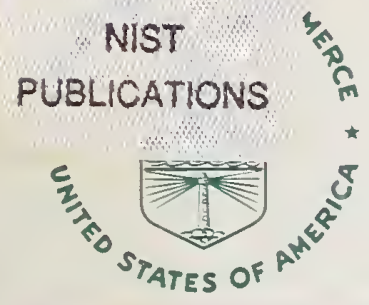

NIST SPECIAL PUBLICATION 400-90

U.S. DEPARTMENT OF COMMERCE/Technology Administration National Institute of Standards and Technology

Semiconductor Measurement Technology: \title{
Evaluating a Chip, Wafer, or Lot Using
SUXNS, SPICE, and STAT2 Evaluating a Chip, Wafer, or Lot Using
SUXNS, SPICE, and STAT2
}

$-Q C$
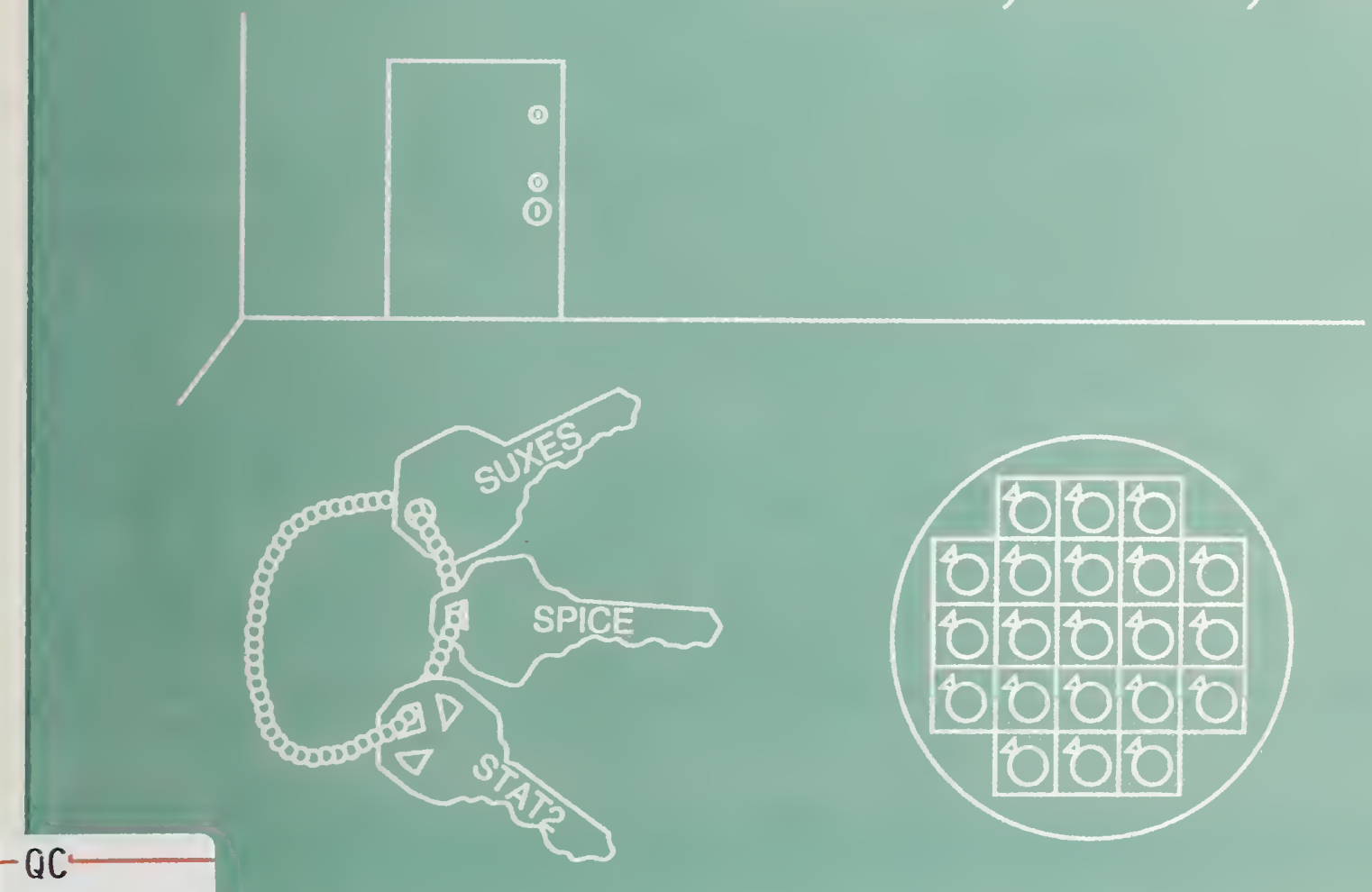

100

.457

400-90

1992

C. 2

J. C. Marshall and R. L. Mattis 


\section{DATE DUE}

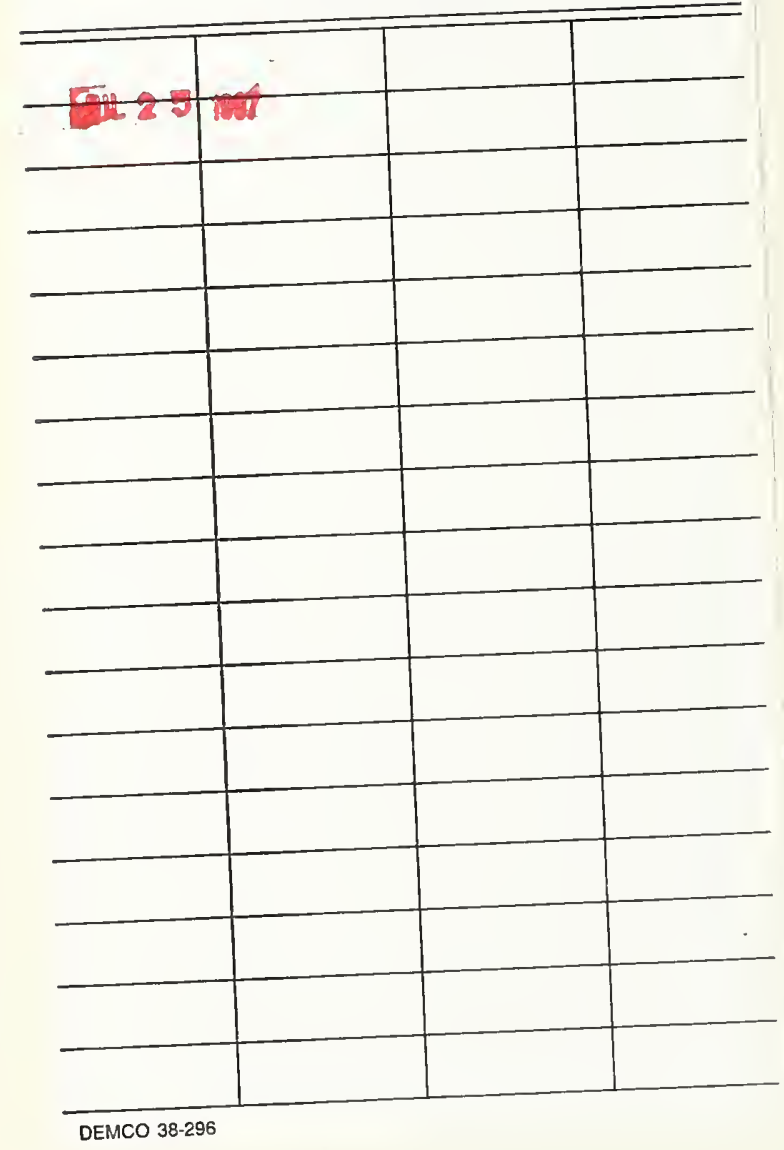


Semiconductor Measurement Technology:

\section{Evaluating a Chip, Wafer, or Lot Using SUXES, SPICE, and STAT2}

J. C. Marshall and R. L. Mattis

Semiconductor Electronics Division Electronics and Electrical Engineering Laboratory National Institute of Standards and Technology Gaithersburg, MD 20899

April 1992

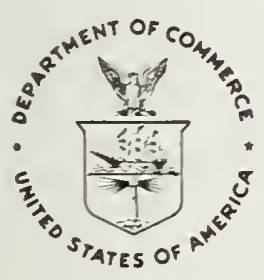

U.S. DEPARTMENT OF COMMERCE, Barbara Hackman Franklin, Secretary TECHNOLOGY ADMINISTRATION, Robert M. White, Under Secretary for Technology NATIONAL INSTITUTE OF STANDARDS AND TECHNOLOGY, John W. Lyons, Director 
National Institute of Standards and Technology Special Publication 400-90 Natl. Inst. Stand. Technol. Spec. Publ. 400-90, 145 pages (Apr. 1992) CODEN: NSPUE2

\section{U.S. GOVERNMENT PRINTING OFFICE}

WASHINGTON: 1992

For sale by the Superintendent of Documents, U.S. Government Printing Office, Washington, DC 20402-9325 


\section{TABLE OF CONTENTS}

Page

Abstract . . . . . . . . . . . . . . . . . 1

Nomenclature . . . . . . . . . . . . . . . . 2

1. Introduction . . . . . . . . . . . . . . . . . . 3

2. Motivation and General Principles . . . . . . . . . . . . . . . 7

2.1 Linking SUXES, SPICE, and STAT2 . . . . . . . . . . . . . 7

2.2 SUXES . . . . . . . . . . . . . . . . 8

$2.3 \mathrm{SPICE}$. . . . . . . . . . . . . . . . 10

2.4 STAT2 . . . . . . . . . . . . . . . . 20

3. An Example Using CMOS Technology . . . . . . . . . . . . . . . 27

3.1 Test Structure Measurement Methods . . . . . . . . . . . . . . . 27

3.2 The Output . . . . . . . . . . . . . . . . . . 30

3.2.1 The Output Plots . . . . . . . . . . . . . . . . . . 30

3.2.2 The Tabular Listings . . . . . . . . . . . . . . . . . . 36

3.2.3 The Wafer Maps . . . . . . . . . . . . . . . . . . . . . . 39

4. Conclusions . . . . . . . . . . . . . . . . . . . . 39

5. Acknowledgments . . . . . . . . . . . . . . . . . 40

6. References . . . . . . . . . . . . . . . . . . . 4 41

Appendix A - The Program Listing for the Computer Procedure KEYS . . . . . . 43

Appendix B - Modifying SUXES's Strategy File . . . . . . . . . . . . . . 131

Appendix C - The Procedure to Run KEYS . . . . . . . . . . . . . . . . . 133

\section{LIST OF FIGURES}

Page

1. Simplified block diagram for the computer procedure KEYS . . . . . . . . . 4

2. Flowchart for the computer procedure KEYS . . . . . . . . . . . . . . . . 5

3. Schematic grid for STAT2 nomenclature regarding site locations . . . . . . . . 23

4. Wafer map produced by STAT2 of the measured ring oscillator frequencies $(\mathrm{MCKT})$. . . . . . . . . . . . . . 26

5. Block diagram of the 19 -stage CMOS ring oscillator . . . . . . . . . . . . 28

6. Cross-sectional view of an inverter using a twin-tub, $n$-substrate, CMOS bulk process . . . . . . . . . . . . . . . . . . . . . 29

7. Four- $\mu \mathrm{m}$ cross-bridge resistor for determining the polysilicon linewidth . . . . 31

8. $P$-channel MOSFET of the same dimensions as the $p$-channel ring oscillator MOSFETs

9. Plot of the I-V characteristics for a $p$-channel MOSFET on the test chip located on wafer $\mathrm{Y}$ at row 4 column 7 . . . . . . . . . . . . . . . 34

10. Plot of a simulated node potential in the 19-stage CMOS ring oscillator as a function of time for the ring oscillator on the test chip located on wafer $\mathrm{Y}$ at row 5 column 8 


\section{LIST OF TABLES}

1. A Description of the Programs Used in the Computer Procedure KEYS . . . . 6

2. A Brief Description of the Parameters Considered in this Analysis . . . . . . . 9

3. A P-Channel MOSFET Initial Estimates File of SUXES . . . . . . . . . . . 11

4. Methods Used to Obtain Initial Estimates for Input Parameters of SUXES . . 12

5. A P-Channel MOSFET Options File of SUXES . . . . . . . . . . . . . 14

6. A P-Channel MOSFET Strategy File of SUXES . . . . . . . . . . . . 15

7. The Methods Used to Obtain Several Input Parameters for SPICE . . . . . 16

8. A SPICE File to Obtain the I-V Characteristics for a P-Channel MOSFET . . . 17

9. A SPICE File for a 19-Stage CMOS Ring Oscillator . . . . . . . . . . . . 18

10. Example of a File Suitable for Input to STAT2 . . . . . . . . . . . . . . 22

11. Example of a STAT2 Command Sequence Used to Build a Database and Calculate Correlation Coefficients . . . . . . . . . . . . . . . 24

12. A Rank-Ordering of the Magnitudes of the Ten Highest Correlated Parameters with Respect to (a) the Percent Difference Between the Measured and Simulated Ring Oscillator Frequencies, (b) the Measured Frequency, and

(c) the Simulated Frequency . . . . . . . . . . . . . . . . . 25

13. Example of a STAT2 Command Sequence Used to Make a Gray-Tone Map • . 25

14. The Voltage Values Used to Measure the $N$ - and P-Channel MOSFETS . . . 33

15. A Tabular Summary of Each Chip's Results Similar to the Wafer Summary - . 37

C-1 Example of a Data File from the ACCUTEST . . . . . . . . . . . . . . 134

C-2 Example of a SUXES Data File . . . . . . . . . . . . . . . 135

C-3 Example of a Plot File . . . . . . . . . . . . . . . . . . . . . . 136

C-4 The Files Listed in the Order of Their Occurrence in the Computer Procedure KEYS . . . . . . . . . . . . . . . . . . . . . . 137

C-5 The Files to be Edited at the Discretion of the User . . . . . . . . . 139 
Semiconductor Measurement Technology:

EVALUATING A CHIP, WAFER, OR LOT

USING SUXES, SPICE, AND STAT2

\author{
Janet C. Marshall and Richard L. Mattis \\ Semiconductor Electronics Division \\ National Institute of Standards and Technology \\ Gaithersburg, MD 20899
}

\begin{abstract}
The computer procedure KEYS (linKing softwarE to analYze waferS) links SUXES (Stanford University eXtractor of modEl parameterS), SPICE (a Simulation Program with Integrated Circuit Emphasis), and STAT2. Given data points for individual devices, SUXES obtains the model parameters for SPICE. SPICE predicts the behavior of an individual device or an entire circuit. After analyzing each test chip on a wafer, STAT2 determines correlation coefficients and generates wafer maps of selected parameters. These wafer maps are valuable to the designer, modeler, and process engineer.

The entire package accomplishes the following: (1) standardizes the technique of running SUXES and SPICE in an integrated mode; (2) simulates and plots the characteristic curves; (3) simulates and plots the results of an optional dynamic circuit (for example, a ring oscillator); (4) performs steps (2) and (3) for every test chip on each wafer; (5) summarizes the results from each chip, each wafer, and the lot; (6) rank-orders the model parameters for each wafer according to their correlation coefficients with respect to chosen circuit parameters; and (7) generates wafer maps of several quantities. A CMOS 19-stage ring oscillator is used to illustrate the capabilities of KEYS.
\end{abstract}

Key words: circuit simulator; CMOS; ring oscillator; SPICE; STAT2; SUXES; wafer maps 
NOMENCLATURE

\begin{tabular}{lll}
\hline Symbol & Units & Identification \\
\hline$\epsilon_{\mathrm{o}}$ & $\mathrm{F} / \mathrm{m}$ & $\begin{array}{l}\text { Permittivity of free space } \\
8.85 \times 10^{-12} \mathrm{~F} / \mathrm{m}\end{array}$ \\
$\epsilon_{\mathrm{ox}}$ & $\mathrm{F} / \mathrm{m}$ & $\begin{array}{l}\text { Static dielectric constant of } \mathrm{SiO}_{2} \\
3.9 \times \epsilon_{\mathrm{o}}=34.5 \times 10^{-12} \mathrm{~F} / \mathrm{m}\end{array}$ \\
$\epsilon_{\mathrm{s}}$ & $\mathrm{F} / \mathrm{m}$ & $\begin{array}{l}\text { Static dielectric constant of silicon } \\
11.7 \times \epsilon_{\mathrm{o}}=103.5 \times 10^{-12} \mathrm{~F} / \mathrm{m}\end{array}$ \\
$\mathrm{ERR}$ & $\%$ & $\begin{array}{l}\text { The average error is the sum of the absolute } \\
\text { value of the normal errors divided by the }\end{array}$ \\
& number of points. The normal error is the \\
percent difference between the measured data \\
points and SUXES's predicted data points.
\end{tabular}




\section{INTRODUCTION}

This report describes a computer procedure called KEYS (linKing softwarE to analYze waferS) that was developed at the National Institute of Standards and Technology. KEYS links SUXES (Stanford University eXtractor of modEl parameterS) [1], ${ }^{*}$ SPICE (a Simulation Program with Integrated Circuit Emphasis) [2], and STAT2 [3] to facilitate integrated circuit evaluation. Other examples of packages for integrated circuit evaluation have been described $[4,5]$. A simplified block diagram for KEYS is given in figure 1 .

SPICE predicts currents and voltages at chosen nodes in a circuit and requires a models file which contains input parameters for the devices in the circuit. The accuracy of these input parameters and the device models determines how well the simulated currents and voltages predict the measured currents and voltages. Since developing a models file for SPICE is a difficult task, a computer program called SUXES was developed. SUXES obtains most of these model parameters and adapts to any model. Given initial estimates for the parameter values, upper and lower bounds for these values, and measured data points for each device, SUXES optimizes the parameter values by varying them slightly, according to an initial estimates file, an options file, and a strategy file, so as to decrease the root mean square (rms) deviation between the predicted and measured data points. To aid process engineers, modelers, and designers, STAT2 determines correlation coefficients with respect to selected parameters and generates wafer maps of selected parameters. These maps provide a visual inspection of the positional variations of parameter values over a wafer.

The flowchart for the computer procedure KEYS is given in figure 2. To perform these tasks, additional FORTRAN programs as given in Appendix A were written. Command files connect these programs so that they may run as a single entity. The software used in KEYS is listed in table 1.

In the computer procedure KEYS, there are three sets of parameters: (1) the estimated input parameters for SUXES, (2) SUXES's optimized parameters, and (3) the model input parameters for SPICE. Given the data points for individual devices, SUXES obtains the model parameters for SPICE. The combination of SUXES and SPICE gives a procedure for parameterizing experimental data from circuits. SUXES's optimized parameters may not be physically meaningful. Nonetheless, in this report, the assumption is made that SUXES's optimized parameters have the same definition as the model input parameters for SPICE. Hence, the software to be presented directly inserts these parameters into the models file of SPICE.

The computer procedure KEYS characterizes a chip, wafer, or lot. In this report, a 19-stage

* Certain commercial equipment, instruments, and computer programs are identified in this paper in order to specify the experimental procedure adequately. Such identification does not imply recommendation or endorsement by the National Institute of Standards and Technology, nor does it imply that the equipment or program identified are necessarily the best available for the purpose. 


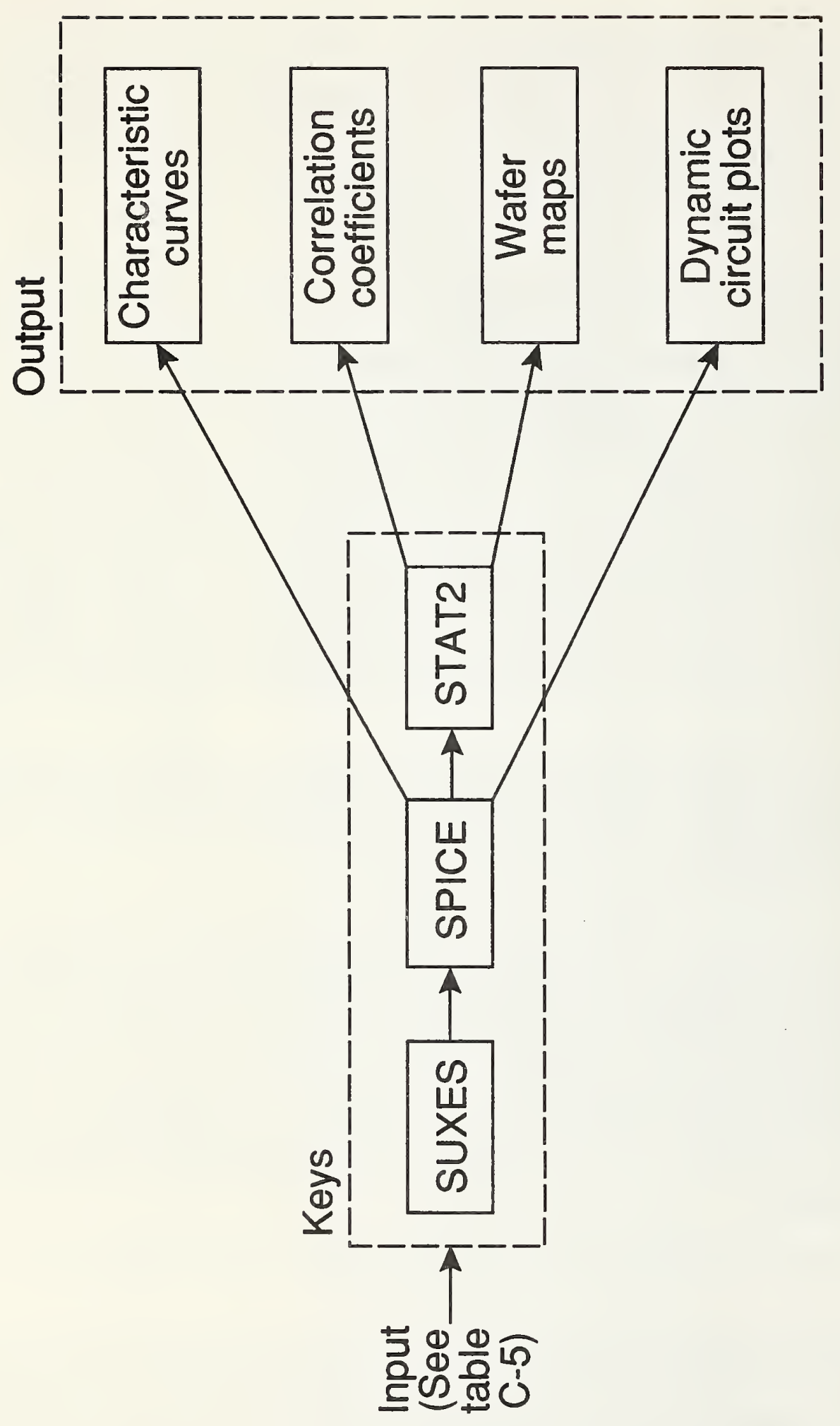

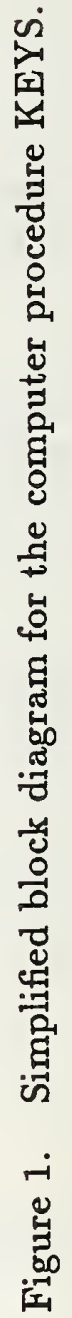




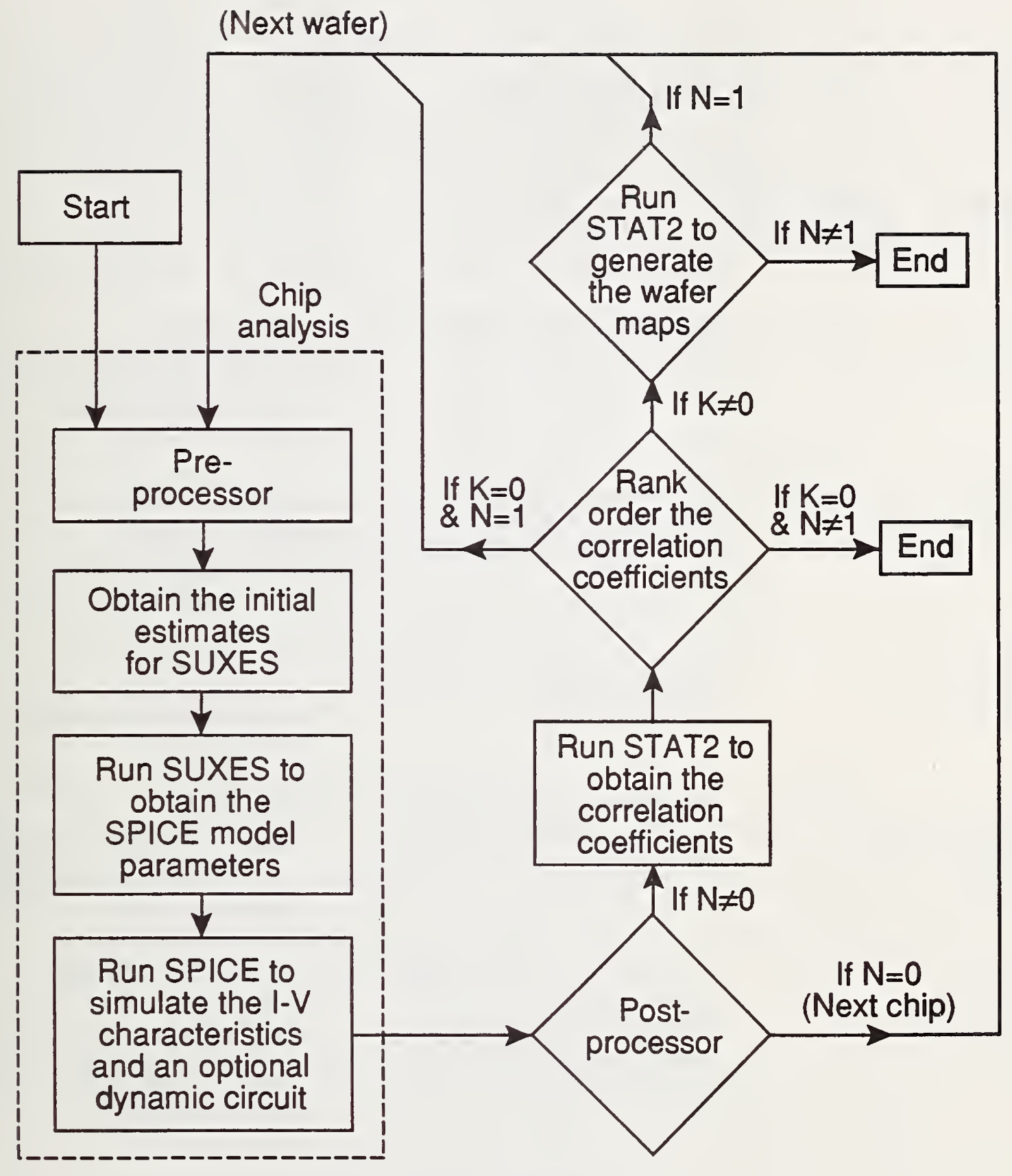

Figure 2. Flowchart for the computer procedure KEYS. 
Table 1. A Description of the Programs Used in the Computer Procedure KEYS

\begin{tabular}{|c|c|c|c|}
\hline \begin{tabular}{ll} 
& \multicolumn{2}{l}{${ }^{\#}$} \\
PROGRAM & ST
\end{tabular} & $\begin{array}{l}\text { OF SOL } \\
\text { PROGR } \\
\text { TATEMF }\end{array}$ & PURPOSE & AUTHOR \\
\hline $\begin{array}{l}\text { SUXES } \\
(\text { version I) }\end{array}$ & 3792 & $\begin{array}{l}\text { Obtains the model parameters } \\
\text { for SPICE }\end{array}$ & $\begin{array}{l}\text { K. Doganis } \\
\text { Electrical Engineering } \\
\quad \text { Software } \\
\text { Santa Clara, Calif. } \\
\text { (408) 296-8151 }\end{array}$ \\
\hline $\begin{array}{l}\text { SPICE } \\
\quad \text { (version 2G.5) }\end{array}$ & 43414 & $\begin{array}{l}\text { The circuit simulator which } \\
\text { obtains the I-V characteristics } \\
\text { and simulates a dynamic circuit }\end{array}$ & $\begin{array}{l}\text { Univ. of Calif. } \\
\text { Berkeley, Calif. } \\
\text { with revisions by } \\
\text { R. Mattis } \\
\text { (301) } 975-2235\end{array}$ \\
\hline $\begin{array}{l}\text { NCAR Graphics } \\
\text { MCVAX } \\
\text { UTILITY }\end{array}$ & s 1764 & $\begin{array}{l}\text { Makes plots on the specified } \\
\text { output devices }\end{array}$ & $\begin{array}{l}\text { NCAR Graphics } \\
\text { Information } \\
\text { NCAR, Boulder, Colo. } \\
\text { (303) 497-1201 }\end{array}$ \\
\hline $\begin{array}{l}\text { STAT2 } \\
\text { (version 1.31) } \\
\text { CRDB } \\
\text { CMLIB }\end{array}$ & 16212 & $\begin{array}{l}\text { Determines the correlation } \\
\text { coefficients and generates } \\
\text { wafer maps }\end{array}$ & $\begin{array}{l}\text { R. Mattis } \\
\text { Electronics and Electrical } \\
\text { Engineering Laboratory } \\
\text { NIST, Gaithersburg, Md. } \\
\text { (301) } 975-2235\end{array}$ \\
\hline $\begin{array}{l}\text { Misc. Files } \\
\text { (version I) }\end{array}$ & 1620 & $\begin{array}{l}\text { These include the command } \\
\text { procedures, the editor files, } \\
\text { the data files, and the } \\
\text { FORTRAN files needed to } \\
\text { link the software }\end{array}$ & $\begin{array}{l}\text { J. Marshall, R. Mattis, } \\
\text { and M. Gaitan } \\
\text { Electronics and Electrical } \\
\text { Engineering Laboratory } \\
\text { NIST, Gaithersburg, Md. } \\
\text { (301) } 975-2052\end{array}$ \\
\hline
\end{tabular}


ring oscillator is used to illustrate the procedure. This example takes approximately 11 CPU minutes per chip on a VAX-11/780. The entire package accomplishes the following: (1) standardizes the technique of running SUXES and SPICE in an integrated mode; (2) simulates and plots the characteristic curves; (3) simulates and plots the waveforms of an optional dynamic circuit (for example, a ring oscillator); (4) performs steps (2) and (3) for every test chip on each wafer if desired; (5) summarizes the results from each chip, each wafer, and the lot; (6) rank-orders the model parameters for each wafer according to their correlation coefficients with respect to chosen circuit parameters; and (7) generates wafer maps of selected parameters. The wafer maps are typically made for the most highly correlated parameters. Therefore, a careful selection of the chosen parameters with regard to the correlation coefficients will improve the usefulness of the wafer maps to process engineers, modelers, and designers.

This report contains three parts: Motivation and General Principles, An Example Using CMOS Technology, and the Conclusions. In the first part, Section 2.1 describes the linking of SUXES, SPICE, and STAT2. It is assumed that the reader is familiar with SUXES, SPICE, and STAT2, so these programs are only briefly described in sections $2.2,2.3$, and 2.4. In the second part, section 3.1 describes the test structure measurement methods, and section 3.2 discusses the output which consists of plots, tabular listings, and wafer maps. Appendix A contains the listing of the computer procedure KEYS. Appendix B presents a method of modifying SUXES's strategy file to decrease the rms deviation between the measured data points and SUXES's predicted data points while still providing realistic curves. Finally, Appendix C describes the procedure to run KEYS.

\section{MOTIVATION AND GENERAL PRINCIPLES}

\subsection{Linking SUXES, SPICE, and STAT2}

SUXES, SPICE, and STAT2 are the programs described in the following sections. SUXES supplies model parameters for SPICE, SPICE simulates circuits, and STAT2 obtains correlation coefficients and generates wafer maps. KEYS can be used to provide a smooth transition between these programs. The following three paragraphs demonstrate this and the last paragraph describes how it is done.

Typically, the estimated input parameters for SUXES are manually inserted into SUXES's initial estimates file. The estimated input parameters vary, for example, with oxide thickness. Hence, these values need to be calculated for each value of oxide thickness. KEYS can be used to perform these calculations and eliminate manual intervention.

Typically, the model input parameters for SPICE are manually inserted into the models file of SPICE. This becomes a task which is burdensome since each chip has its own set of parameters. KEYS can be used to insert directly SUXES's optimized parameters into the models file of SPICE. The values of the model input parameters for SPICE are also stored for future correlation coefficient analyses and possible wafer maps.

Typically, the values for the model input parameters for SPICE are manually inserted into 
files suitable for STAT2. KEYS can be used to place these values into the appropriate files to eliminate manual intervention. Thus, analyzing chips, wafers, and lots has become an easy task.

In smoothing the transitions between SUXES, SPICE, and STAT2, KEYS uses the EDT editor (copyright, Digital Equipment Corporation). Some editors can be imbedded in automated programs and can actually edit a file while the program is executing. EDT is an example of such an editor. This eliminates human intervention and error. A command procedure calls the file to be edited, and uses the editor commands in a specified file. Thus, it places the parameter values from SUXES into SPICE, and it modifies command procedures to reference the data files for the next chip. The linking of the three software packages is accomplished in this manner. The procedure to run KEYS is given in Appendix C.

\subsection{SUXES}

SUXES [1] obtains the model parameters for SPICE $[2,6,7]$. A model-independent Levenberg-Marquardt algorithm is used to minimize the rms deviation between the measured and predicted data points. This minimization makes it possible to perform a SPICE analysis more accurately. Given initial estimates for the parameter values, upper and lower bounds for these values, and measured data points for each device, the parameter values are optimized by varying them slightly, according to an initial estimates file, an options file, and a strategy file, so as to decrease the rms deviation between the predicted and measured data points. In the computer procedure KEYS, the parameters optimized by SUXES are indicated in table 2.

In KEYS the following input files are needed to run SUXES: the data file(s), the initial estimates file, the options file, and the strategy file. After the optimization, an extracted parameters file and a plot file are created. The extracted parameters file contains the optimized values of the parameters, and the plot file contains the predicted data points.

The data file(s) contain(s) the measured data points. The proper polarities are used for the devices. There is an exception: for $p$-channel MOSFETs, a positive current must be recorded, or the device must be treated as an $n$-channel MOSFET. The automated procedure ensures that the proper polarities are used in SUXES.

The initial estimates file includes the initial guesses for the parameter values, the lower bounds, and the upper bounds for these parameters. The parameter values are free to vary within these specified boundaries. The upper and lower bounds used in this analysis were obtained using the technique described in the SUXES report [1] or from the method described in Appendix B to find an appropriate strategy file. No attempt was made to quantify the values of these bounds (say $\pm 10 \%$ or $\pm \sigma$ about the initial estimate), since each parameter has its own optimization sensitivity. The user may alter these values to increase the number of acceptable chips. In KEYS, an acceptable chip is one whose optimized SUXES values are not pegged at the lower or upper bounds. In the example with CMOS technology, there are two initial estimates files: NINPUT.DAT is for the $n$-channel 
Table 2. A Brief Description of the Parameters Considered in this Analysis

\section{PARAMETER UNITS PARAMETER DESCRIPTION}

\begin{tabular}{|c|c|c|}
\hline LEVEL & - & model index \\
\hline TYPE & - & $\begin{array}{l}\text { type of device: } \\
+1 \quad n \text {-channel device } \\
-1 \quad p \text {-channel device }\end{array}$ \\
\hline $\begin{array}{l}* \mathrm{LD} \\
* \mathrm{TOX}\end{array}$ & & $\begin{array}{l}\text { lateral diffusion } \\
\text { oxide thickness }\end{array}$ \\
\hline * NSUB & $\mathrm{cm}^{-3}$ & substrate doping \\
\hline *VTO & V & zero-bias threshold voltage \\
\hline * KP & $\mathrm{A} / \mathrm{V}^{2}$ & intrinsic transconductance parameter \\
\hline * GAMMA & $\mathrm{V}^{0.5}$ & bulk threshold parameter \\
\hline$*$ PHI & $\mathrm{V}$ & surface potential at strong inversion \\
\hline *UO & $\mathrm{cm}^{2} / \mathrm{V}-\mathrm{s}$ & surface mobility at low gate voltages \\
\hline * DELTA & - & width effect on threshold voltage \\
\hline *VMAX & $\mathrm{m} / \mathrm{s}$ & maximum drift velocity of carriers \\
\hline *XJ & & metallurgical junction depth \\
\hline $\begin{array}{l}* \text { KAPPA } \\
\text { * NFS }\end{array}$ & $\mathrm{cm}^{-2}$ & $\begin{array}{l}\text { saturation tield factor } \\
\text { effective fast surface state density }\end{array}$ \\
\hline *ETA & - & static feedback effect parameter \\
\hline * THETA & $\mathrm{V}^{-1}$ & empirical mobility modulation parameter \\
\hline CJSW & $\mathrm{F} / \mathrm{m}$ & $\begin{array}{l}\text { zero-bias bulk junction sidewall capacitance per meter } \\
\text { of junction perimeter }\end{array}$ \\
\hline CGDO & $\mathrm{F} / \mathrm{m}$ & gate-drain overlap capacitance per meter channel width \\
\hline CGSO & $\mathrm{F} / \mathrm{m}$ & gate-source overlap capacitance per meter channel width \\
\hline CGBO & $\mathrm{F} / \mathrm{m}$ & gate-bulk overlap capacitance per meter channel length \\
\hline RSH & $\Omega / \square$ & sheet resistance of the drain and source diffusions \\
\hline JS & $\mathrm{A} / \mathrm{m}^{2}$ & $\begin{array}{l}\text { bulk junction saturation current per square meter of } \\
\text { junction area }\end{array}$ \\
\hline TPG & - & $\begin{array}{l}\text { type of gate material: } \\
\begin{aligned}+1 & \text { opposite to substrate } \\
-1 & \text { same as substrate } \\
0 & \text { Al gate }\end{aligned}\end{array}$ \\
\hline MJ & - & bulk junction bottom grading coefficient \\
\hline MJSW & - & bulk junction sidewall grading coefficient \\
\hline PB & V & bulk junction potential \\
\hline XQC & - & $\begin{array}{l}\text { thin-oxide capacitance model flag and coefficient of } \\
\text { channel charge share attributed to drain }(0.0-0.5)\end{array}$ \\
\hline LAMBDA & $\mathrm{V}^{-1}$ & channel length modulation parameter \\
\hline UEXP & - & critical field exponent \\
\hline UCRIT & $\mathrm{V} / \mathrm{m}$ & critical field for mobility degradation \\
\hline NEFF & & total channel charge coefficient \\
\hline NSS & $\mathrm{cm}^{-}$ & effective surface charge density \\
\hline
\end{tabular}

* The parameters found via SUXES, in this analysis. 
MOSFETs, and PINPUT.DAT shown in table 3 is for the $p$-channel MOSFETs.

This list contains all the parameters that SUXES can optimize. In this analysis a subset of these parameters is used. The methods used to obtain the initial estimates for this subset of parameters are given in table 4 . The other parameter values are initialized to the default values specified in this table.

The options file selects the desired data points from the data file(s), determines any weighting of the data, and determines the method of convergence or termination. In the example with CMOS technology, all the data points are used in SUXES. The options file for the $n$-channel MOSFETs is called NOPT.FIL, and the options file for the $p$-channel MOSFETs, shown in table 5, is called POPT.FIL. This table also provides the definitions of the convergence/termination criteria used in this file. Additional information can be found in the original report on SUXES [1].

The strategy file selects the order and groups of parameter values to be optimized. In the example with CMOS technology, the parameters whose values are optimized are: LD, TOX, NSUB, VTO, KP, GAMMA, PHI, UO, DELTA, VMAX, XJ, KAPPA, NFS, ETA, and THETA. Refer to table 2 for the parameter descriptions. The strategy file for the $n$-channel MOSFETs is called NSTRAT.FIL, and the strategy file for the $p$-channel MOSFETs, shown in table 6 , is called PSTRAT.FIL. A parameter's value is optimized if a " 1 " appears in the column corresponding to the iteration of interest. A procedure is described in Appendix B which modifies the strategy file in order to decrease the rms deviation between the measured data points and SUXES's predicted data points. This procedure provides curves that are consistent with the measured data.

After the optimization is complete, SUXES generates a plot file. In the example with CMOS technology, the $I_{d s}$ values are predicted for all combinations of $V_{g s}$ and $V_{d s}$ values. Therefore, if the voltages are measured, a tremendous number of I-V curves are generated. To save memory, to decrease the computer computation time, and to have a plot file of a manageable size, the measured voltage values in the data file(s) should be rounded or truncated before running SUXES. The automated procedure does this.

The capacitive parameters (CGDO, CGSO, and CGBO) are calculated after the optimization. The equations given in table 7 are used with the appropriate values from the optimization.

\subsection{SPICE}

SPICE performs dc, transient, or ac small-signal analyses of circuits $[2,6,7]$. Dynamic circuits are typically simulated using a transient analysis which predicts branch currents and node voltages as a function of time. A dc analysis determines the circuit's operating point, making it possible to obtain the characteristic curves. The ac small-signal analysis determines the ac variables as a function of frequency. In the example given, the I-V characteristics for the $p$-channel MOSFETs and the response of the optional dynamic circuit (the ring oscillator) were simulated using the SPICE files listed in tables 8 and 9 , respectively. The files used to get the I-V characteristics for the $n$-channel MOSFETs are 
Table 3. A P-Channel MOSFET Initial Estimates File of SUXES

\begin{tabular}{llccc}
$*$ & FILENAME: & PINPUT.DAT & & \\
$*$ & & & \\
$*$ & parameters & initial & lowerbound & upperbound \\
$*$ & & 1 & 3 \\
LEVEL & 3 & -1.0 & 1.0 \\
TYPE & -1.0 & 0.1 & 2.0 \\
LD & 0.45 & 250.0 & 1000.0 \\
TOX & 635.0 & $1.0 \times 10^{13}$ & $1.0 \times 10^{17}$ \\
NSUB & $1.2 \times 10^{16}$ & -3.0 & -0.1 \\
VTO & -0.85 & $1.0 \times 10^{-6}$ & $100 \times 10^{-6}$ \\
KP & $14.02 \times 10^{-6}$ & 0.1 & 2.0 \\
GAMMA & 1.16 & 0.3 & 12.9 \\
PHI & 0.3618 & 0.1 & 2.3 \\
UO & 500.0 & 0.001 & $1.0 \times 10^{6}$ \\
UEXP & 0.0 & $1.0 \times 10^{4}$ & $1.0 \times 10^{2}$ \\
UCRIT & 0.0 & $1.0 \times 10^{2}$ & $9.9 \times 10^{7}$ \\
DELTA & 0.6749 & 0.1 & 1.5 \\
VMAX & $3.24 \times 10^{5}$ & $1.0 \times 10^{-6}$ & $1.0 \times 10^{-1}$ \\
XJ & 0.66 & 0.0 & 999.0 \\
LAMBDA & 0.0 & $1.0 \times 10^{10}$ & $4.0 \times 10^{14}$ \\
KAPPA & 2.0 & $1.0 \times 10^{-2}$ & $1.0 \times 10^{1}$ \\
NFS & $1.17 \times 10^{12}$ & 0.0 & $1.0 \times 10^{14}$ \\
NEFF & 0.0 & -1.0 & 1.0 \\
NSS & $1.0 \times 10^{11}$ & -10.0 & 10.0 \\
TPG & -1.0 & -10.0 & 10.0 \\
ETA & 0.1210 & & \\
THETA & 0.2396 & & \\
\hline
\end{tabular}


Table 4. Methods Used to Obtain Initial Estimates for Input Parameters of SUXES

\section{PARAMETER DEFAULT VALUE USED OR HOW OBTAINED}

\begin{tabular}{ll}
\hline LEVEL & Default value $=3$ \\
TYPE & $N$-channel MOSFET default value $=1.0$ \\
& $P$-channel MOSFET default value $=-1.0$ \\
LD & $N$-channel MOSFET default value $=0.38 \mu \mathrm{m}$ \\
& $P$-channel MOSFET default value $=0.45 \mu \mathrm{m}$
\end{tabular}

TOX Default value $=63.5 \mathrm{~nm}$

NSUB $\quad$ NSUB $=\left(\frac{1}{2\left(\epsilon_{\mathrm{s}}\right) q}\right)\left[\frac{\operatorname{GAMMA}\left(\epsilon_{o x}\right)}{\operatorname{TOX}}\right]^{2}$

VTO (1) This is measured at $\mathrm{V}_{\mathrm{ds}}=0.2 \mathrm{~V}$ and $\mathrm{V}_{\mathrm{bs}}=0.0 \mathrm{~V}$ for the $n$-channel devices.

(2) This is measured at $V_{d s}=-0.2 \mathrm{~V}$ and $V_{b s}=0.0 \mathrm{~V}$ for the $p$-channel devices.

(3) A five-point maximum slope technique is used.

(4) When the maximum slope is found, a straight line is extrapolated to the $\mathrm{x}$-axis and $0.5 \mathrm{~V}_{\mathrm{ds}}$ is subtracted from the $\mathrm{V}_{\mathrm{gs}}$ value to obtain the threshold voltage.

(5) $\mathrm{VTO}=$ the threshold voltage

KP

(1) This is measured at $V_{d s}=0.2 \mathrm{~V}$ and $V_{b s}=0.0 \mathrm{~V}$ for the $n$-channel devices.

(2) This is measured at $V_{d s}=-0.2 \mathrm{~V}$ and $V_{b s}=0.0 \mathrm{~V}$ for the $p$-channel devices.

(3) $\mathrm{KP}=\frac{\operatorname{maxslope}(\mathrm{L})}{\mathrm{V}_{\mathrm{ds}}(\mathrm{W})}$

(4) An unreasonable value of KP causes the computer program to abort.

GAMMA (1) GAMMA = |slope $\mid$ of the threshold voltage vs.

$\left[\sqrt{\left|\mathrm{V}_{\mathrm{bs}}\right|+\text { tphif }}-\sqrt{\mathrm{tphif}}\right]$

(2) Assume tphif $=0.6 \mathrm{~V}$.

(3) For the $n$-channel MOSFETs, measurements are taken at $\mathrm{V}_{\mathrm{bs}}=\mathbf{- 3 . 0}$, -6.0 , and $-9.0 \mathrm{~V}$ for $\mathrm{V}_{\mathrm{ds}}=0.2 \mathrm{~V}$.

(4) For the $p$-channel MOSFETs, measurements are taken at $\mathrm{V}_{\mathrm{bs}}=3.0$, 6.0 , and $9.0 \mathrm{~V}$ for $\mathrm{V}_{\mathrm{ds}}=-0.2 \mathrm{~V}$.

(5) A five-point maximum slope technique is used. When the maximum slope is found, a straight line is extrapolated to the $x$-axis and $0.5 V_{d s}$ is subtracted from the $\mathrm{V}_{\mathrm{gs}}$ value to obtain the threshold voltage.

(6) A recorded default value for a measured threshold voltage causes the computer program to abort.

PHI

$\mathrm{PHI}=2\left(\frac{\mathrm{kT}}{\mathrm{q}}\right) \ln \left(\frac{\mathrm{NSUB}}{\mathrm{n}_{\mathrm{i}}}\right)$ where $\mathrm{n}_{\mathrm{i}}=1.45 \times 10^{10} \mathrm{~cm}^{-3}$ at $300 \mathrm{~K}$

The simulated ring oscillator frequencies vary by less

than $0.2 \%$ for values of $n_{i}$ between $1.00 \times 10^{10} \mathrm{~cm}^{-3}$ and $1.45 \times 10^{10} \mathrm{~cm}^{-3}$. 
UO

UEXP

UCRIT

DELTA

VMAX

$\mathrm{XJ}$

LAMBDA

KAPPA

NFS

NEFF

NSS

TPG

ETA

THETA

$\mathrm{UO}=\frac{\mathrm{KP}(\mathrm{TOX})}{\epsilon_{\mathrm{ox}}}$

This is not a MOS3 SPICE input parameter.

Default value $=0.0$

This is not a MOS3 SPICE input parameter.

Default value $=0.0$

The value of this parameter is preset to be the value it typically is from previous SUXES runs.

$N$-channel MOSFET default value $=2.685$

$P$-channel MOSFET default value $=0.6749$

The value of this parameter is preset to be the value it typically is from previous SUXES runs.

$N$-channel MOSFET default value $=7.9 \times 10^{5} \mathrm{~m} / \mathrm{s}$

$P$-channel MOSFET default value $=3.24 \times 10^{5} \mathrm{~m} / \mathrm{s}$

$\mathrm{XJ}=\frac{\mathrm{LD}}{0.8}$

This is not a MOS3 SPICE input parameter.

Default value $=0.0$

The value of this parameter is preset to be the value that helps the theory match the experiment.

$N$-channel MOSFET default value $=0.80$

$P$-channel MOSFET default value $=0.20$

The value of this parameter is preset to be the value it typically is from previous SUXES runs.

$N$-channel MOSFET default value $=2.28 \times 10^{12} \mathrm{~cm}^{-2}$

$P$-channel MOSFET default value $=1.17 \times 10^{12} \mathrm{~cm}^{-2}$

This is not a MOS3 SPICE input parameter.

Default value $=0.0$

Default value $=10^{11} \mathrm{~cm}^{-2}$

$N$-channel MOSFET default value $=1.0$

$P$-channel MOSFET default value $=-1.0$

The value of this parameter is preset to be the value it typically is from previous SUXES runs.

$N$-channel MOSFET default value $=0.1306$

$P$-channel MOSFET default value $=0.1210$

The value of this parameter is preset to be the value it typically is from previous SUXES runs.

$N$-channel MOSFET default value $=0.3612 \mathrm{~V}^{-1}$

$P$-channel MOSFET default value $=0.2396 \mathrm{~V}^{-1}$ 
Table 5. A P-Channel MOSFET Options File of SUXES

$*$

* FILENAME: POPT.FIL

*

* $\quad$ Nsign $=$ Number of significant digits

* Maxfn = Maximum number of function evaluations

* $\quad$ Delta $=$ Norm of gradient bound

* $\quad$ Epsil = Sum of squares difference

* Linit = Marquardt parameter initial value

* $\quad$ Lscal = Marquardt parameter scale

* Luppr = Marquardt parameter upper value

$* \quad F c d s w=$ Forward $/$ central difference switch

*

Criteria

Nsign=4 Maxfn=200 Delta $=1.0 \times 10^{-9}$ Epsil $=1.0 \times 10^{-6}$

Linit $=0.1 \mathrm{Lscal}=2.0 \mathrm{Luppr}=1000 \mathrm{Fcdsw}=0.1$

*

RangVd $\min =-5.5 \quad \max =0.00 \quad$ incr $=0.0$

* RangVg $\min =-5.5 \quad \max =0.00 \quad$ incr $=0.0$

*

RangVb $\min =0.0 \quad \max =0.0 \quad$ incr $=1.0$

*

* Weight

* 
Table 6. A P-Channel MOSFET Strategy File of SUXES

\begin{tabular}{|c|c|c|c|c|c|}
\hline \multirow{3}{*}{$\begin{array}{ll}* & \text { FILENAME: } \\
* & \\
* & \\
* & \text { parameters } \\
* & \end{array}$} & \multicolumn{2}{|c|}{ PSTRAT.FIL } & \multirow{3}{*}{$\begin{array}{c}\text { third } \\
\text { iteration }\end{array}$} & \multirow{3}{*}{$\begin{array}{c}\text { fourth } \\
\text { iteration }\end{array}$} & \multirow{3}{*}{$\begin{array}{c}\text { fifth } \\
\text { iteration }\end{array}$} \\
\hline & first & second & & & \\
\hline & iteration & iteration & & & \\
\hline LEVEL & 0 & 0 & 0 & 0 & 0 \\
\hline TYPE & 0 & 0 & 0 & 0 & 0 \\
\hline $\mathrm{LD}$ & 0 & 0 & 1 & 0 & 0 \\
\hline TOX & 0 & 1 & 0 & 0 & 1 \\
\hline NSUB & 0 & 0 & 1 & 0 & 0 \\
\hline VTO & 0 & 1 & 0 & 0 & 1 \\
\hline $\mathrm{KP}$ & 0 & 0 & 1 & 0 & 0 \\
\hline GAMMA & 0 & 1 & 0 & 0 & 1 \\
\hline PHI & 0 & 0 & 0 & 1 & 0 \\
\hline $\mathrm{UO}$ & 0 & 0 & 0 & 1 & 0 \\
\hline UEXP & 0 & 0 & 0 & 0 & 0 \\
\hline UCRIT & 0 & 0 & 0 & 0 & 0 \\
\hline DELTA & 1 & 1 & 0 & 0 & 1 \\
\hline VMAX & 0 & 0 & 0 & 1 & 0 \\
\hline $\mathrm{XJ}$ & 0 & 0 & 1 & 0 & 0 \\
\hline LAMBDA & 0 & 0 & 0 & 0 & 0 \\
\hline KAPPA & 0 & 0 & 0 & 1 & 0 \\
\hline NFS & 0 & 1 & 0 & 0 & 1 \\
\hline NEFF & 0 & 0 & 0 & 0 & 0 \\
\hline NSS & 0 & 0 & 0 & 0 & 0 \\
\hline TPG & 0 & 0 & 0 & 0 & 0 \\
\hline ETA & 1 & 0 & 0 & 0 & 0 \\
\hline THETA & 1 & 0 & 0 & 0 & 0 \\
\hline
\end{tabular}


Table 7. The Methods Used to Obtain Several Input Parameters for SPICE

CJSW

CGDO

CGSO

CGBO

RSH

JS

MJ

MJSW

PB

XQC
Default value $=0.6 \times 10^{-9} \mathrm{~F} / \mathrm{m}$

CGDO $=\frac{\epsilon_{\text {ox }} L D}{\text { TOX }}$

where LD is the lateral diffusion

$\mathrm{CGSO}=\mathrm{CGDO}$

$\mathrm{CGBO}=\frac{\epsilon_{\mathrm{OX}} \mathrm{GO}}{2 \mathrm{TOX}}$

where GO is the design rule of the gate overlap $=4 \mu \mathrm{m}$

$N$-channel MOSFET default value $=11.63 \Omega / \square$ $P$-channel MOSFET default value $=61.57 \Omega / \square$

Default value $=5.0 \times 10^{-5} \mathrm{~A} / \mathrm{m}^{2}$

Default value $=0.5$

Default value $=0.5$

Default value $=0.9 \mathrm{~V}$

Default value $=0.45$ 
Table 8. A SPICE File to Obtain the I-V Characteristics for a P-Channel MOSFET

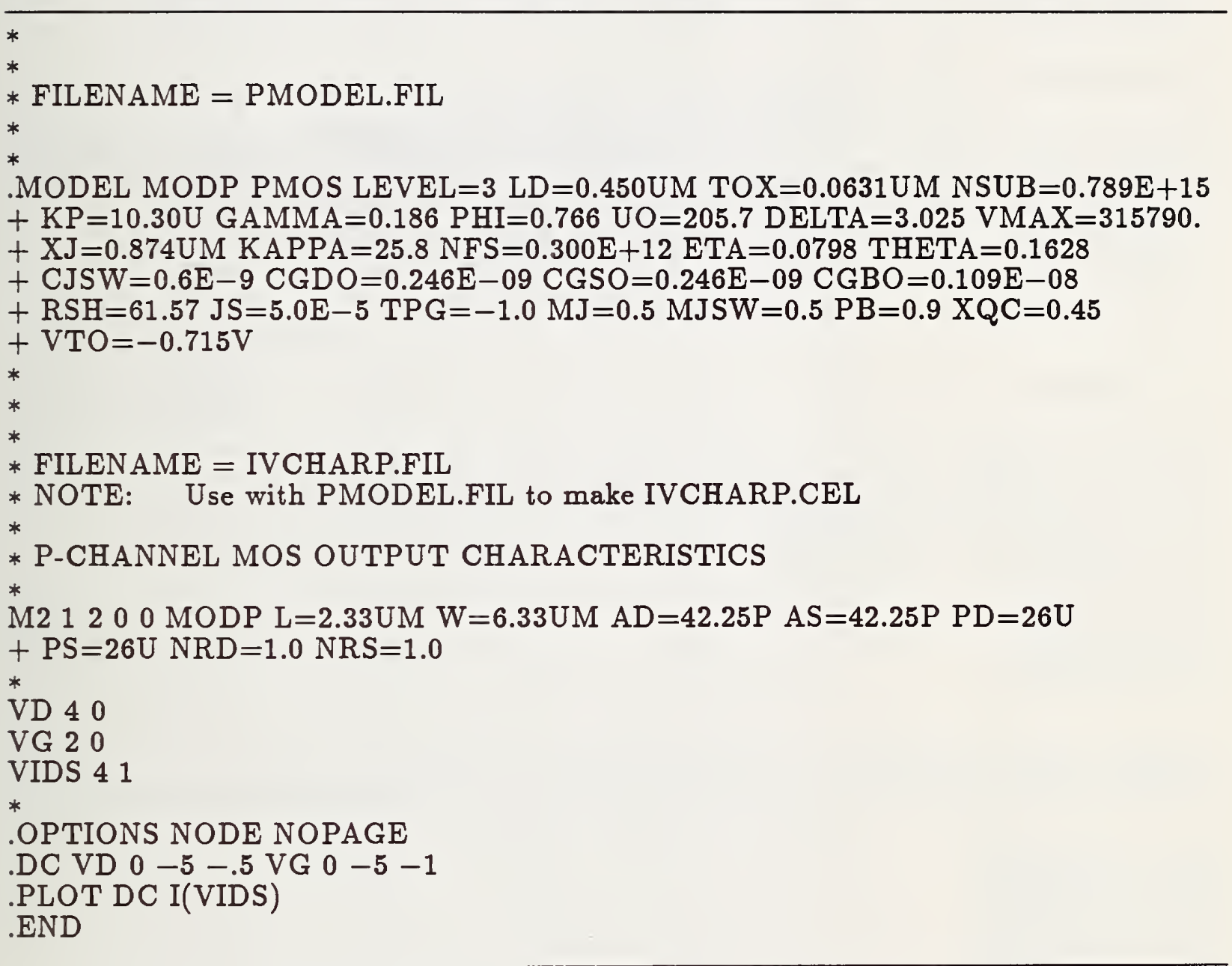


Table 9. A SPICE File for a 19-Stage CMOS Ring Oscillator

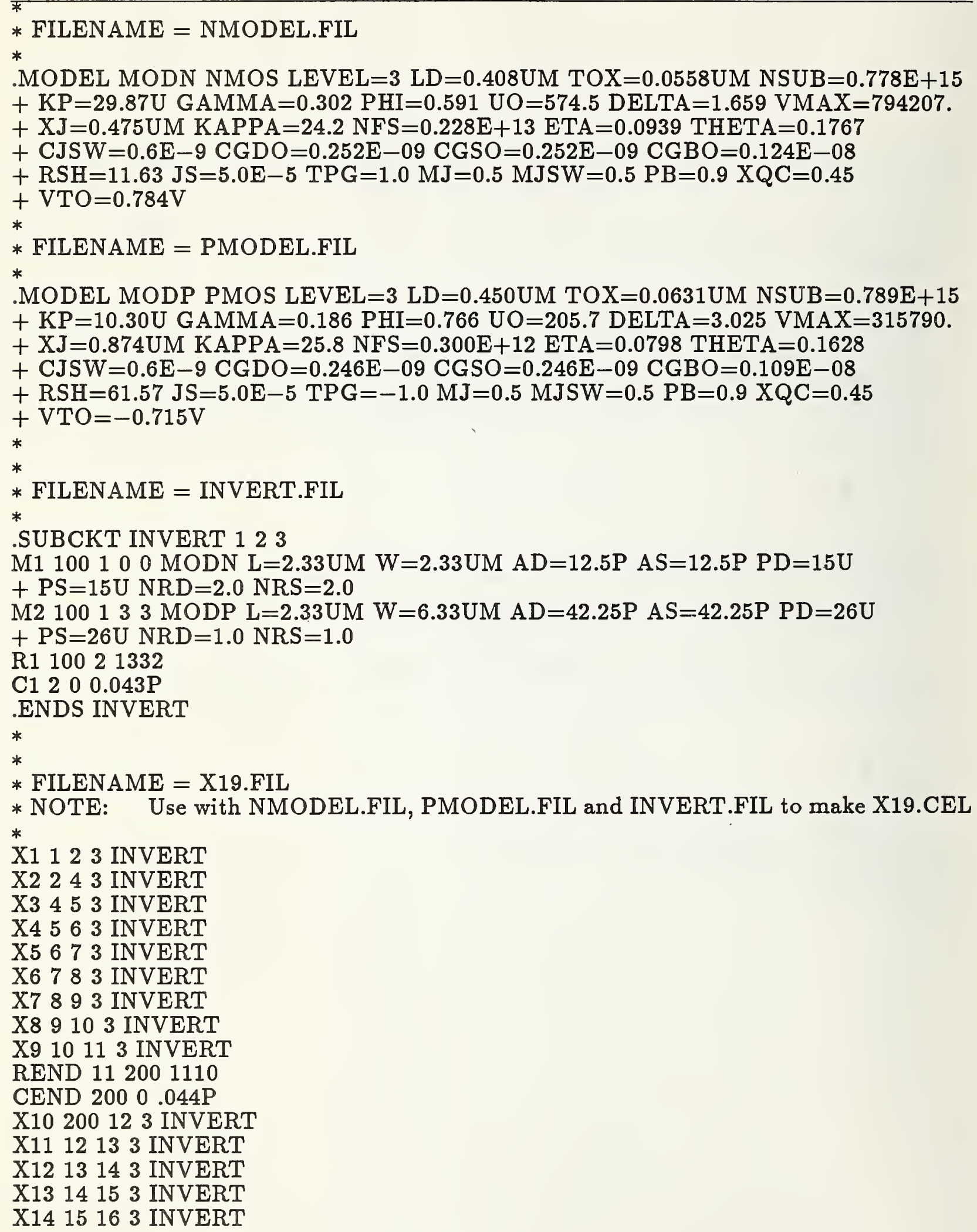




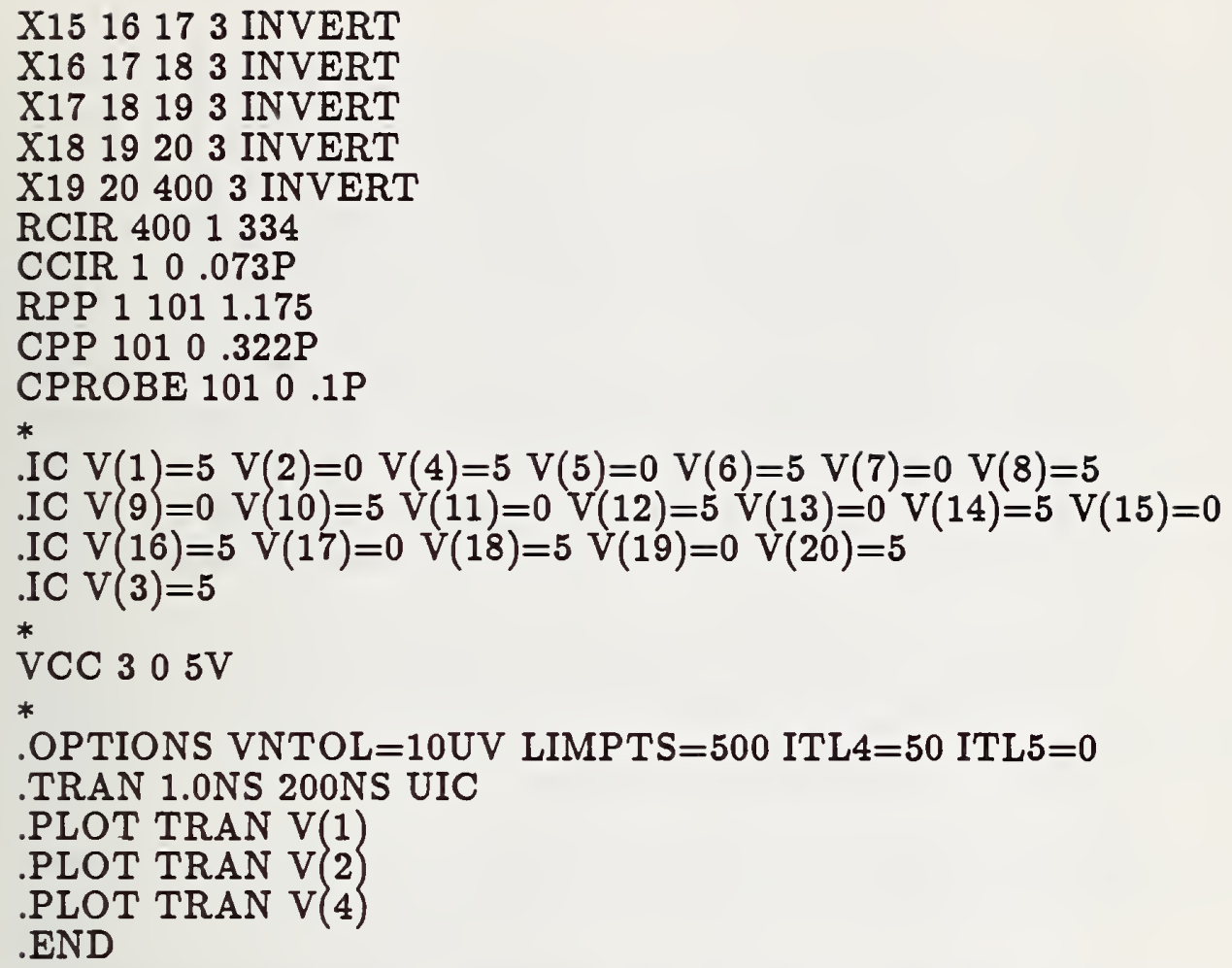


similar to those of the $p$-channel MOSFETs. MOS3 was the model used in SPICE 2G.5 for all the simulations.

To perform the simulations, a models file is needed to characterize the devices. It is assumed that SUXES's optimized parameters have the same definition as the input model parameters for SPICE. Hence, these parameters are directly inserted into the models file of SPICE. SUXES supplies most of the model parameter values. Several additional parameters (CGDO, CGSO, and CGBO) are calculated solely from the optimized SUXES values according to the equations given in table 7 . In the computer procedure KEYS, the parameters LEVEL and TYPE are predetermined (as specified in table 4) and not allowed to be changed by SUXES. Several parameters are used in SPICE but cannot be optimized with the current version of SUXES. They are: CJSW, RSH, JS, MJ, MJSW, PB, and XQC. These parameters are considered noncritical [8]. The parameters mentioned above are briefly described in table 2 , and the default values used in this analysis are listed in table 7 .

In addition to the models file, SPICE requires that the user supply the circuit elements and connections, any voltage or current sources, the initial conditions, the type of analysis, the output desired, and other pertinent SPICE information.

To obtain realistic simulations, especially as device dimensions are reduced, it becomes necessary to include parasitic elements in the simulations. The parasitic elements included in the example given in this report are the source and drain diffusion resistances, the polysilicon resistances and capacitances, the metal resistances and capacitances, and the probe capacitance.

The two most common errors that occur when simulating a circuit are the inability of the values of the node potentials in the circuit to converge and the internal timestep being too small in the transient analysis. To avoid these two problems, the following can be done:

(1) Initialize each node in the circuit to an appropriate starting potential.

(2) Use the options on the OPTIONS card as shown in table 9.

(3) If all else fails, modify the models file. Perhaps a different set of parameters is needed or the values for the existing parameters are unacceptable. For comparison, refer to the models file included in table 9.

(4) For additional help, consult reference [9].

\subsection{STAT2}

STAT2 is used to determine correlation coefficients $[3,10,11]$ and to generate wafer maps for KEYS. The sample correlation coefficient, $r$, is a measure of the similarity of the spatial variation of two sets of data. Consider the paired observations $\left(x_{1}, y_{1}\right),\left(x_{2}, y_{2}\right)$, $\ldots,\left(x_{n}, y_{n}\right)$ on two quantities. If a large value of $x_{i}$ implies a large value of $y_{i}$, then the quantities are said to be positively correlated, and $r$ is positive. If a large value of $x_{i}$ implies a small value of $y_{i}$, then the quantities are said to be negatively correlated, and $r$ is negative. If a large value of $x_{i}$ implies nothing about $y_{i}$, then $x$ and $y$ are said to be 
uncorrelated, and $r$ is near zero. The statistic $r$ is given as follows:

$$
r=\frac{\sum_{i=1}^{n}\left(x_{i}-\bar{x}\right)\left(y_{i}-\bar{y}\right)}{\sqrt{\left[\sum_{i=1}^{n}\left(x_{i}-\bar{x}\right)^{2}\right]\left[\sum_{i=1}^{n}\left(y_{i}-\bar{y}\right)^{2}\right]}}
$$

where $\bar{x}$ and $\bar{y}$ are the arithmetic means of the $x_{i}$ and $y_{i}$ values of the sample summed over the $n$ points. Note that $r$ must take on values in the range $[-1,1]$. Stronger positive or negative correlation is indicated by $r$ approaching +1 or -1 , respectively.

The output of SUXES produces a set of files readable by STAT2. There is one file for each parameter of interest. An example of such a STAT2-readable file is shown in table 10. Site location information can be understood with the help of figure 3 . The first 16 records of the file contain the location information for the data values which follow. The first of the 16 records gives the location of the first sites in rows 1 through 4. The second record gives the location of the last sites in rows 1 through 4 . In the example of table 10, the first row of the wafer has test sites in columns 4 through 6 , the second row of the wafer has test sites in columns 2 through 8 , etc. The third and fourth records give the location of the first and last site in row 5 . When all rows have been characterized, remaining positions within the first 16 records of the file contain zeroes. The data values are given in records 17 through the end of the file. They are ordered by rows, such that values from the first row of sites are first, followed by values from the second row of sites, and so on for as many rows of sites as are present.

In order for STAT2 to calculate correlation coefficients, it is necessary to create a database and enter the processed output of SUXES into the database. The database is created by the CRDB program (a program which is adjunct to and supplied with STAT2) [3] such that data from all test sites are included in the database. A sequence of commands such as that shown in table 11 directs STAT2 to enter the processed output of SUXES into the database. Each of the STAT2-readable files is assigned and read (STAT2 commands ASG and REA), values less than $10^{-12}$ are excluded (XLT), statistics are calculated for the remaining sites (PRS), replacement values are calculated for the excluded sites (AXP), and the data set is written to the database (WDB). A total of 43 parameters are thus processed. Because the first record in the database serves as a directory, writing of actual data begins at record 2 or entry number 2 and ends at entry number 44.

Having constructed the database, correlation coefficients are calculated using the STAT2 GET and SDB commands. As a default, the measured frequency, simulated frequency, and the percent difference are used as reference data sets (database entries 4, 5, and 6) with which all other data sets are correlated. The resulting correlation coefficients are sorted in KEYS by magnitude. Table 12 contains an example of the output. This table shows at a glance the parameters which are most influential in determining the measured frequency, simulated frequency, and percent difference.

It is often useful to see the spatial variation of one or more parameters over the wafer. Using a sequence of commands such as that shown in table 13, STAT2 can produce gray-tone maps similar to the one shown in figure 4. 
Table 10. Example of a File Suitable for Input to STAT2*

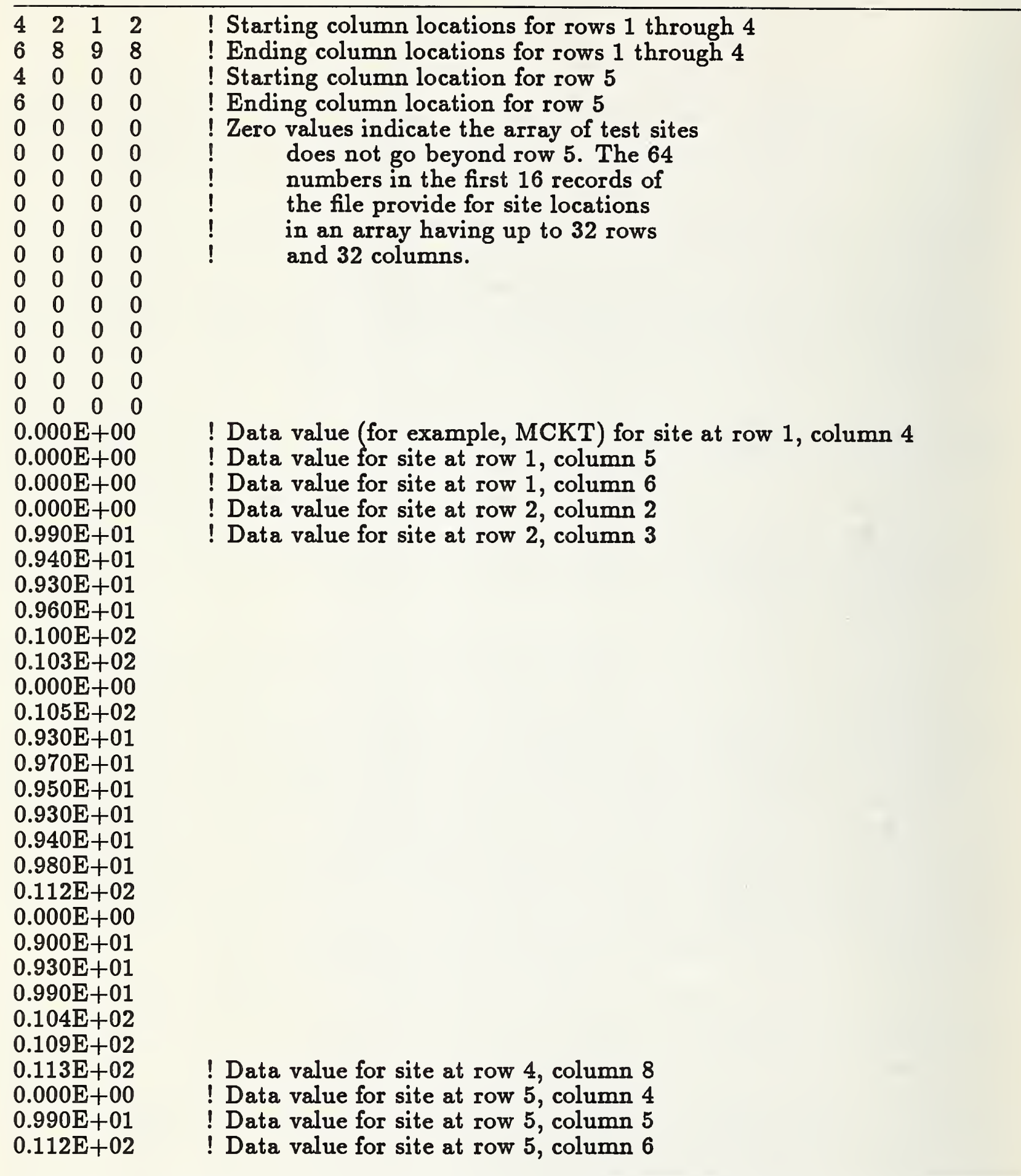

*Text beginning with an exclamation point is explanatory and not part of the actual file. The original file had nine rows of sites. In the interest of brevity the table shows an example having five rows and nine columns as shown in figure 3. 


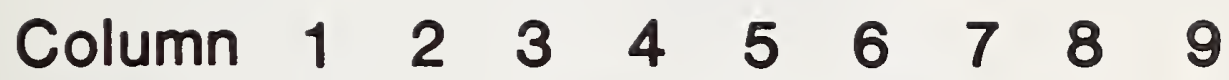

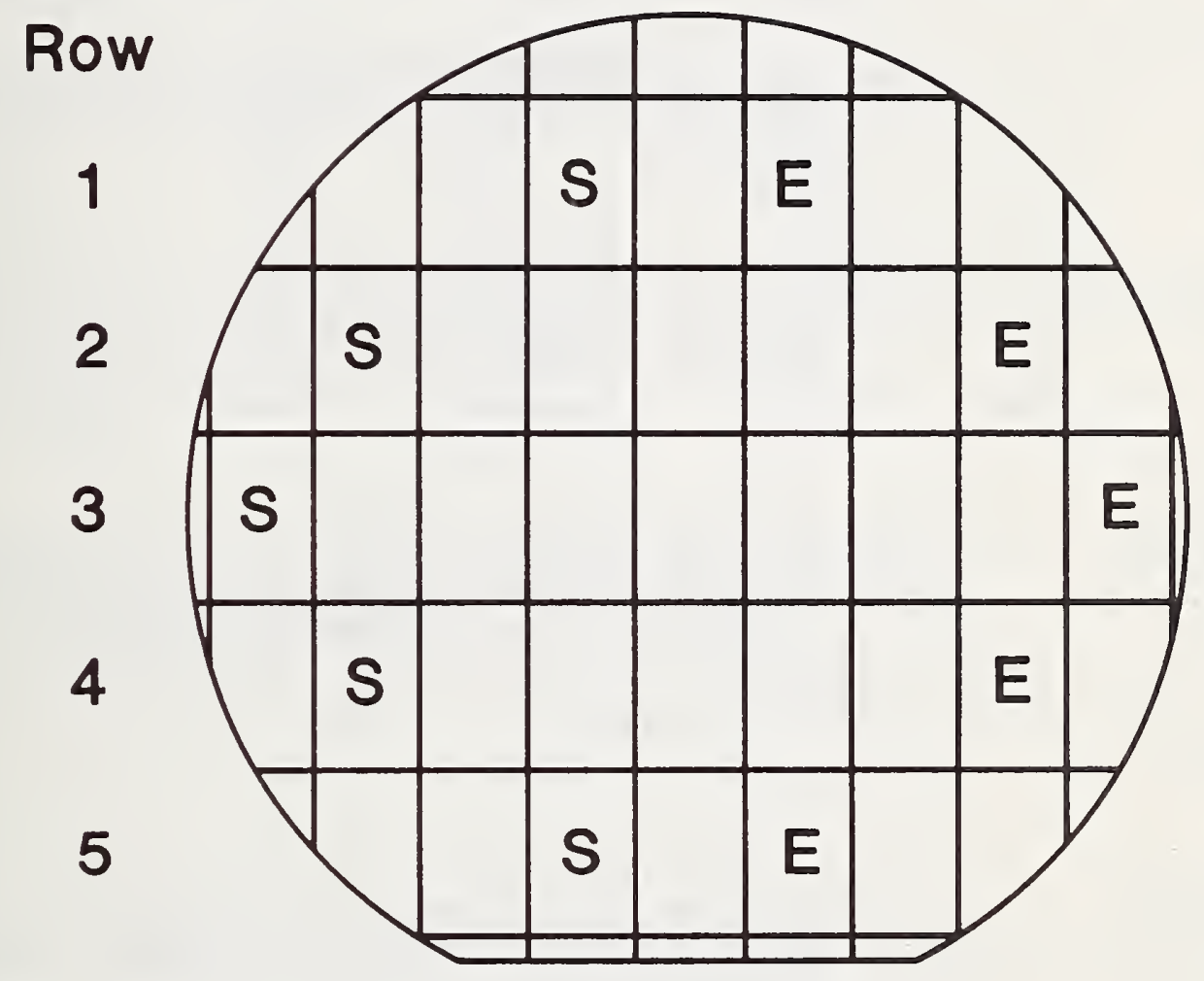

Figure 3. Schematic grid for STAT2 nomenclature regarding site locations. 
Table 11. Example of a STAT2 Command Sequence Used to Build a Database and Calculate Correlation Coefficients*

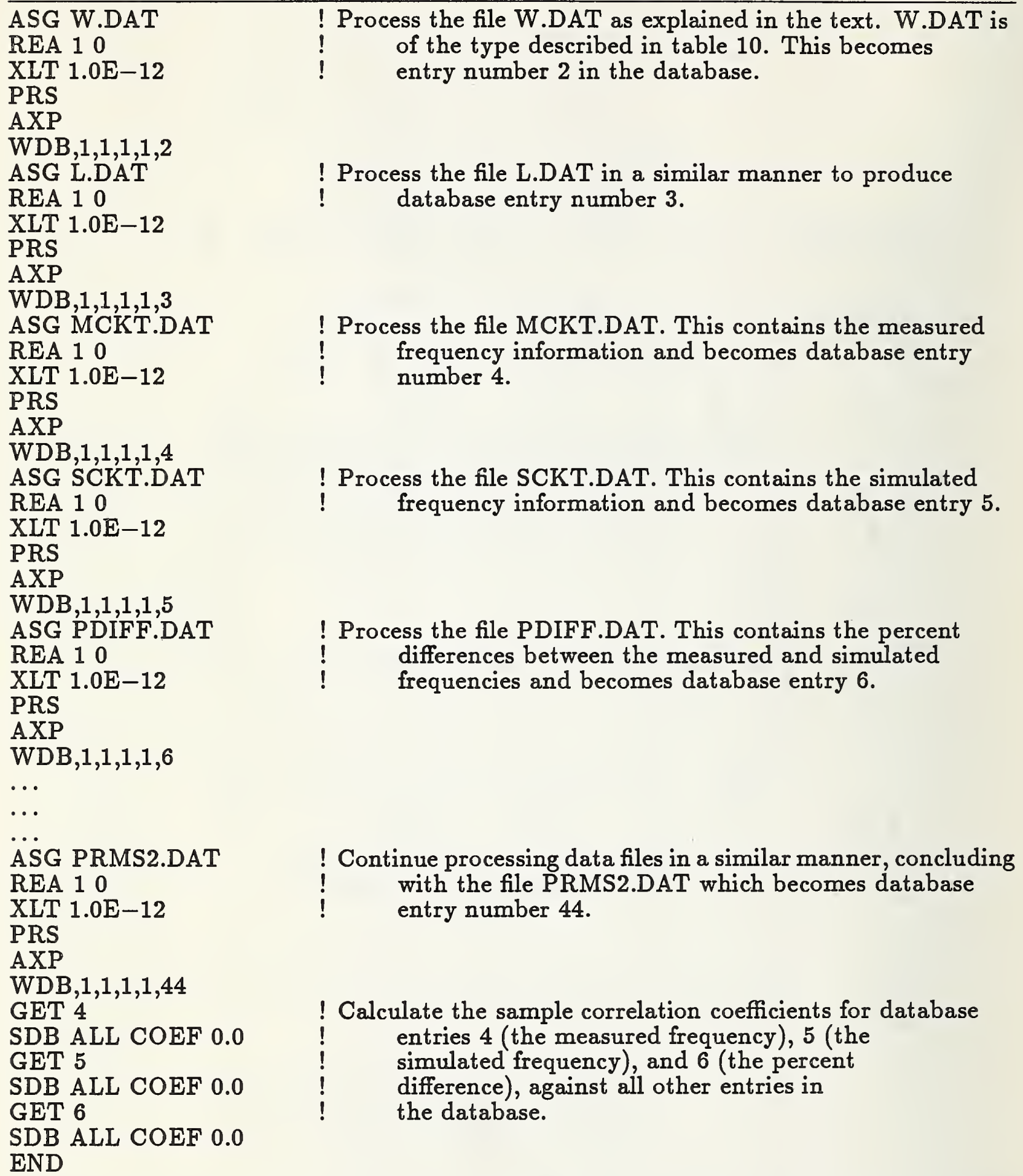

! Process the file L.DAT in a similar manner to produce ! database entry number 3.

! Process the file MCKT.DAT. This contains the measured ! frequency information and becomes database entry

! Process the file PDIFF.DAT. This contains the percent ! differences between the measured and simulated ! frequencies and becomes database entry 6.

! Continue processing data files in a similar manner, concluding ! with the file PRMS2.DAT which becomes database ! entry number 44 .

! Calculate the sample correlation coefficients for database ! entries 4 (the measured frequency), 5 (the ! simulated frequency), and 6 (the percent ! difference), against all other entries in ! the database.

*Text beginning with an exclamation point is explanatory and not part of the actual file. 
Table 12. A Rank-Ordering of the Magnitudes of the Ten Highest Correlated Parameters with Respect to (a) the Percent Difference Between the Measured and Simulated Ring Oscillator Frequencies, (b) the Measured Frequency, and (c) the Simulated Frequency. An $n$ - or $p$-channel device designation appears before most of the parameters to distinguish between the device types.

\begin{tabular}{|c|c|c|c|c|c|c|}
\hline & $\begin{array}{l}\text { (a) T1 } \\
\text { Perce } \\
\text { Differ }\end{array}$ & & $\begin{array}{l}\text { (b) Th } \\
\text { Measu } \\
\text { Freque }\end{array}$ & & $\begin{array}{l}\text { (c) The } \\
\text { Simulat } \\
\text { Frequen }\end{array}$ & \\
\hline & Parameter* & $\begin{array}{l}\text { Corr. } \\
\text { Coef. }\end{array}$ & Parameter** & $\begin{array}{l}\text { Corr. } \\
\text { Coef. }\end{array}$ & Parameter & $\begin{array}{l}\text { Corr. } \\
\text { Coef. }\end{array}$ \\
\hline $\begin{array}{l}1 \\
2 \\
3 \\
4 \\
5 \\
6 \\
7 \\
8 \\
9 \\
10\end{array}$ & $\begin{array}{l}\text { PDIFF } \\
\text { NKP } \\
\text { NUO } \\
\text { SCKT } \\
\text { PKP } \\
\text { NLD } \\
\text { NCGBO } \\
\text { NSUB } \\
\text { NCGDO } \\
\text { NGAMMA }\end{array}$ & $\begin{array}{l}1.000 \\
0.915 \\
0.909 \\
0.886 \\
0.809 \\
0.730 \\
0.706 \\
0.701 \\
0.698 \\
0.672\end{array}$ & $\begin{array}{l}\text { MCKT } \\
\text { SCKT } \\
\text { NKP } \\
\text { NUO } \\
\text { NKAPPA } \\
\text { PKP } \\
\text { PTOX } \\
\text { NSUB } \\
\text { NGAMMA } \\
\text { NLD }\end{array}$ & $\begin{array}{l}1.000 \\
0.745 \\
0.660 \\
0.659 \\
0.618 \\
0.616 \\
0.511 \\
0.451 \\
0.404 \\
0.385\end{array}$ & $\begin{array}{l}\text { SCKT } \\
\text { NKP } \\
\text { NUO } \\
\text { PDIFF } \\
\text { PKP } \\
\text { MCKT } \\
\text { NSUB } \\
\text { NLD } \\
\text { NCGBO } \\
\text { NCGDO }\end{array}$ & $\begin{array}{l}1.000 \\
0.979 \\
0.972 \\
0.886 \\
0.877 \\
0.745 \\
0.713 \\
0.698 \\
0.678 \\
0.670\end{array}$ \\
\hline
\end{tabular}

*Remodeling these parameters can change the percent difference between the measured and simulated ring oscillator frequencies.

**Wafer maps of these parameters may suggest strategies to improve process control.

Table 13. Example of a STAT2 Command Sequence Used to Make a Gray-Tone Map

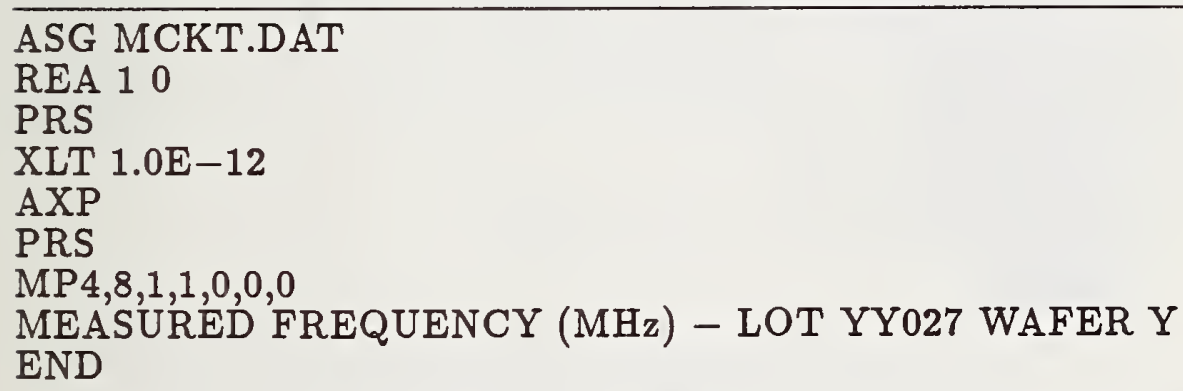




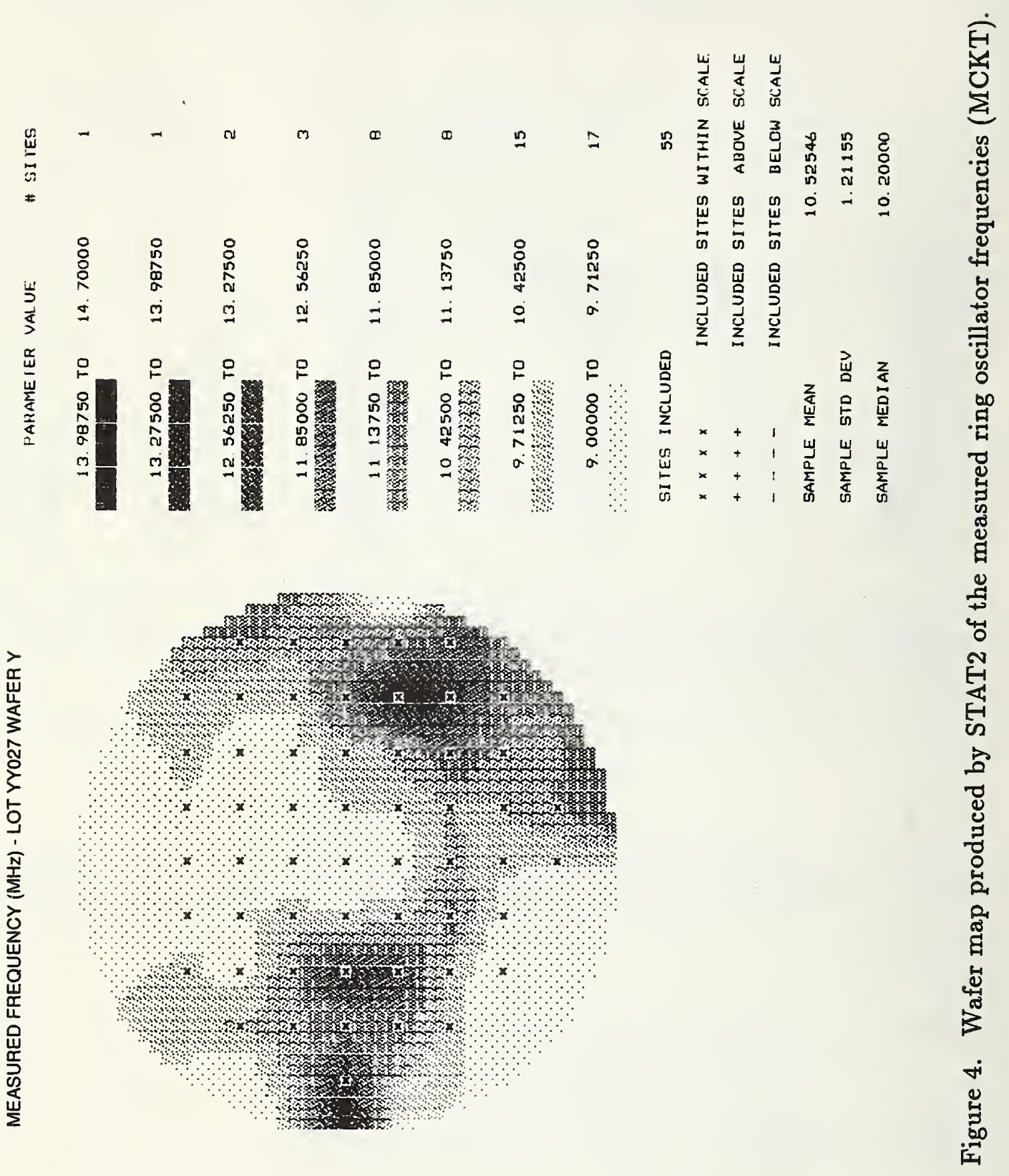




\section{AN EXAMPLE USING CMOS TECHNOLOGY}

In this part of this report, an example of the application of KEYS is presented. In particular, a twin-tub, n-substrate, CMOS bulk process using four-micrometer design rules was used in this example to manufacture 65 test chips on a 4 -in. $(10.16-\mathrm{cm})$ wafer. A 19-stage ring oscillator was selected as the demonstration circuit. MOSFETs, both $n$ and p-channel, with the same dimensions as the ring oscillator MOSFETs, were used to measure the I-V data points for SUXES.

This part is divided into two sections. Section 3.1 describes the test structure measurement methods and section 3.2 discusses the output which consists of plots, tabular listings, and wafer maps.

\subsection{Test Structure Measurement Methods}

Three types of test structures were probed on each test chip: a ring oscillator, cross-bridge resistors $[12,13]$, and $n$ - and p-channel MOSFETs with the same dimensions as the ring oscillator MOSFETs. A manual probe station and oscilloscope were used to probe the ring oscillators, and the measurements for the other test structures were taken with an Accutest Model 3000 parametric test system.

The ring oscillator was selected as the demonstration circuit. It is a simple circuit and can be used to compare frequencies among chips. Since the frequency is sensitive to the model parameters, it is also a measure of how "reasonable" the critical parameters are to the user. Critical parameters are defined here to be those parameters which have the greatest effect on the ring oscillator frequency [8]. If the user is not satisfied with the percent difference between the simulated and measured frequencies, then the value of a critical parameter is considered "unreasonable" to the user.

The 19-stage ring oscillator is shown in figure 5. The designed channel lengths of the $n$ - and p-channel MOSFETs and the designed channel widths of the $n$-channel MOSFETs are 4 $\mu \mathrm{m}$. The designed channel widths of the $p$-channel MOSFETs are $8 \mu \mathrm{m}$. A cross-sectional view of an inverter is shown in figure 6 . The $n$-wells are not contacted on the top side of the wafer, creating a small voltage drop (approximately $0.1 \mathrm{~V}$ ). This is due to the doping differences between the substrate, set at $5.0 \mathrm{~V}$, and the $n$-well. A crude estimate of this voltage drop assumes an abrupt change in the doping concentrations between the $n$-type well and $n$-type substrate. This estimate is:

$$
\text { voltage drop }=\left(\frac{\mathrm{kT}}{\mathrm{q}}\right)\left[\ln \left(\frac{\mathrm{NWELL}}{\mathrm{SUB}}\right)\right] \text {, }
$$

where NWELL is the $n$-well doping concentration, and SUB is the $n$-substrate doping concentration. This estimate for the voltage drop decreases the simulated ring oscillator frequency by $0.3 \%$. To minimize interference with the actual signal, low capacitance probes $(0.01 \mathrm{pF})$ are used to probe the output of the ring oscillator. Care was taken to minimize effects that could alter the measured results. A $5-\mathrm{V}$ voltage swing was obtained. Start-up circuitry is not incorporated into the design of this ring oscillator; however, a comparison 


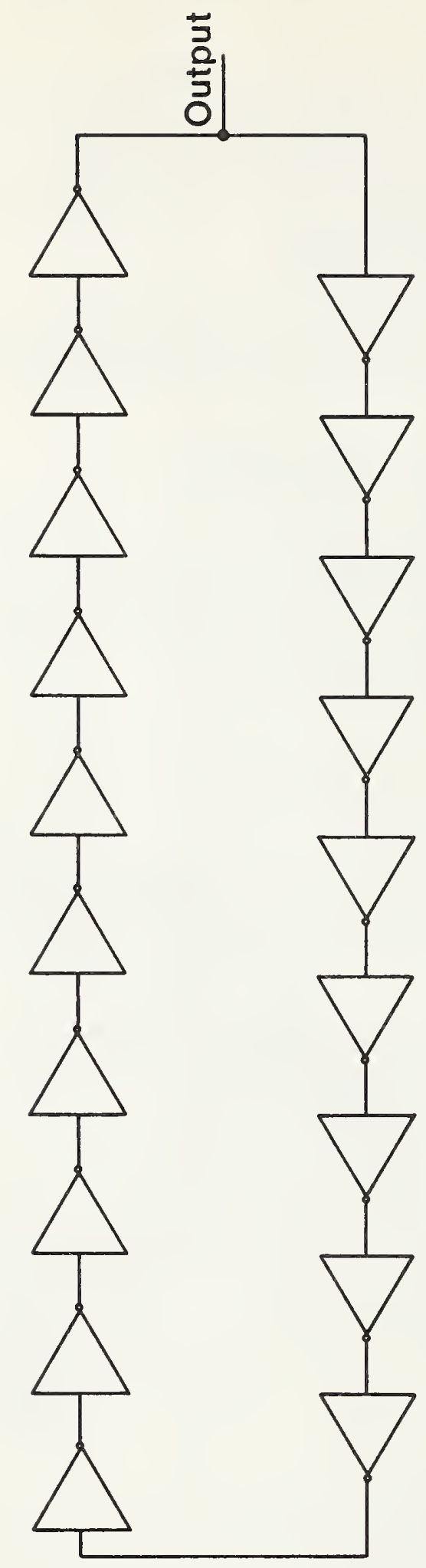

Figure 5. Block diagram of the 19-stage CMOS ring oscillator. 


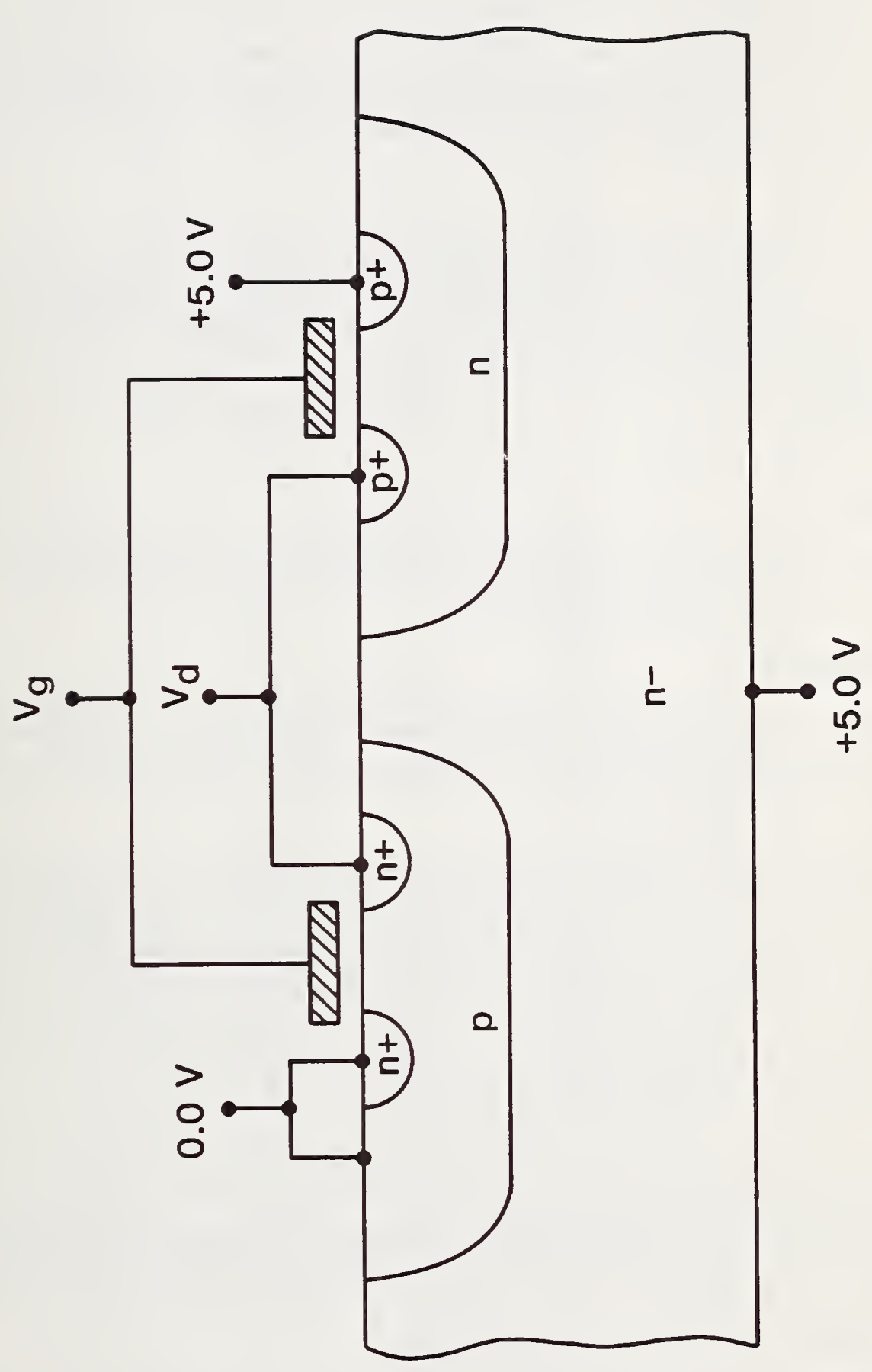

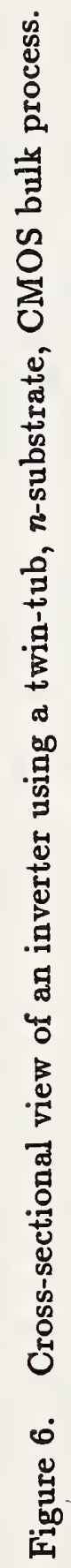


of the measured waveforms with the simulated waveforms reveals that the fundamental oscillation frequency is obtained.

Four-micrometer cross-bridge resistors were probed automatically to determine sheet resistance and linewidth values for $n^{+}$diffusion, poly, and metal. A cross-bridge resistor is shown in figure 7. For details on how to obtain the sheet resistance and linewidth values from these test structures, see references $[12,13]$. The polysilicon linewidth values obtained from this measurement are representative of the magnitude and changes in the MOSFET channel-lengths used in SPICE.

The third group of test structures are the $n$ - and $p$-channel MOSFETs with the same dimensions as the ring oscillator MOSFETs. These devices, of which the $p$-channel MOSFET is shown in figure 8, were used to measure the I-V data points for SUXES and to obtain the initial estimates for the parameters NSUB, VTO, KP, GAMMA, PHI, and UO. These parameters are defined in table 2. The voltage values used to obtain these measurements are given in table 14.

\subsection{The Output}

KEYS can produce output in the form of plots, tabular listings, and wafer maps. Each form is discussed in the subsections that follow.

\subsubsection{The Output Plots}

For each chip, plots are made for the $n$ - and $p$-channel MOSFET I-V characteristics and a simulated ring oscillator node potential as a function of time. These plots verify that the parameter values are satisfactory.

The I-V characteristics include three separate sets of curves: the measured I-V data, SUXES's predicted I-V data, and SPICE's simulated I-V data. Any deviations will be visually apparent to the circuit designer. A plot for a $p$-channel MOSFET is shown in figure 9. The open circles refer to the measured I-V data points. SUXES predicts the I-V data points using its optimized parameters in SPICE, and the symbol for these data is a filled circle. Finally, a solid line refers to the simulated I-V data points obtained from SPICE using the models file discussed in section 2.2.

For the whole wafer, good agreement is seen among the three curves for the p-channel MOSFETs. This is also the case for the $n$-channel MOSFETs. For the wafer, the average percent deviation, for all the acceptable chips, between the measured I-V data points and SUXES's predicted I-V data points for the p-channel MOSFETs is $3.2 \%$. This value is $3.4 \%$ for the $n$-channel MOSFETs. An acceptable chip is one whose optimized SUXES values are not pegged at the lower or upper bounds.

In KEYS, SPICE is also used to simulate the response of an optional dynamic circuit. The measured waveforms are compared with the SPICE simulated waveforms. Figure 10 displays a simulated ring oscillator waveform. The frequency and period are also recorded on these plots. The ring oscillator is oscillating in its fundamental mode [14] and the node 


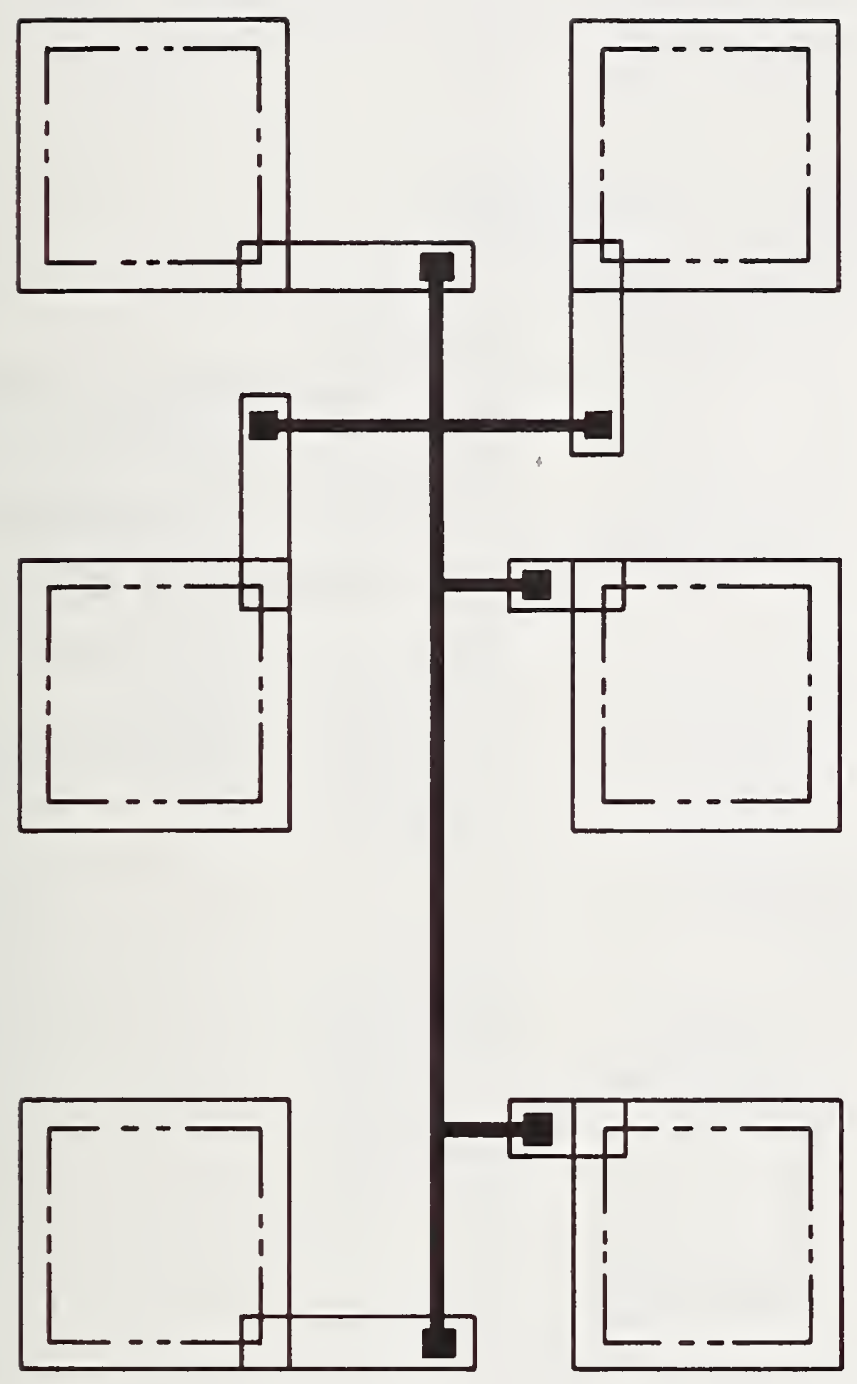

Polysilicon

- Contact

- Metal

Figure 7. Four- $\mu \mathrm{m}$ cross-bridge resistor for determining the polysilicon linewidth. 

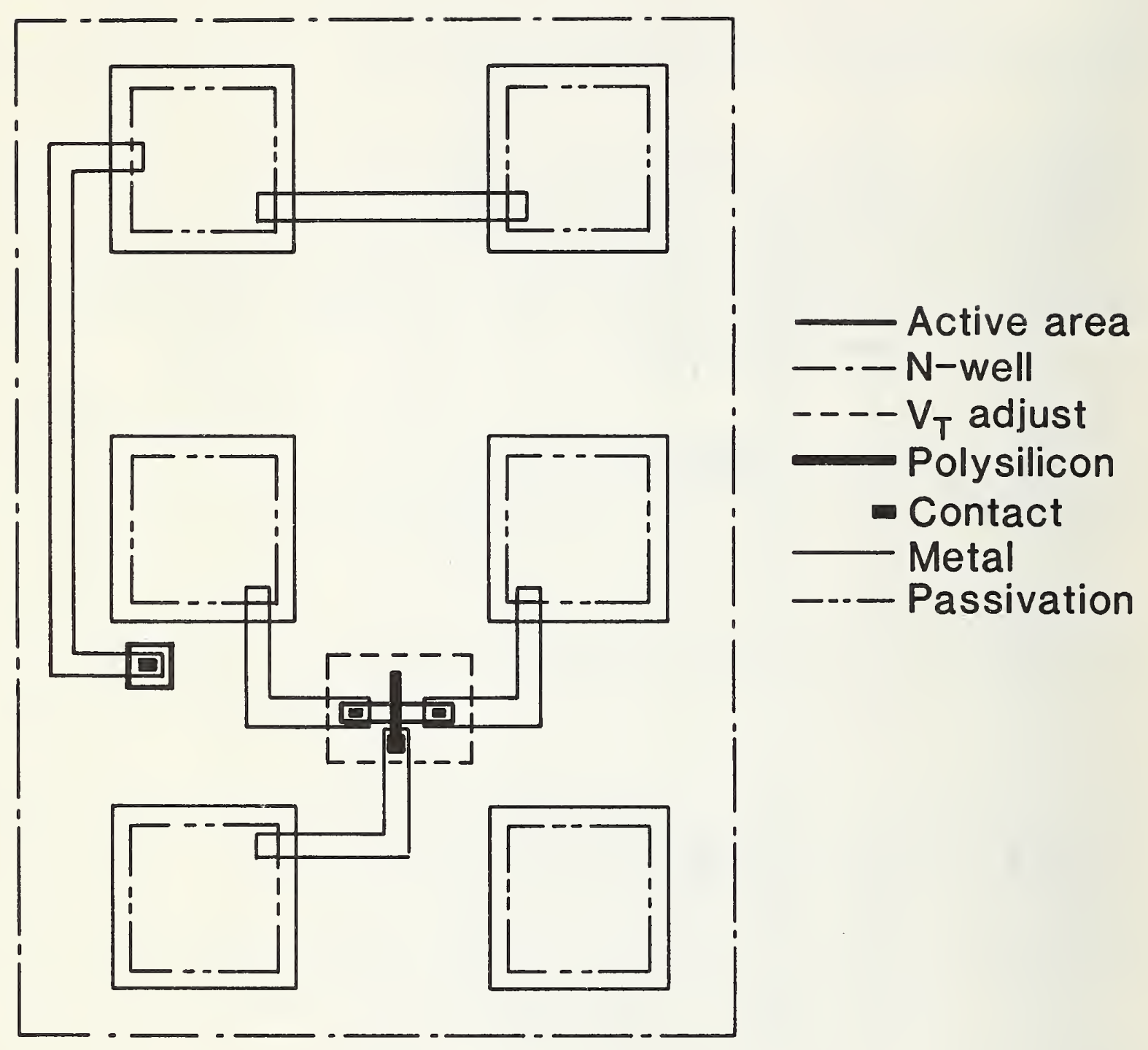

Figure 8. P-channel MOSFET of the same dimensions as the $p$-channel ring oscillator MOSFETs. 
Table 14. The Voltage Values Used to Measure the $N$ - and $P$-Channel MOSFETS

I-V Data Point

Measurements
Measurements

Needed for the

Initial Estimates

\begin{tabular}{|c|c|c|c|c|}
\hline & n-channel & p-channel & $n$-channel & $p$-channel \\
\hline $\begin{array}{l}\text { Device } \\
\text { dimensions }\end{array}$ & $\begin{array}{c}\mathrm{L}_{\mathrm{n}}=4 \mu \mathrm{m} \\
\mathrm{W}_{\mathrm{n}}=4 \mu \mathrm{m}\end{array}$ & $\begin{array}{c}\mathrm{L}_{\mathrm{p}}=4 \mu \mathrm{m} \\
\mathrm{W}_{\mathrm{P}}=8 \mu \mathrm{m}\end{array}$ & $\begin{array}{c}\mathrm{L}_{\mathrm{n}}=4 \mu \mathrm{m} \\
\mathrm{W}_{\mathrm{n}}=4 \mu \mathrm{m}\end{array}$ & $\begin{array}{c}\mathrm{L}_{\mathrm{p}}=4 \mu \mathrm{m} \\
\mathrm{W}_{\mathrm{p}}=8 \mu \mathrm{m}\end{array}$ \\
\hline$V_{b s}$ & $0.0 \mathrm{~V}$ & $0.0 \mathrm{~V}$ & $0,-3,-6,-9 \mathrm{~V}$ & $0,3,6,9 \mathrm{~V}$ \\
\hline$V_{g s}$ & $\begin{array}{c}0.1,0.2,0.3 \\
0.5,1,1.5 \\
2,2.5,3, \\
3.5,4,4.5 \\
\text { and } 5 \mathrm{~V}\end{array}$ & $\begin{array}{c}-0.1,-0.2 \\
-0.3,-0.5 \\
-1,-1.5 \\
-2,-2.5 \\
-3,-3.5 \\
-4,-4.5 \\
\text { and }-5 \mathrm{~V}\end{array}$ & $\begin{array}{c}\text { not } \\
\text { applicable }\end{array}$ & $\begin{array}{c}\text { not } \\
\text { applicable }\end{array}$ \\
\hline $\mathrm{V}_{\mathrm{ds}}$ & $\begin{array}{c}0.1,0.2,0.3 \\
0.5,1,1.5 \\
2,2.5,3 \\
3.5,4,4.5 \\
\text { and } 5 \mathrm{~V}\end{array}$ & $\begin{array}{c}-0.1,-0.2 \\
-0.3,-0.5 \\
-1,-1.5 \\
-2,-2.5 \\
-3,-3.5 \\
-4,-4.5 \\
\text { and }-5 \mathrm{~V}\end{array}$ & $0.2 \mathrm{~V}$ & $-0.2 \mathrm{~V}$ \\
\hline $\mathrm{V}_{\text {chuck }}$ & $0.0 \mathrm{~V}$ & $0.0 \mathrm{~V}$ & $0.0 \mathrm{~V}$ & $V_{b s}$ \\
\hline
\end{tabular}




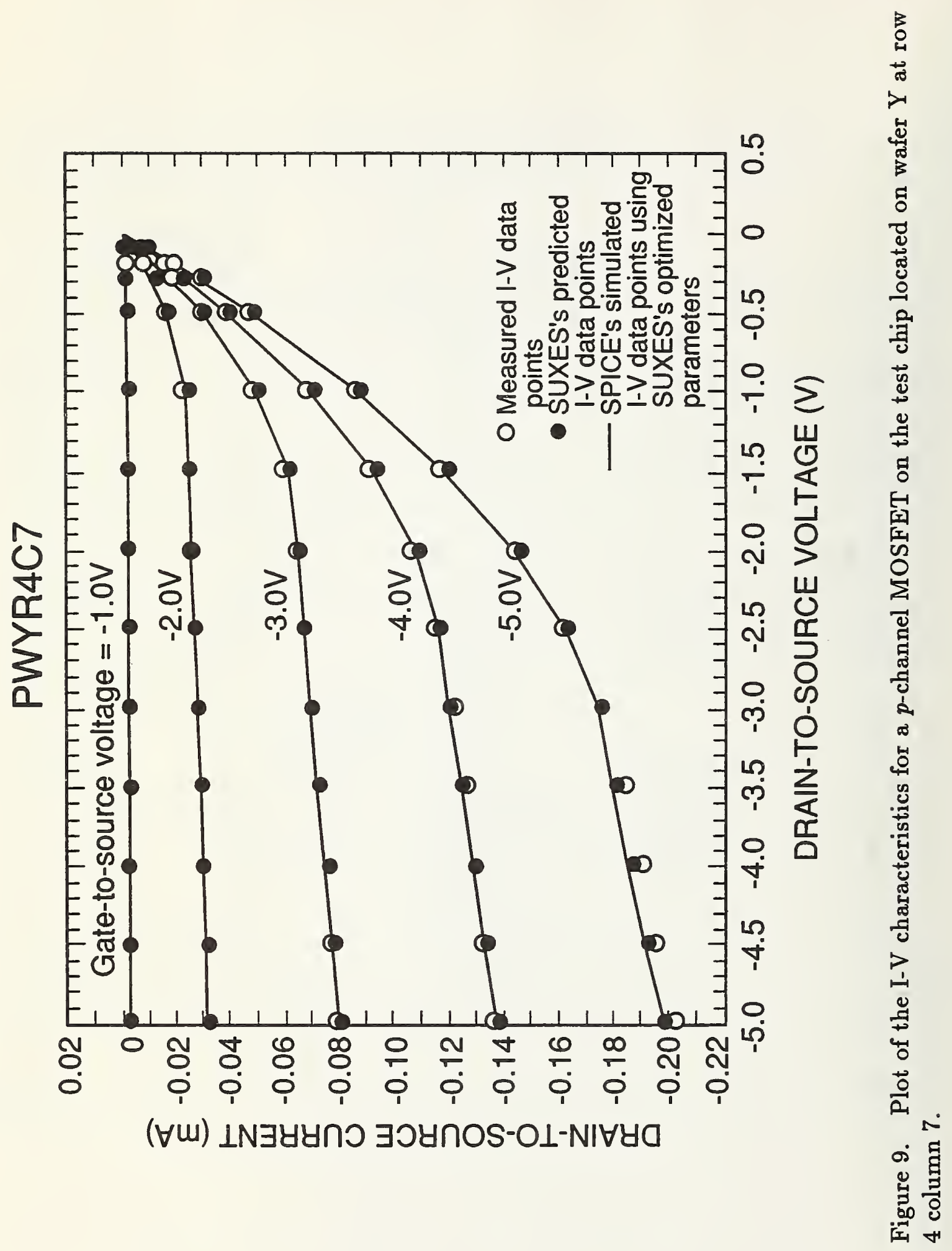




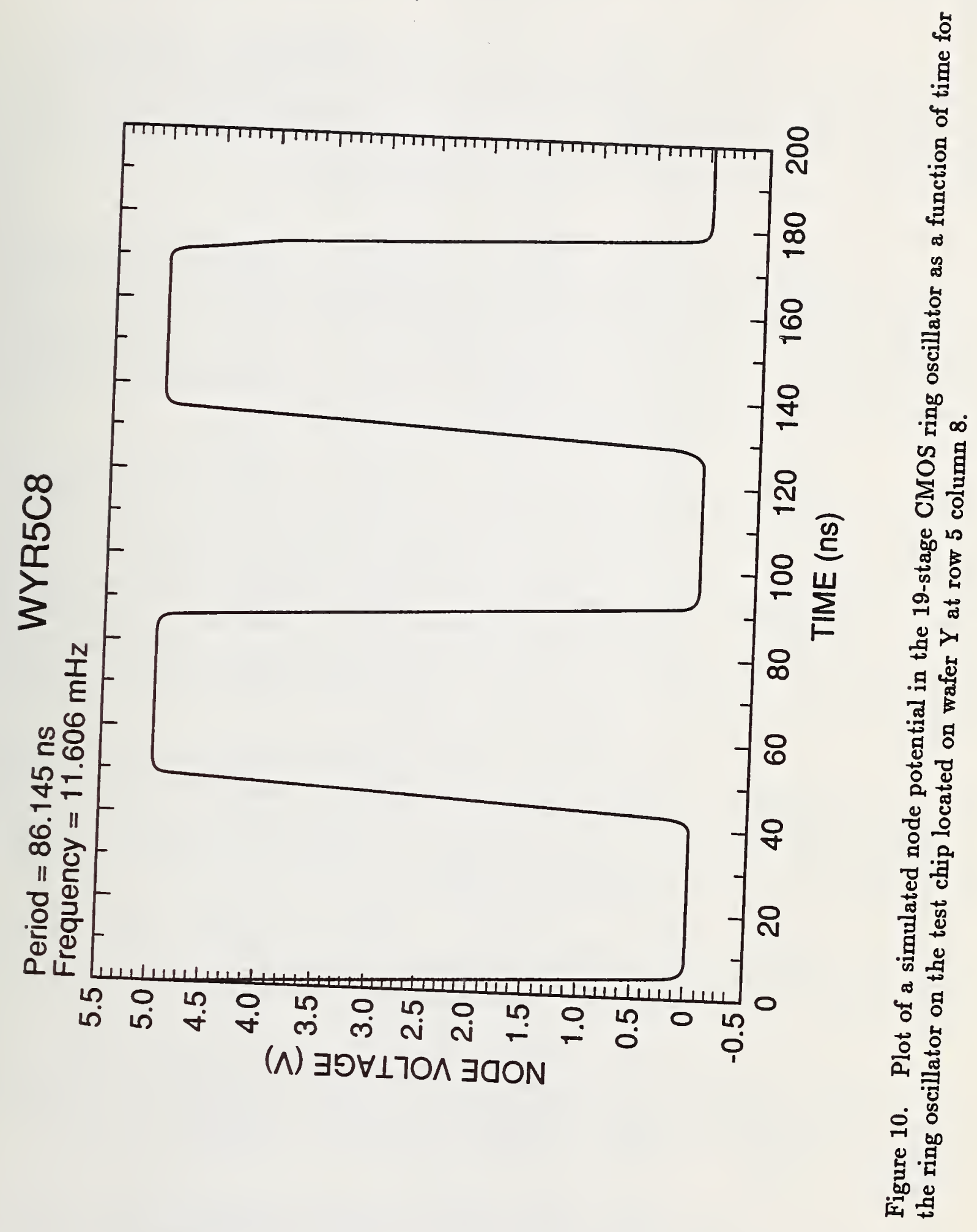


voltages undergo a $5-\mathrm{V}$ voltage swing. For the wafer, the average percent difference, for all the acceptable chips, between the simulated and measured ring oscillator frequencies was $8.6 \%$.

The titles for figures 9 and 10 indicate the wafer and the chip location on the wafer. For the I-V characteristics, an $n$ - or $p$-channel device designation is included. For example, PWYR4C7 is a plot (fig. 9) of the I-V characteristics for a $p$-channel MOSFET on the chip located at row 4 column 7 on wafer Y, and WYR5C8 is a plot (fig. 10) of a ring oscillator node potential on the chip located at row 5 column 8 on wafer $Y$.

\subsubsection{The Tabular Listings}

KEYS generates two types of tabular listings: a comprehensive summary and rank-orderings of the correlation coefficients. The comprehensive summary is shown in table 15 . This table provides the measurement results, the optimized values of SUXES's parameters, the simulation results, the calculated parameters for each chip, the wafer averages, and the lot averages when appropriate. The comprehensive summary in combination with the plots verifies that the I-V data, SUXES, and SPICE are working properly.

Preceding the results from each chip, a "+" or "-_" sign appears. A chip's results are either accepted or rejected. If the values for the $n$ - and $p$-channel MOSFETs lie between specified lower and upper bounds in SUXES's initial estimates file, it is considered an "acceptable" chip as defined by the user. Preceding the results of an "acceptable" chip, a "+" sign appears. If a parameter's value is pegged at one of these boundaries, then preceeding the results for that chip, a "-" sign appears. Assuming the I-V data look reasonable to the user, a rejected chip can become an accepted chip by modifying the strategy file. This is described in Appendix B. Chips with unreasonable linewidth and frequency measurements as determined by the user are removed from the database.

The wafer averages follow the results from all the chips. The number of acceptable chips out of the total number of chips is recorded. The format of the wafer averages is similar to the format for a single chip. The values are the averages of the corresponding values from the acceptable chips. Throughout this table, the $n$-channel MOSFET information is listed before the $p$-channel MOSFET information.

The lot averages, when appropriate, follow the results from the last wafer. The total number of wafers is recorded. The format for the lot averages is similar to that of the wafer averages. The values are the corresponding average values from the wafer averages.

For brevity, table 15 provides the results from five chips as opposed to all the chips. Hence, in the complete listing, the average values would correspond to the wafer averages. For the entire wafer, the average percent difference between the simulated and measured ring oscillator frequencies was $8.6 \%$. Different strategy files can change this average percent difference.

The second type of tabular listing is a rank-ordering of the spatial correlation coefficients with respect to chosen parameters. The rank-orderings for this example are given in 
Table 15. A Tabular Summary of Each Chip's Results Similar to the Wafer Summary. For brevity, this listing was made for five chips. The $n$-channel MOSFET information is listed before the $p$-channel MOSFET information.

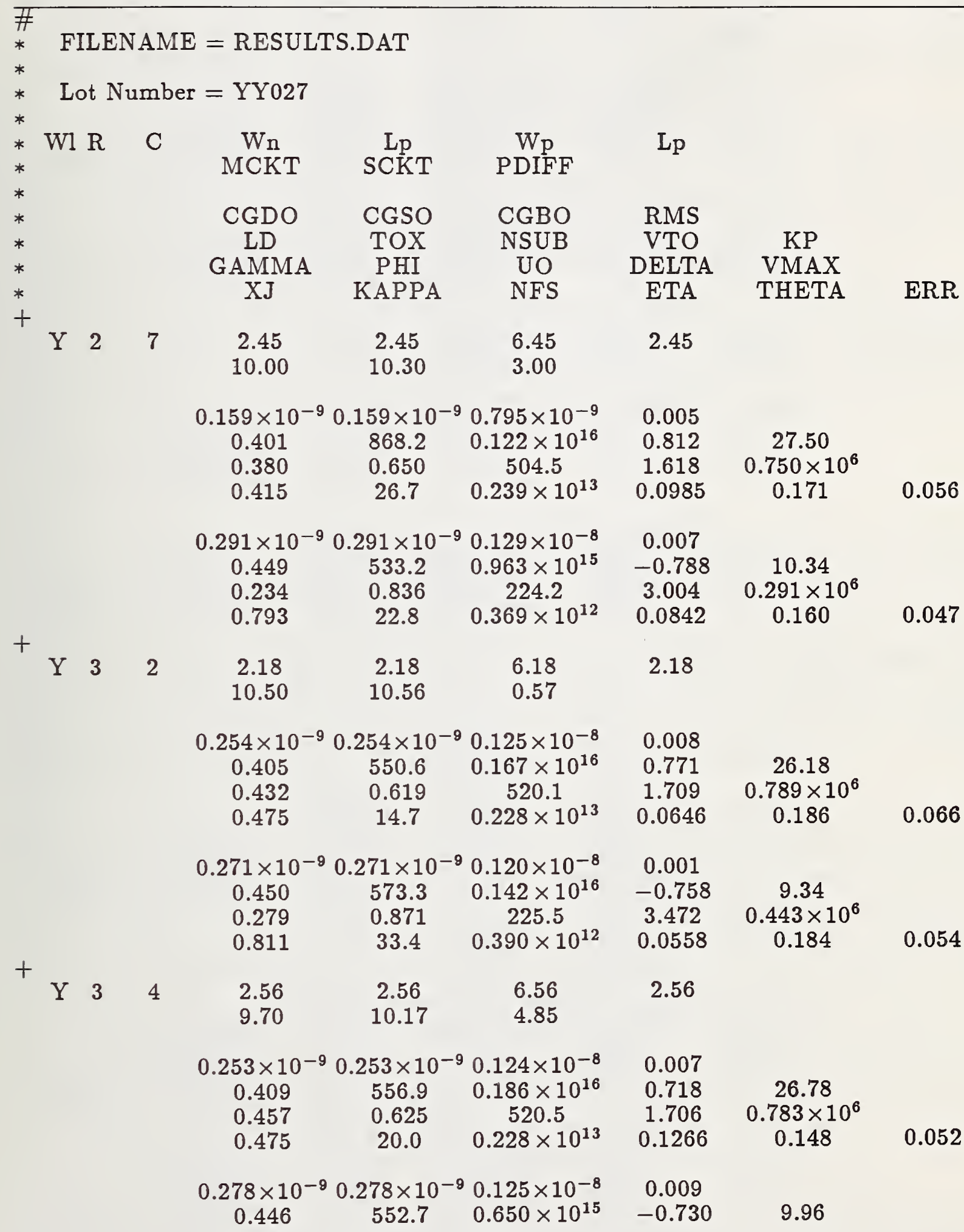




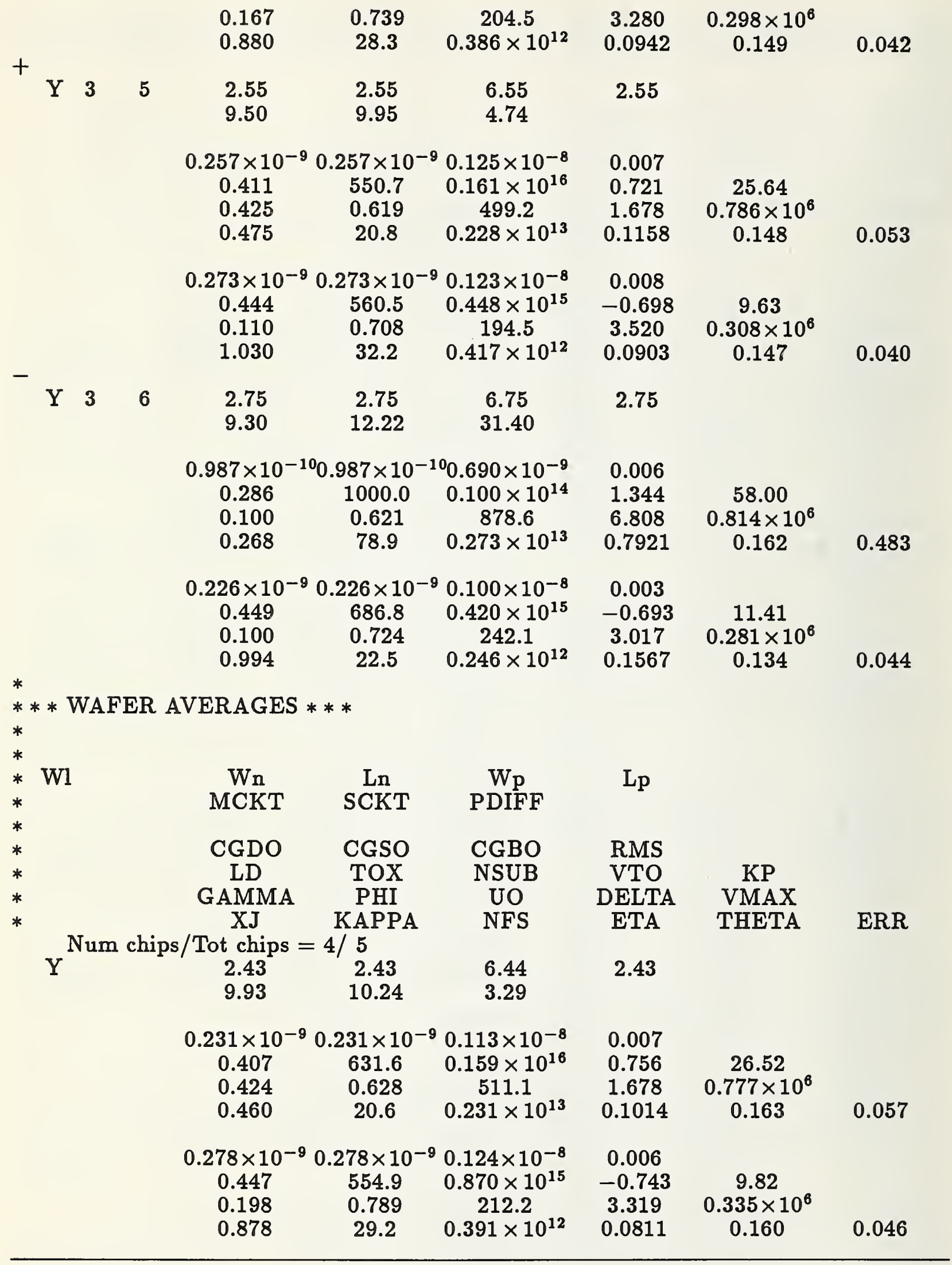


table 12. These listings are with respect to the following three chosen parameters: the percent difference between the measured and simulated ring oscillator frequencies (PDIFF), the measured ring oscillator frequency (MCKT), and the simulated ring oscillator frequency (SCKT). In these tables, an $n$ - or $p$-channel device designation appears before most of the parameters to distinguish between the device types. The exceptions are the parameters which do not require a designation. They are: PDIFF, MCKT, and SCKT.

The parameters at the top of the rank-ordering in table 12(a), if reoptimized, can change PDIFF. To reoptimize the parameters, the strategy file may be altered according to the procedure given in Appendix B. Remodeling these same parameters can also change PDIFF. This could have the effect of decreasing the rms deviation between the measured and predicted I-V data points, and it could increase the number of acceptable chips.

Table 12(b) lists the correlation coefficient of each parameter with respect to MCKT. Given a minimum number of acceptable chips (as predetermined by the user), a wafer map of MCKT and wafer maps of the highly correlated parameters with respect to MCKT may suggest strategies to improve process control. An example of a wafer map is given in figure 4 for MCKT. This is the third form of output.

\subsubsection{The Wafer Maps}

Wafer maps may be generated from the results of the correlation coefficient rank-orderings. If a minimum number of acceptable chips (as predetermined by the user) are obtained, the wafer maps are generated by STAT2. In this example, wafer maps are made of the following parameters: (1) MCKT; (2) SCKT; (3) PDIFF; (4) the two highest correlated parameters with respect to PDIFF; excluding the maps already generated; and (5) the two highest correlated parameters with respect to $\mathrm{MCKT}$, excluding the maps already generated. An example of a wafer map is given in figure 4 for the parameter MCKT. This map along with wafer maps of the highly correlated parameters with respect to MCKT may suggest strategies to improve process control.

The user determines the minimum number of acceptable chips. If this value is too low, meaningless wafer maps can be generated. Altering the strategy file (using the procedure in Appendix B) for the parameters at the top of table 12(a) may increase the number of acceptable chips. Other techniques may be just as viable. Meaningful wafer maps are an invaluable tool in evaluating and improving the process. Distinctive patterns may exist which may be caused by limitations in the processing equipment or the manner in which such equipment is used to handle wafers. For example, the doping is at a minimum at the center of the wafer when it is placed in the middle of a fully loaded, slotted quartz diffusion boat. In this case, the flow of gas is maintained parallel to the face of the wafer. When this flow is not parallel to the face of the wafer, other patterns may result. One example is two doping minima [15].

\section{CONCLUSIONS}

This report describes a computer procedure called KEYS that can be used to characterize 
a chip, wafer, or lot. KEYS links SUXES, SPICE, and STAT2 according to the flowchart in figure 2. The software used in KEYS is given in table 1.

The benefits of this procedure are as follows: (1) the technique of running SUXES and SPICE becomes standardized; (2) a considerable amount of time is saved; (3) manual intervention and error are reduced; (4) the procedure can be modified for different dynamic circuits; (5) the positional information and the comprehensive output are valuable to process engineers, modelers, and designers; and (6) modifying KEYS for updated versions of SUXES, SPICE, and STAT2 is relatively easy as long as the authors of these programs do not modify their input-output formats.

An example using CMOS technology is also given. Some of the limitations of this procedure for the example are as follows: (1) the program aborts if the I-V data are not characteristic of a typical MOSFET; (2) the boundary values in SUXES are arbitrary; (3) the user determines the minimum number of acceptable chips, and meaningless wafer maps are an indication this value is too low; (4) considerable effort is required to modify the strategy file, according to the procedure given in Appendix $B$, to increase the number of acceptable chips; and (5) the user helps to determine, via the strategy file, how closely the simulated results match the measured results.

The output of this software addresses problems with simulating, modeling, and processing. With regard to the simulations, the wafer summary, a portion of which is shown in table 15 , determines which chips are "acceptable." The I-V characteristics and the ring oscillator plots of these chips verify that the parameter values are realistic. For the unacceptable chips, this information along with the parameter values help determine if the I-V data, SUXES, or SPICE need reexamination. Also, this information helps determine if it is worthwhile modifying the strategy file to make these chips acceptable. With regard to modeling efforts, the parameters at the top of table 12(a), if remodeled properly, would be responsible for decreasing the percent difference between the measured and simulated ring oscillator frequencies. These same parameters, if reoptimized, may be responsible for increasing the number of acceptable chips. With regard to the processing efforts, wafer maps reveal distinctive patterns of nonuniformity in semiconductor processes. A wafer map of the measured frequency and wafer maps of the parameters at the top of table 12(b) may assist to develop processes which minimize nonuniformity. For example, ring oscillator frequency variations may be minimized by providing tighter controls on the polysilicon linewidth variations, which may be due to variations in film thickness [15].

\section{ACKNOWLEDGMENTS}

Many thanks go to J. A. Horst who measured the threshold voltages and I-V data points, developed the Accutest computer software to obtain these measurements, and transferred these data to the VAX. We appreciate the software made available to us by M. Gaitan. For comments on this report, we thank H. S. Bennett, A. R. Hefner, and L. W. Linholm. The editorial assistance of J. A. Gonzalez was invaluable. The help or support of all others involved, including J. Albers and C. L. Wilson, is appreciated. 


\section{REFERENCES}

[1] Doganis, K., and Dutton, R. W., SUXES: Stanford University Extractor of Model Parameters (Users Manual), U.S. Army Research Office Contract No. DAAG-29-80-KC-0013 and DARPA Contract No. MDA903-79-C-0257, Integrated Circuits Laboratory, Stanford University, Stanford, CA (November 1982).

[2] Nagel, L. W., SPICE2: A Computer Program to Simulate Semiconductor Circuits, Memorandum No. ERL-M520, Electronics Research Laboratory, University of California, Berkeley, CA (May 1975).

[3] Mattis, R. L., Semiconductor Measurement Technology: A FORTRAN Program for Analysis of Data from Microelectronic Test Structures, NBS Special Publication 40075 (July 1983); Mattis, R. L., and Zucker, R., Release Notes for STAT2 Version 1.31: An Addendum to NBS Special Publication 400-75, NBSIR 83-2779 (November 1983); Mattis, R. L., Release Notes for STAT2 Version 1.7: An Addendum to NBS Special Publication 400-75, NBSIR 86-3333 (March 1986).

[4] Nye, B., Sangiovanni-Vincentelli, A., Spoto, J., and Tits, A., DELIGHT.SPICE: An Optimization-Based System for the Design of Integrated Circuits, Proc. 1983 IEEE Custom Integrated Circuits Conf., pp. 233-238, May 1983.

[5] Scharfetter, D. L., Tremain Jr., R. E., Oki, T. J., Doganis, K., and Chen, S., An Integrated IC Process Characterization Facility, IEDM Tech. Dig., 246-249 (December 1983).

[6] Vladimirescu, A., and Liu, S., The Simulation of MOS Integrated Circuits Using SPICE2, Memorandum No. ERL-M80/7, Electronics Research Laboratory, University of California, Berkeley, CA (February 1980).

[7] Alexander, D. R., Antinone, R. J., and Brown, G. W., SPICE2 MOS Modeling Handbook, BDM/A-77-071-TR, The BDM Corporation, Albuquerque, NM (May 1977).

[8] Cassard, J. M., A Sensitivity Analysis of SPICE Parameters Using an Eleven-Stage Ring Oscillator, IEEE Trans. Electron Devices ED - 31 (2), 264-269 (1984) and IEEE J. Solid State Circuits SC-19 (1), 130-135 (February 1984).

[9] Unit Software \& Consulting Inc., SPICE2 Error Messages and Explanations, (November 1982).

[10] Snedecor, G. W., and Cochran, W. G., Statistical Methods, (The Iowa State University Press, Ames, IA, 1967), pp. 172-198.

[11] Mendenhall, W., Introduction to Probability and Statistics, (Duxbury Press, Belmont, CA, 1971), pp. 276-279.

[12] Buehler, M. G., Grant, S. D., and Thurber, W. R., Bridge and van der Pauw Sheet Resistors for Characterizing the Linewidth of Conducting Layers, J. Electrochem. 
Soc. 125 (4), 650-654 (April 1978).

[13] Buehler, M. G., and Linholm, L. W., Role of Test Chips in Coordinating Logic and Circuit Design and Layout Aids for VLSI, Solid State Technology 24 (9), 68-74 (September 1981).

[14] Sasaki, N., Higher Harmonic Generation in CMOS/SOS Ring Oscillators, IEEE Trans. Electron Devices ED - 29 (2), 280-283 (February 1982).

[15] Perloff, D. S., Wahl, F. E., and Reimer, J. D., Contour Maps Reveal Non-Uniformity In Semiconductor Processing, Solid State Technology 20 (2), 31-36, 42 (February 1977).

[16] Doganis, K., and Scharfetter, D. L., General Optimization and Extraction of IC Device Model Parameters, IEEE Trans. Electron Devices ED - 30 (9), 1219-1228 (September 1983). 


\section{Appendix A - The Program Listing for the Computer Procedure KEYS}

This appendix contains the computer procedure KEYS. It is ordered by file type. The four different file-types specified are command procedures (with a .COM or .XXX extension), FORTRAN files (with a .FOR extension), data files (with a .FIL, .DAT, or .XXX extension), and editor files (with a .WAY extension). They are listed in this order and the files within each file-type are listed in the order of their occurrence. The command procedures are given first and they are:

(1) KEYS.COM

(2) LAST3.COM

(3) INKEY.XXX

(4) SUXES.XXX

(5) SPICEIV.XXX

(6) RMSKEY.COM

(7) STKEY.COM

(8) CORKEY.XXX

(9) MAPKEY.XXX

The FORTRAN files included in this Appendix are listed second and they are:

(1) RCKEY.FOR

(2) SUKEY.FOR

(3) CAPKEY.FOR

(4) IVKEY.FOR

(5) SUMKEY.FOR

(6) RANKEY.FOR

The data files are listed third and they are:

(1) NLW.FIL

(2) RC.FIL

(3) LINE.FIL

(4) LOTNUMBER.FIL 
(5) NPARIN.FIL

(6) PPARIN.FIL

(7) NINPUT.XXX

(8) PINPUT.XXX

(9) NOPT.FIL

(10) POPT.FIL

(11) NSTRAT.FIL

(12) PSTRAT.FIL

(13) IVCHARN.XXX

(14) IVCHARP.XXX

(15) NMODEL.XXX

(16) PMODEL.XXX

(17) SUBCKT.XXX

(18) CIRCUIT.FIL

(19) PCHIPS.FIL

(20) STEND.FIL

(21) COREF.FIL

The editor files that are listed fourth are:

(1) EDNO.WAY

(2) EDSUXN.WAY

(3) EDSUXP.WAY

(4) EDPAR.WAY

(5) EDSPICEN.WAY

(6) EDSPICEP.WAY

(7) EDLAST.WAY

(8) EDNRMS.WAY

(9) EDPRMS.WAY 
(10) EDRES.WAY

(11) EDSKEY.WAY

(12) EDMAP.WAY

(13) EDSIMF.WAY

(14) EDPDIFF.WAY

(15) EDMEAF.WAY

(16) EDMAPS.WAY

Refer to table C-4 for a more complete listing of the file names. 


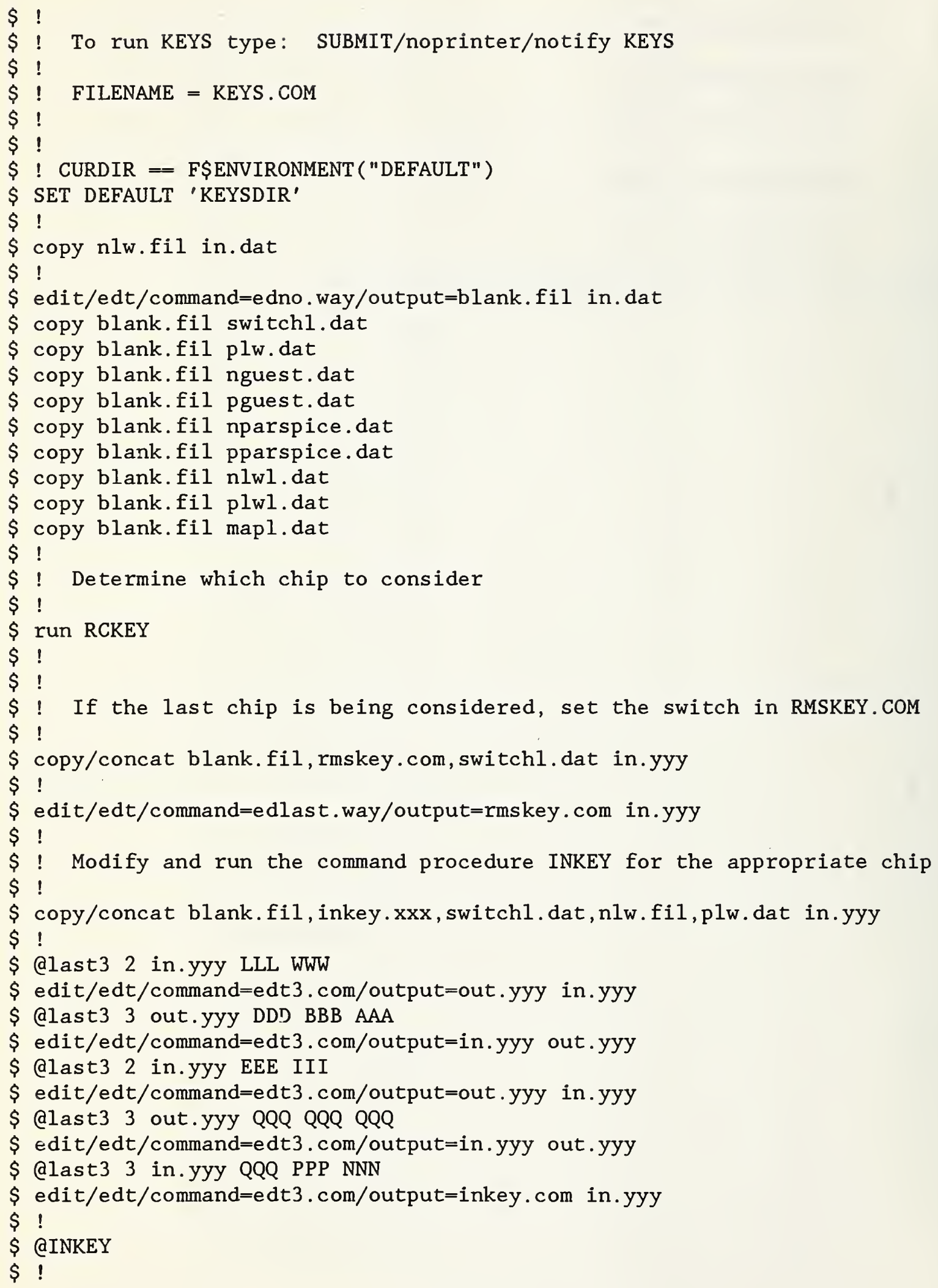


\$ ! Procedure LAST3.COM - - Reads the last 1, 2 or 3 records of a file

$\$$ ! and generates an EDT command file which makes substitutions based

$\$$ ! on the contents of those last three records. P1 contains the

$\$$ ! number 1,2 , or 3 and indicates how many substitute commands are

$\$$ ! to be produced. P2 contains the name of the file to be edited.

\$! The output file is always EDT3.COM. P3 contains the string which

$\$$ ! is to be replaced by the string in the last line. P4 contains the

\$! string which is to be replaced by the next to the last line. P5

$\$$ ! contains the string which is to be replaced by the second to last

$\$$ ! line.

$\$ !$

$\$ !$ On any of the last three lines which are processed, if there are any

$\$$ ! leading spaces, these spaces are removed and the text on that line

$\$ !$ is left justified in the EDT3.COM file.

$\$$ !

\$S :== "WRITE SYS\$OUTPUT "

\$ IF (P1.EQ.1) .OR. (P1.EQ.2) .OR. (P1.EQ.3) THEN GOTO LO

\$ WS "Argument missing or not in range 1 to 3 "

\$ EXIT

$\$ !$

\$ LO:

\$ OPEN/READ INFILE 'P2'

$\$ !$

\$ Branch depending on how many records are to be examined.

$\$ !$

\$ IF P1.EQ.1 THEN GOTO L1

\$ IF P1.EQ.2 THEN GOTO L2

\$ IF P1.EQ.3 THEN GOTO L3

$\$ !$

\$! Case 1: Generate EDT3.COM based on just the last record in the file.

$\$ !$

\$ L1:

\$READ/END_OF_FILE=TOO_FEW INFILE RECORD1

\$ L11:

\$ READ/END_OF_FILE=L12 INFILE RECORD1

\$ GOTO L11

$\$$ !

\$ L12:

$\$$ CLOSE INFILE

\$ OPEN/WRITE OUTFILE EDT3.COM

\$!

$\$$ ! Left justify the last record.

$\$ !$

\$ LENGTH := 'F\$LENGTH(RECORD1)'

$\$$ IF LENGTH .LE. 1 THEN GOTO L14

$\$$ FIRST_SPACE := 'F\$LOCATE(" ",RECORD1)'

$\$$ IF FIRST_SPACE .NE. O THEN GOTO L14

$\$$ RECORD1 $:=$ 'F\$EXTRACT (1, LENGTH, RECORD1)'

$\$$ ! 


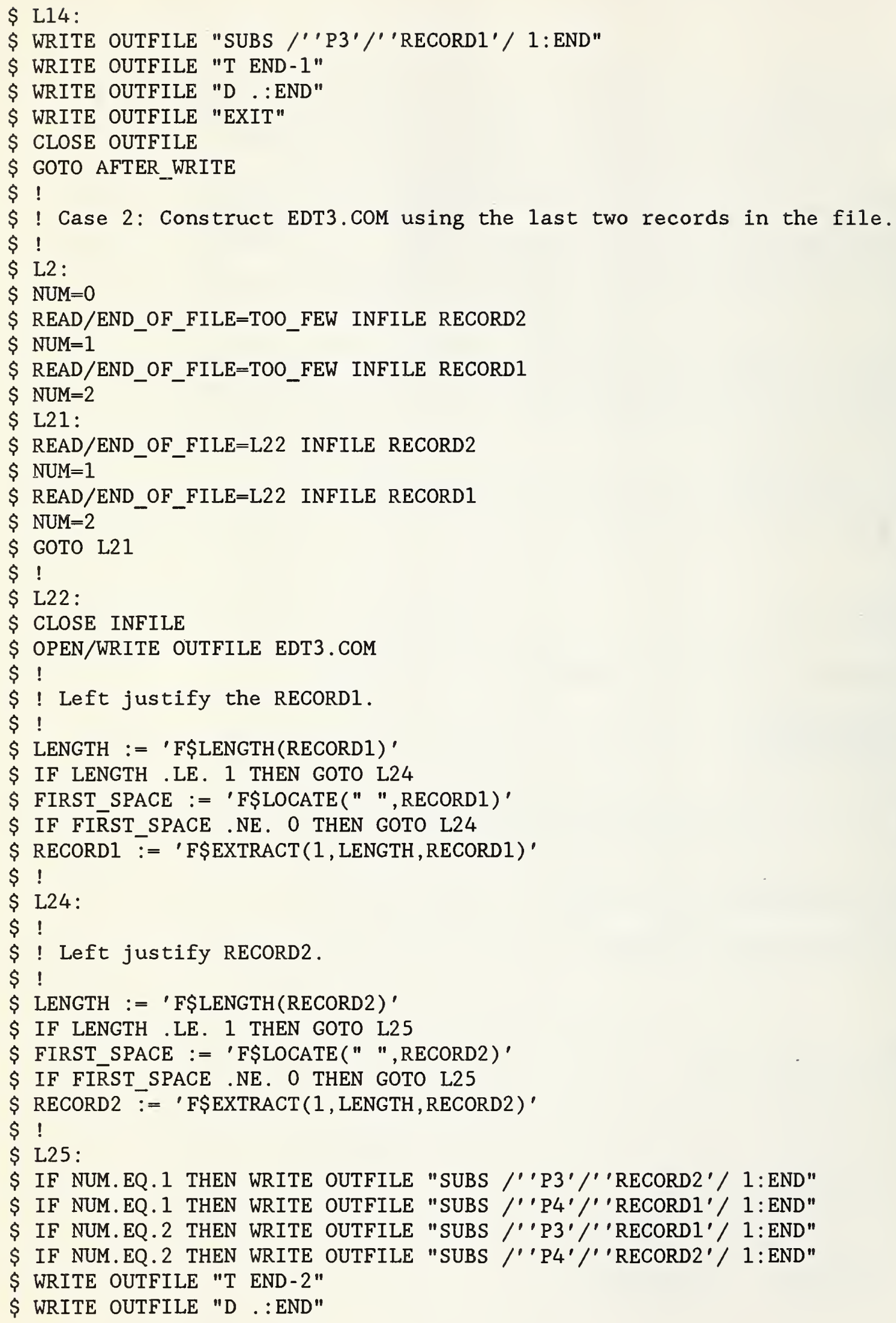


$\$$ WRITE OUTFILE "EXIT"

$\$$ CLOSE OUTFILE

\$ GOTO AFTER_WRITE

$\$ !$

\$ L3:

$\$ !$

\$ Case 3: Construct EDT3.COM using last 3 records of file.

$\$ !$

$\$ N U M=0$

\$ READ/END_OF_FILE=TOO_FEW INFILE RECORD3

$\$ N U M=1$

\$ READ/END_OF_FILE=TOO_FEW INFILE RECORD2

$\$ N U M=2$

\$READ/END_OF_FILE=TOO_FEW INFILE RECORD1

$\$ N U M=3$

\$ L31:

\$ READ/END_OF_FILE=L32 INFILE RECORD3

$\$ N U M=1$

\$ READ/END_OF_FILE=L32 INFILE RECORD2

$\$ N U M=2$

$\$$ READ/END_OF_FILE=L32 INFILE RECORD1

$\$ N U M=3$

\$ GOTO L31

$\$$ !

\$ L32:

$\$$ CLOSE INFILE

$\$$ OPEN/WRITE OUTFILE EDT3.COM

$\$ !$

$\$$ ! Left justify RECORD1.

$\$ !$

$\$$ LENGTH := 'F\$LENGTH(RECORD1)'

$\$$ IF LENGTH. LE. 1 THEN GOTO L33

\$FIRST_SPACE := 'F\$LOCATE(" ",RECORD1)'

$\$$ IF FIRTST_SPACE .NE. O THEN GOTO L33

$\$$ RECORD1 $:=$ 'F\$EXTRACT $(1$, LENGTH, RECORD1)'

$\$ !$

L33:

$\$$ !

$\$$ ! Left justify RECORD2.

$\$ !$

\$ LENGTH := 'F\$LENGTH(RECORD2)'

$\$$ IF LENGTH . LE. 1 THEN GOTO L34

$\$$ FIRST_SPACE := 'F\$LOCATE(" ",RECORD2)'

\$ IF FIRST_SPACE .NE. O THEN GOTO L34

$\$$ RECORD2 $:=$ 'F\$EXTRACT (1, LENGTH, RECORD2)'

$\$$ !

\$ L34:

$\$ !$

\$ ! Left justify RECORD3.

$\$ !$

\$ LENGTH := 'F\$LENGTH(RECORD3)'

$\$$ IF LENGTH .LE. 1 THEN GOTO L35 


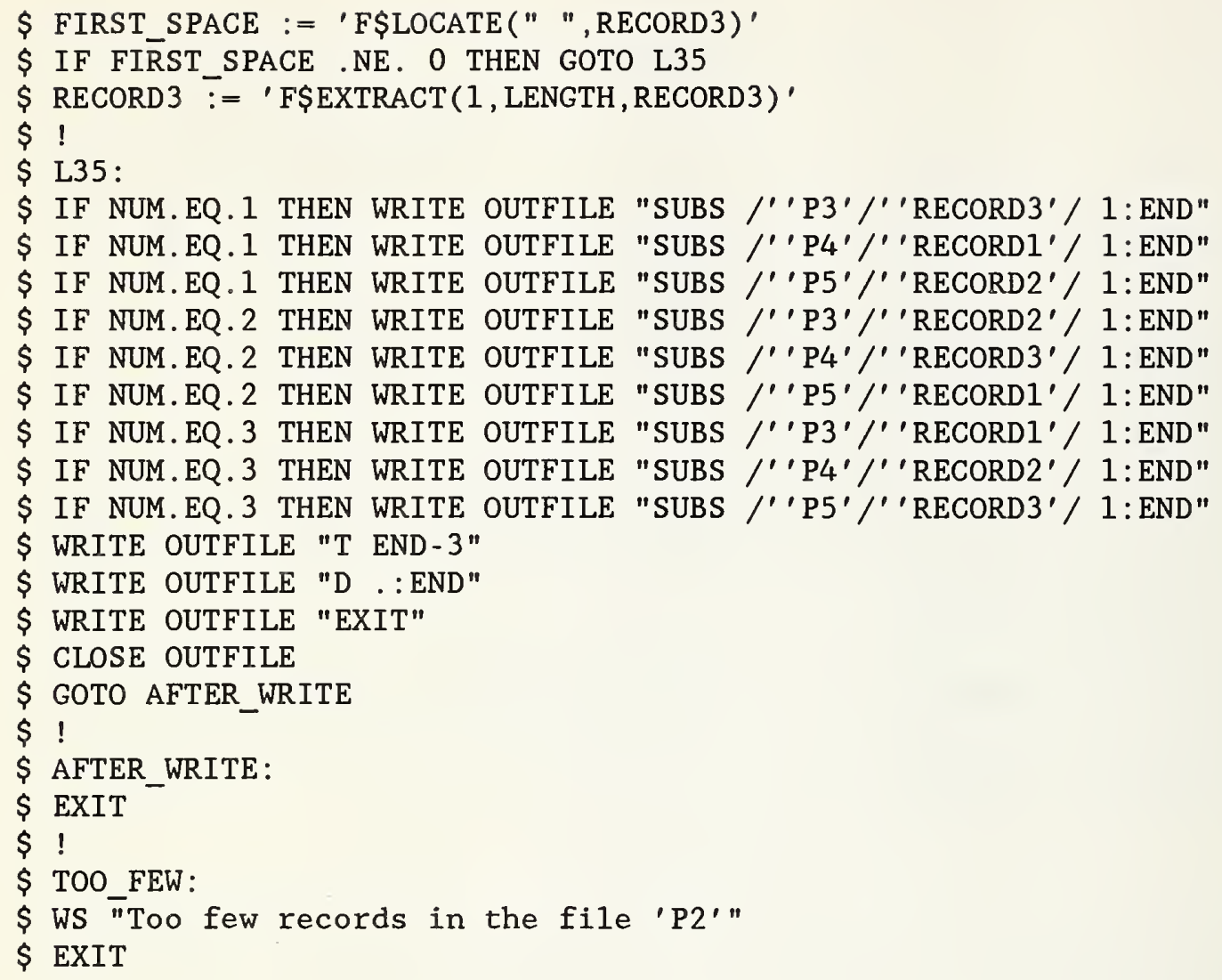




\section{INKEY.XXX}

$\$ !$

FILENAME: INKEY.COM

In the program SUKEY, the width is asked for first then the length.

\$ SET DEFAULT 'KEYSDIR'

\$ copy WAAARBBBCDDDVB.DAT vbin, dat

$\$$ ! Consider the n-channel MOSFETs.

$\$$ !

NNN

$\$$ IF L . EQS. O THEN GOTO PCHAN

\$ copy nWAAARBBBCDDD. dat in. dat

$\$$ copy nparin.fil in3. dat

$\$$ !

\$! Gather the initial estimates for SUXES.

$\$ !$

$\$$ run SUKEY

\section{III}

EEE

$\$$ copy guest.dat nguest.dat

$\$$ copy out. dat nWAAARBBBCDDDF. dat

\$ copy out2. dat nWAAARBBBCDDDS . dat

$\$$ !

$\$ !$ Insert the parameters in the SUXES input file.

$\$ !$

$\$$ copy/concat blank.fil, ninput.xxx, nguest.dat in.yyy

$\$ !$

\$ Qlast3 3 in. yyy VVV VVV VVV

$\$$ edit/edt/command=edt 3 . com/output=out. yyy in. yyy

\$ Qlast 3 out.yyy VVV VVV VVV

$\$$ edit/edt/command=edt 3 .com/output=in. yyy out. yyy

\$ @last3 2 in. yyy VVV VVV

$\$$ edit/edt/command=edt 3 . com/output=out. yyy in. yyy

$\$$ Qlast 32 out. yyy HHH VVV

\$ edit/edt/command=edt 3. com/output=in. yyy out. yyy

$\$$ Qlast3 2 in.yyy KKK VVV

$\$$ edit/edt/command=edt 3 . com/output=out. yyy in. yyy

$\$$ Qlast3 2 out.yyy JJJ VVV

\$ edit/edt/command=edt 3 .com/output=in. yyy out.yyy

\$ @last3 2 in.yyy MMM VVV

$\$$ edit/edt/command=edt 3 . com/output=out . yyy in . yyy

$\$$ Qlast 32 out. yyy $000 \mathrm{VVV}$

$\$$ edit/edt/command=edt 3 . com/output=in. yyy out. yyy

\$ @last3 2 in. yyy RRR VVV

$\$$ edit/edt/command=edt 3 . com/output=out. yyy in. yyy

$\$$ Qlast3 2 out.yyy SSS VVV 
$\$$ edit/edt/command=edt3. com/output=in. yyy out. yyy

\$ @last3 2 in.yyy TTT VVV

$\$$ edit/edt/command=edt 3 .com/output=out. yyy in.yyy

$\$$ @last3 2 out.yyy UUU VVV

$\$$ edit/edt/command=edt 3. com/output=ninput.dat out.yyy

$\$ !$

$\$$ ! Modify and run the command procedure for suXES for the appropriate

$\$ ! \quad$ chip and device type.

$\$ !$

$\$$ copy/concat blank.fil, suxes.xxx,plw.dat in.yyy

$\$ !$

\$ @last3 2 in.yyy VVV VVV

$\$$ edit/edt/command=edt3.com/output=out. yyy in.yyy

\$ @last3 3 out.yyy ggg aza ccc

$\$$ edit/edt/command=edt3.com/output=in. yyy out. yyy

$\$$ edit/edt/command=edsuxn.way/output=suxes.com in.yyy

$\$$ !

\$ copy ninput.dat input.dat

$\$$ copy nopt.fil opt.dat

$\$$ copy nstrat.fil strat.dat

$\$$ !

\$ @SUXEs

$\$$ !

$\$$ ! Gather the extracted parameters.

$\$ !$

\$ copy expar.dat in.dat

$\$$ !

$\$$ edit/edt/command=edpar.way/output=exparn.dat in.dat

$\$$ !

$\$$ copy exparn.dat expar.dat

$\$$ run CAPKEY

$\$ !$

$\$$ copy parspice.dat nparspice.dat

$\$ !$

\$! Insert the width and length into the SPICE file which obtains the

$\$ ! \quad$ IV characteristics.

$\$ !$

$\$$ copy/concat blank.fil, ivcharn. $x x x, n l w . f i l$ in.yyy

$\$ !$

\$ @last3 2 in.yyy BZB FFF

$\$$ edit/edt/command=edt 3 . com/output=out. yyy in. yyy

\$ @last3 3 out.yyy VVV VVV VVV

$\$$ edit/edt/command=edt 3 . com/output=ivchar.fil out.yyy

$\$$ !

$\$$ copy ivchar.fil ivcharn.fil

$\$ !$

$\$$ ! Put together the n-channel MOSFET models file for SPICE.

$\$ !$

$\$$ copy/concat blank.fil, nmodel.xxx, nparspice.dat in.yyy

\$!

$\$$ alast3 3 in. yyy vvv aqa bqb

$\$$ edit/edt/command=edt 3 . com/output=out. yyy in. yyy 
\$ @last 33 out.yyy cqc dqd eqe

$\$$ edit/edt/command=edt3. com/output=in. yyy out. yyy

$\$$ Qlast3 3 in.yyy fqf gqg hqh

$\$$ edit/edt/command=edt3. com/output=out. yyy in.yyy

\$ @last3 3 out.yyy iqi jqj kqk

$\$$ edit/edt/command=edt 3 . com/output=in. yyy out. yyy

$\$$ Qlast3 3 in.yyy lql mqm nqn

$\$$ edit/edt/command=edt 3 . com/output=out. yyy in. yyy

\$ Qlast3 3 out.yyy oqo pqp rqr

$\$$ edit/edt/command=edt 3. com/output=in. yyy out. yyy

$\$$ @last 31 in. yyy sqs

$\$$ edit/edt/command=edt 3 .com/output=nmodel.fil in.yyy

$\$$ !

\$ COPY/CONCAT BLANK.FIL, NMODEL.FIL, IVCHARN.FIL IVCHARN.CEL

$\$$ copy ivcharn.cel ivchar.cel

$\$ !$

$\$$ ! Modify the command procedure which obtains the IV characteristics.

$\$ !$

\$ copy/concat blank. fil, spiceiv.xxx, nlwl.dat in.yyy

\$ edit/edt/command=edspicen.way/output=out.yyy in.yyy

$\$$ Qlast3 2 out.yyy gzg fzf

$\$$ edit/edt/command=edt 3. com/output=in. yyy out.yyy

$\$$ Qlast3 3 in.yyy eze dzd czc

$\$$ edit/edt/command=edt 3 . com/output=spiceiv. com in. yyy

$\$ !$

$\$$ ! Simulate the n-channel MOSFET IV characteristics.

$\$ !$

\$ @SPICEIV

$\$$ !

$\$$

$\$ !$

$\$$ ! Repeat the above for the P-CHANNEL MOSFETs.

$\$ !$

$\$$ PCHAN :

PPP

$\$$ IF M .EQS. 0 THEN GOTO CKT

$\$$ copy pWAAARBBBCDDD.dat in.dat

$\$$ copy pparin.fil in3.dat

$\$ !$

$\$$ ! Gather the initial estimates for sUXES.

$\$ !$

$\$$ run SUKEY

WWW

LLL

$\$$ copy guest.dat pguest.dat

$\$$ copy out.dat pWAAARBBBCDDDF. dat

\$ copy out2. dat pWAAARBBBCDDDS. dat

$\$ !$

$\$$ ! Insert the parameters in the SUXES input file.

$\$ !$

$\$$ copy/concat blank.fil,pinput.xxx,pguest.dat in.yyy

$\$$ ! 


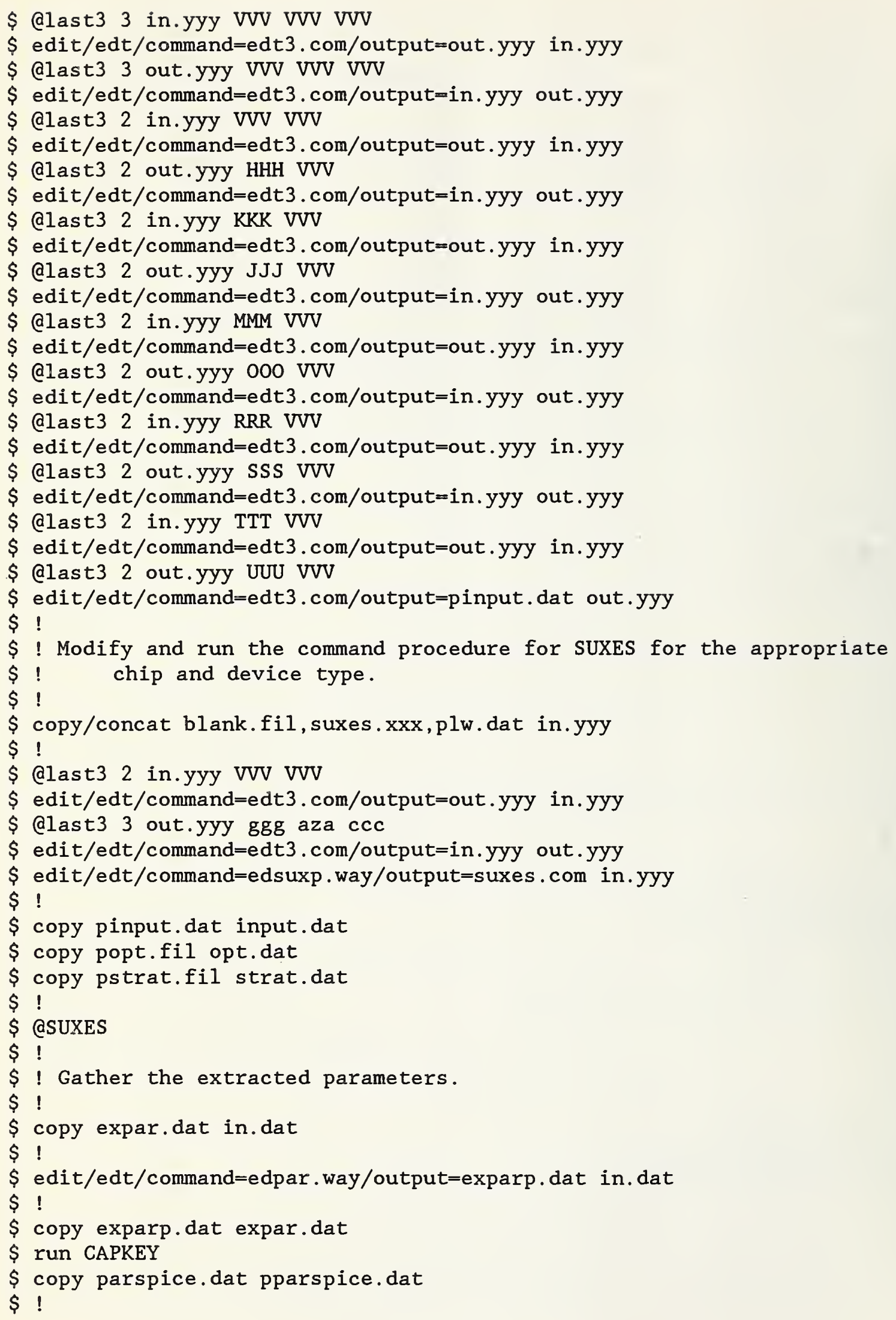


$\$$ ! Insert the width and length into the SPICE file which obtains the

$\$$ copy/concat blank. fil, ivcharp.xxx,plw.dat in.yyy

$\$$ !

\$ @last3 2 in.yyy BZB FFF

$\$$ edit/edt/command=edt 3 .com/output=out.yyy in.yyy

$\$$ Qlast3 3 out.yyy VVV VVV VVV

$\$$ edit/edt/command=edt 3 .com/output=ivchar.fil out.yyy

$\$ !$

$\$$ copy ivchar.fil ivcharp.fil

$\$ !$

$\$$ ! Put together the p-channel MOSFET models file for SPICE.

$\$ !$

$\$$ copy/concat blank.fil,pmodel.xxx,pparspice.dat in.yyy

$\$ !$

\$ @last3 3 in.yyy vvv aqa bqb

\$ edit/edt/command=edt3. com/output=out. yyy in. yyy

\$ Qlast3 3 out.yyy cqc dqd eqe

\$ edit/edt/command=edt3.com/output=in. yyy out. yyy

\$ @last3 3 in.yyy fqf gqg hqh

\$ edit/edt/command=edt3. com/output=out.yyy in.yyy

\$ Qlast3 3 out.yyy iqi jqj kqk

$\$$ edit/edt/command=edt3.com/output=in.yyy out. yyy

\$ Qlast3 3 in.yyy lql mqm nqn

\$ edit/edt/command=edt3.com/output=out. yyy in. yyy

\$ Qlast3 3 out. yyy oqo pqp rqr

$\$$ edit/edt/command=edt 3 . com/output=in. yyy out. yyy

$\$$ Qlast3 1 in.yyy sqs

$\$$ edit/edt/command=edt 3. com/output=pmodel.fil in.yyy

$\$$ !

\$ COPY/CONCAT BLANK. FIL, PMODEL.FIL, IVCHARP.FIL IVCHARP.CEL

$\$$ copy ivcharp.cel ivchar.cel

$\$ !$

$\$$ ! Modify the command procedure which obtains the IV characteristics.

$\$$ !

$\$$ copy/concat blank.fil, spiceiv. $x x x, p l w 1$. dat in.yyy

$\$$ edit/edt/command=edspicep . way/output=out. yyy in. yyy

$\$$ Qlast3 2 out.yyy gzg fzf

$\$$ edit/edt/command=edt 3 . com/output=in. yyy out. yyy

$\$$ Qlast3 3 in.yyy eze dzd czc

$\$$ edit/edt/command=edt 3. com/output=spiceiv.com in. yyy

$\$ !$

$\$$ ! Simulate the p-channel MOSFET IV characteristics.

$\$ !$

$\$$ @SPICEIV

$\$ !$

$\$$

$\$$ !

\$ Simulate the optional dynamic circuit.

$\$ !$

$\$ \mathrm{CKT}$ : 
$\$ !$

$\$$ IF (L .EQS. O) .AND. (M .EQS. 0) THEN GOTO END

\$!

\$!

$\$ !$ Insert the correct widths and lengths into the SPICE file

$\$ !$

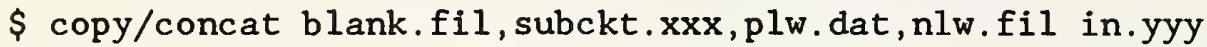

$\$ !$

\$ @last3 2 in.yyy hzh izi

$\$$ edit/edt/command=edt 3 . com/output=out. yyy in. yyy

\$ @last3 3 out.yyy VVV VVV VVV

$\$$ edit/edt/command=edt 3. com/output=in. yyy out. yyy

\$ @last3 2 in.yyy jzj kzk

$\$$ edit/edt/command=edt 3 . com/output=out. yyy in. yyy

\$ Qlast3 3 out.yyy VVV VVV VVV

$\$$ edit/edt/command=edt 3. com/output=subckt.fil out.yyy

$\$ !$

$\$ !$

\$! Simulate the dynamic circuit.

$\$ !$

$\$$ copy/concat -

blank.fil, nmodel.fil,pmodel.fil, subckt.fil, circuit.fil circuit.cel

$\$ !$

$\$$ RUN SPICENBS

CIRCUIT. CEL

CIRCUIT. DAT

CIRCUIT.PLT

$\$$ !

$\$$ ! Plot the results of the dynamic circuit.

$\$ !$

$\$$ ASSIGN CIRCUIT.PLT FOR011

$\$$ RUN S5PLOT

G

WAAARBBBCDDD\$

TIME (

NODE VOLTAGE (V) $\$$

$\$$ RUN MCVAX

DEVICE LPA0: P300

READ IOP020.DAT

PLOT

EXIT

$\$$ ! SET TERM/BRO

\$ END:

$\$ !$

$\$$ ! Sum up the results for this chip.

$\$ !$

\$ submit/noprinter/notify RMSKEY

$\$$ ! 


\section{SUXES . XXX}

$\$ !$

\$! FILENAME: SUXES.COM

$\$ !$

\$! This command procedure runs SUXES which obtains the values for most

$\$$ ! of the SPICE parameters.

$\$ !$

$\$$ purge *.*

\$ RUN SSUXES

6,5

$\mathrm{N}$

$\mathrm{Y}$

1

INPUT

2

77WcccRazaCgggF

77WcccRazaCgggS

OPT

STRAT

$\mathrm{N}$

F

22

$\mathrm{N}$

EXPAR

Q

$\$ !$ 


\section{SPICEIV .XXX}

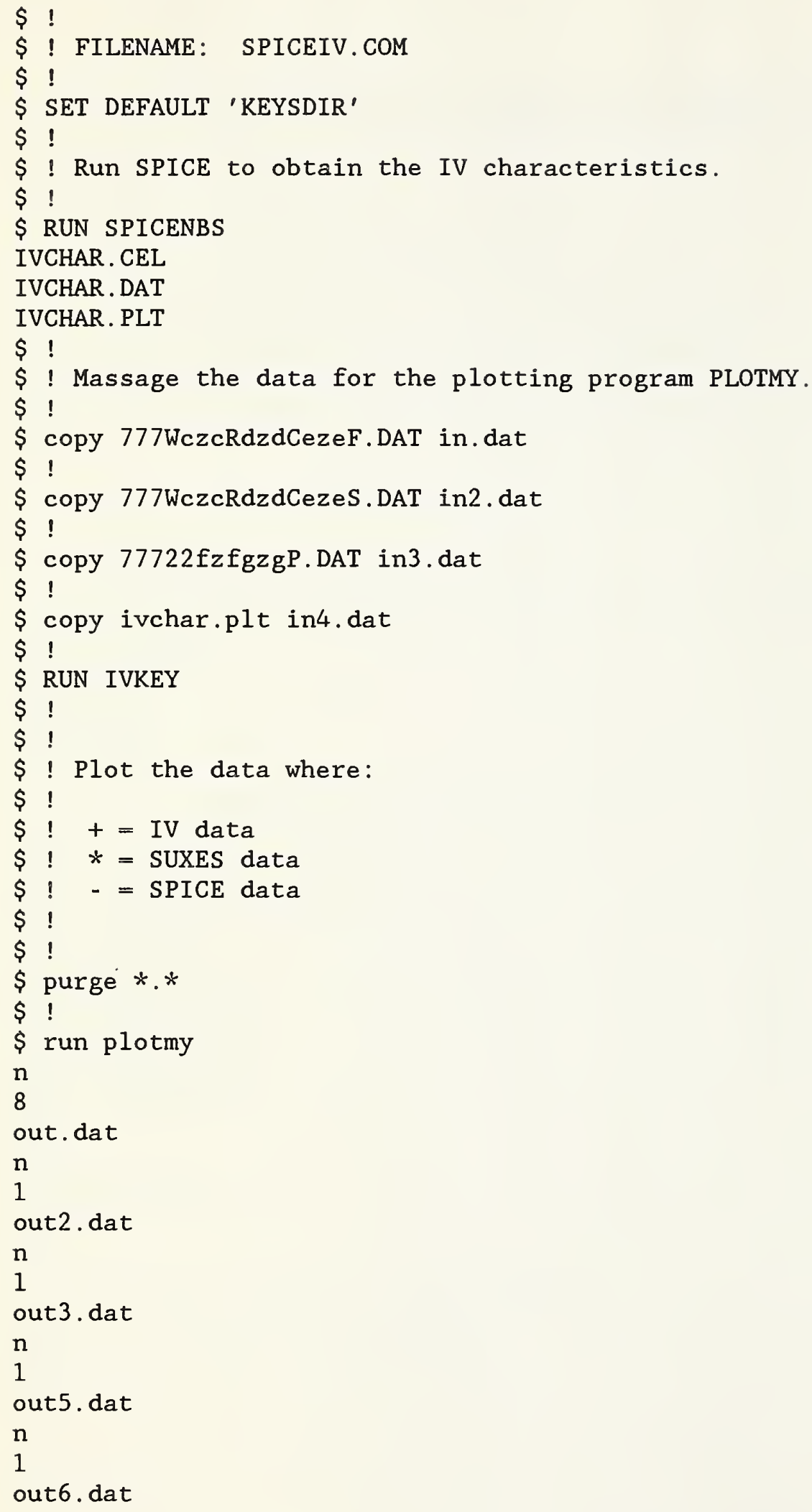


n

1

out7. dat

$\mathrm{n}$

1

out8. dat

n

1

out9. dat

n

1

1

777WczcRdzdCeze\$

VDS (V) $\$$

IDS (A) $\$$

2

ก

n

y

$+$

$+$

*

-

$\$$ RUN MCVAX

DEVICE LPAO: P300

READ IOP020.DAT

PLOT

EXIT

$\$$ ! 


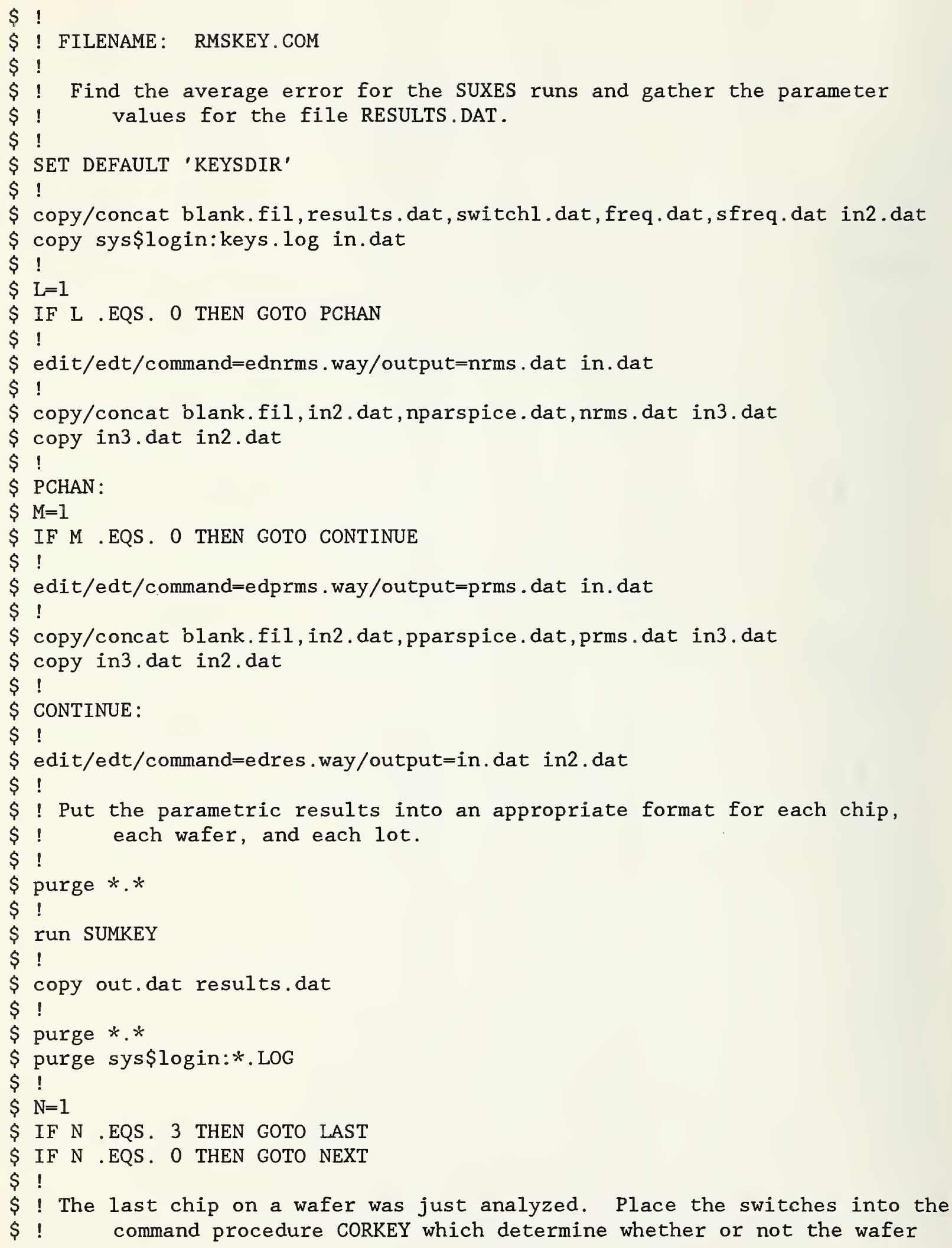




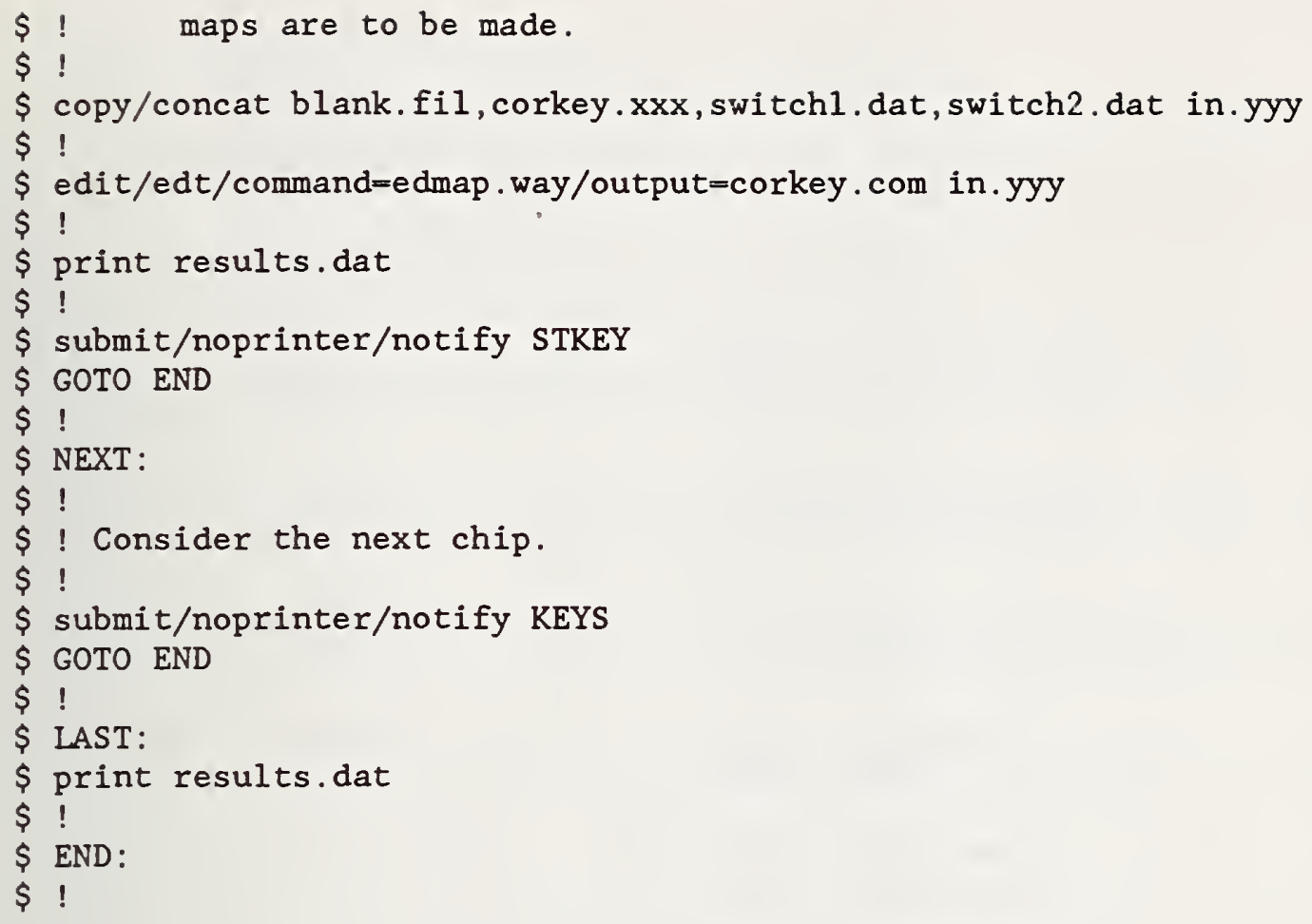




\section{STKEY.COM}

$\$ !$

\$! FILENAME: STKEY.COM

$\$ !$

$\$$ SET DEFAULT 'KEYSDIR'

$\$ !$

\$! Prepare the parametric data files for STAT2.

$\$ !$

$\$$ edit/edt/command=edskey. way/output=in.dat w.dat

$\$$ copy/concat blank.fil, stend.fil, in.dat w.dat

$\$ !$

$\$$ edit/edt/command=edskey. way/output=in.dat 1 . dat

$\$$ copy/concat blank.fil,stend.fil, in.dat 1.dat

$\$$ !

$\$$ edit/edt/command=edskey. way/output=in.dat wp.dat

\$ copy/concat blank.fil,stend.fil, in.dat wp.dat

$\$$ !

$\$$ edit/edt/command=edskey. way/output=in.dat 1p.dat

$\$$ copy/concat blank.fil, stend.fil, in.dat lp.dat

$\$$ !

\$ edit/edt/command=edskey.way/output=in.dat mckt.dat

$\$$ copy/concat blank.fil,stend.fil, in. dat mckt.dat

$\$ !$

$\$$ edit/edt/command=edskey.way/output=in.dat sckt.dat

\$ copy/concat blank.fil, stend.fil,in.dat sckt.dat

$\$ !$

$\$$ edit/edt/command=edskey.way/output=in.dat pdiff.dat

$\$$ copy/concat blank.fil, stend.fil, in.dat pdiff.dat

$\$$ !

$\$ !$

$\$$ edit/edt/command=edskey.way/output=in. dat ncgdo.dat

\$ copy/concat blank. fil, stend.fil, in.dat ncgdo.dat

$\$ !$

$\$$ edit/edt/command=edskey.way/output=in.dat ncgbo.dat

\$ copy/concat blank. fil,stend.fil, in.dat ncgbo.dat

$\$ !$

$\$$ edit/edt/command=edskey. way/output=in.dat nrms.dat

\$ copy/concat blank.fil, stend.fil, in.dat nrms.dat

$\$ !$

$\$$ edit/edt/command=edskey. way/output=in. dat nld.dat

$\$$ copy/concat blank.fil,stend.fil, in.dat nld.dat

$\$ !$

$\$$ edit/edt/command=edskey. way/output=in.dat ntox.dat

$\$$ copy/concat blank. fil, stend.fil, in.dat ntox.dat

$\$$ !

$\$$ edit/edt/command=edskey. way/output=in.dat nsub.dat

$\$$ copy/concat blank.fil, stend.fil,in.dat nsub.dat

$\$$ !

$\$$ edit/edt/command=edskey. way/output=in.dat nvto.dat

$\$$ copy/concat blank.fil, stend.fil, in.dat nvto.dat

$\$$ ! 
$\$$ edit/edt/command=edskey.way/output=in.dat nkp.dat

\$ copy/concat blank. fil, stend.fil, in.dat nkp.dat

$\$$ !

\$ edit/edt/command=edskey.way/output=in.dat ngamma.dat

\$ copy/concat blank.fil, stend.fil, in.dat ngamma.dat

$\$$ !

$\$$ edit/edt/command=edskey.way/output=in. dat nphi.dat

$\$$ copy/concat blank.fil, stend.fil, in.dat nphi.dat

$\$$ !

$\$$ edit/edt/command=edskey.way/output=in.dat nu0.dat

$\$$ copy/concat blank. fil, stend.fil, in. dat nu0. dat

$\$$ !

$\$$ edit/edt/command=edskey.way/output=in.dat ndelta.dat

$\$$ copy/concat blank. fil, stend.fil, in.dat ndelta.dat

$\$$ !

$\$$ edit/edt/command=edskey. way/output=in. dat nvmax.dat

\$ copy/concat blank. fil, stend.fil, in. dat nvmax.dat

$\$$ !

$\$$ edit/edt/command=edskey.way/output=in.dat nxj.dat

$\$$ copy/concat blank. fil, stend.fil, in.dat $n \times j$.dat

$\$$ !

$\$$ edit/edt/command=edskey.way/output=in.dat nkappa.dat

$\$$ copy/concat blank. fil, stend.fil, in. dat nkappa.dat

$\$$ !

\$ edit/edt/command=edskey. way/output=in. dat nnfs.dat

$\$$ copy/concat blank. fil, stend.fil, in.dat nnfs.dat

$\$$ !

\$ edit/edt/command=edskey. way/output=in. dat neta.dat

$\$$ copy/concat blank.fil, stend.fil, in.dat neta.dat

$\$$ !

\$ edit/edt/command=edskey. way/output=in.dat ntheta.dat

$\$$ copy/concat blank. fil, stend.fil, in.dat ntheta.dat

$\$$ !

\$ edit/edt/command=edskey. way/output=in.dat nerr.dat

$\$$ copy/concat blank.fil, stend.fil,in.dat nerr.dat

$\$$ !

$\$$ purge *.*

$\$$ !

$\$$ edit/edt/command=edskey. way/output=in.dat pcgdo.dat

$\$$ copy/concat blank. fil, stend.fil,in.dat pcgdo.dat

$\$$ !

$\$$ edit/edt/command=edskey. way/output=in.dat pcgbo.dat

\$ copy/concat blank. fil, stend.fil, in.dat pcgbo.dat

$\$$ !

\$ edit/edt/command=edskey. way/output=in.dat prms.dat

$\$$ copy/concat blank.fil, stend.fil, in.dat prms.dat

$\$$ !

$\$$ edit/edt/command=edskey. way/output=in.dat pld.dat

\$ copy/concat blank.fil,stend.fil,in.dat pld.dat

$\$$ !

$\$$ edit/edt/command=edskey. way/output=in.dat ptox.dat

$\$$ copy/concat blank.fil, stend.fil, in.dat ptox.dat 
$\$ !$

$\$$ edit/edt/command=edskey. way/output=in.dat psub.dat

$\$$ copy/concat blank.fil,stend.fil,in.dat psub.dat

$\$ !$

$\$$ edit/edt/command=edskey. way/output=in.dat pvto.dat

$\$$ copy/concat blank.fil,stend.fil,in.dat pvto.dat

$\$ !$

$\$$ edit/edt/command=edskey. way/output=in.dat pkp.dat

$\$$ copy/concat blank.fil,stend.fil,in.dat pkp.dat

$\$ !$

$\$$ edit/edt/command=edskey. way/output=in.dat pgamma.dat

$\$$ copy/concat blank.fil, stend.fil, in.dat pgamma.dat

$\$ !$

$\$$ edit/edt/command=edskey. way/output=in.dat pphi.dat

\$ copy/concat blank.fil,stend.fil,in.dat pphi.dat

$\$ !$

$\$$ edit/edt/command=edskey.way/output=in.dat pu0.dat

$\$$ copy/concat blank.fil,stend.fil,in.dat pu0.dat

$\$ !$

$\$$ edit/edt/command=edskey.way/output=in.dat pdelta.dat

$\$$ copy/concat blank.fil,stend.fil, in.dat pdelta.dat

$\$ !$

$\$$ edit/edt/command=edskey. way/output=in.dat pvmax.dat

$\$$ copy/concat blank.fil, stend.fil, in.dat pvmax.dat

$\$ !$

$\$$ edit/edt/command=edskey.way/output=in.dat $\mathrm{pxj}$.dat

$\$$ copy/concat blank.fil,stend.fil,in.dat pxj.dat

$\$ !$

$\$$ edit/edt/command=edskey.way/output=in.dat pkappa.dat

\$ copy/concat blank.fil,stend.fil, in.dat pkappa.dat

$\$ !$

$\$$ edit/edt/command=edskey.way/output=in.dat pnfs.dat

$\$$ copy/concat blank.fil,stend.fil,in.dat pnfs.dat

$\$ !$

$\$$ edit/edt/command=edskey.way/output=in.dat peta.dat

$\$$ copy/concat blank.fil,stend.fil, in.dat peta.dat

$\$ !$

$\$$ edit/edt/command=edskey.way/output=in.dat ptheta.dat

$\$$ copy/concat blank.fil,stend.fil, in.dat ptheta.dat

$\$ !$

\$ edit/edt/command=edskey. way/output=in.dat perr.dat

$\$$ copy/concat blank.fil, stend.fil, in.dat perr.dat

$\$ !$

$\$$ purge $* . *$

$\$ !$

$\$$ ! Create the database for STAT2.

$\$ !$

$\$$ run $c r d b$

SUXES'S VALUES

4

$\$ !$

$\$ !$ Run STAT2 to fill the database and obtain the correlation coefficients. 


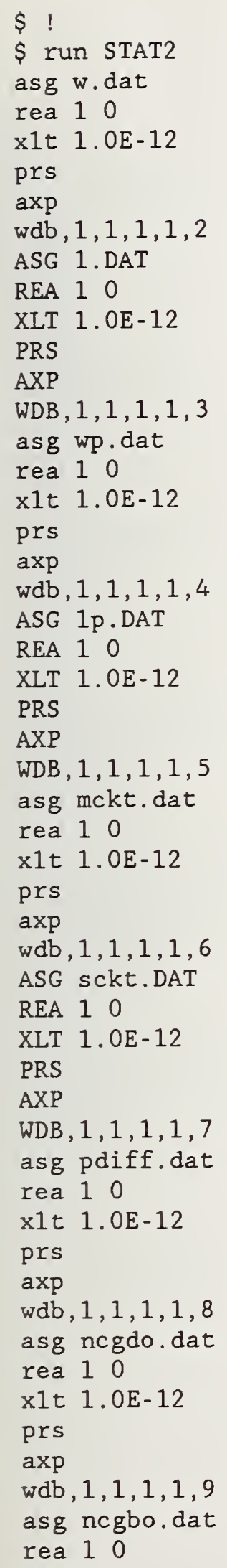




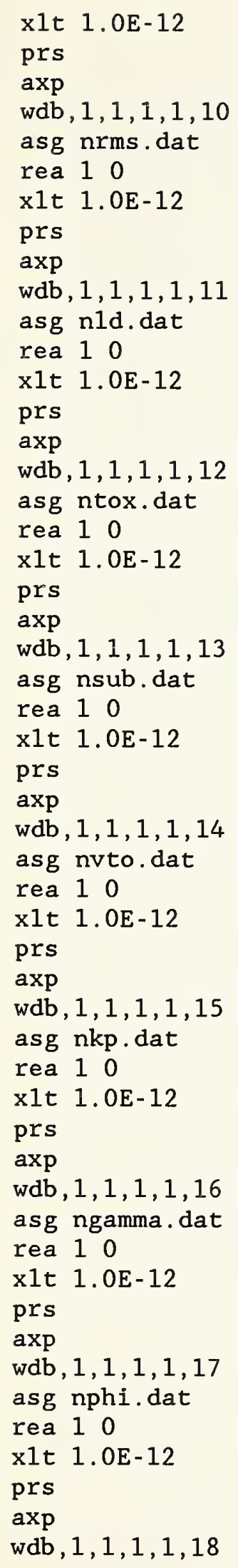




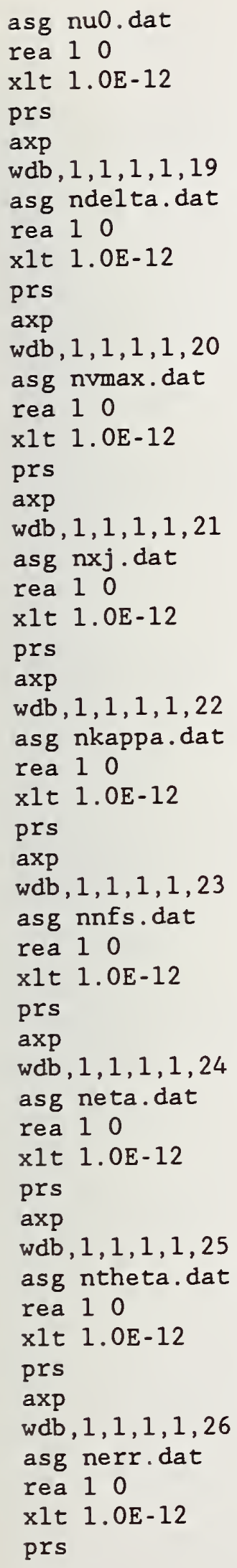




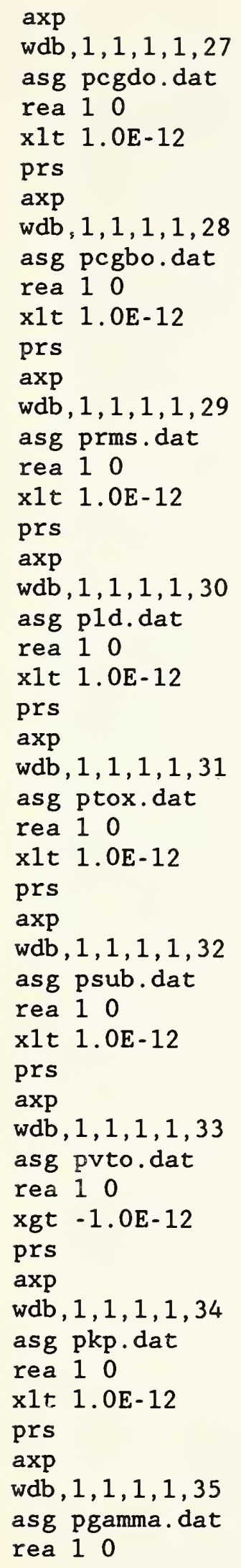




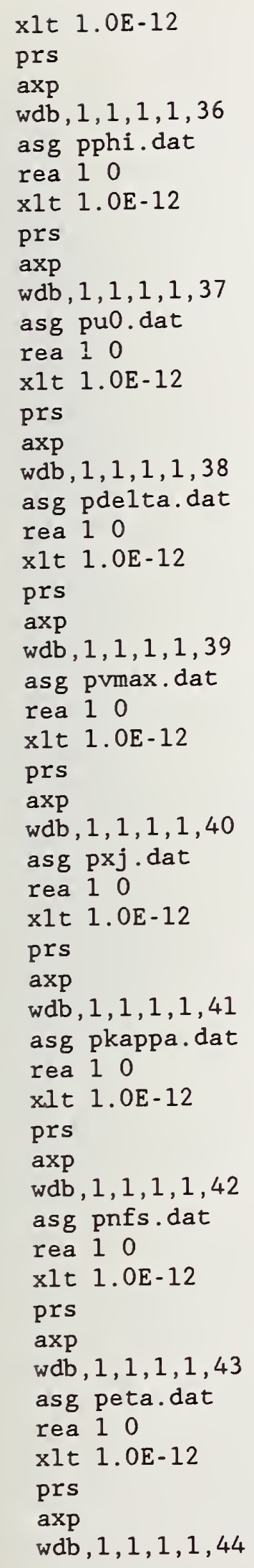




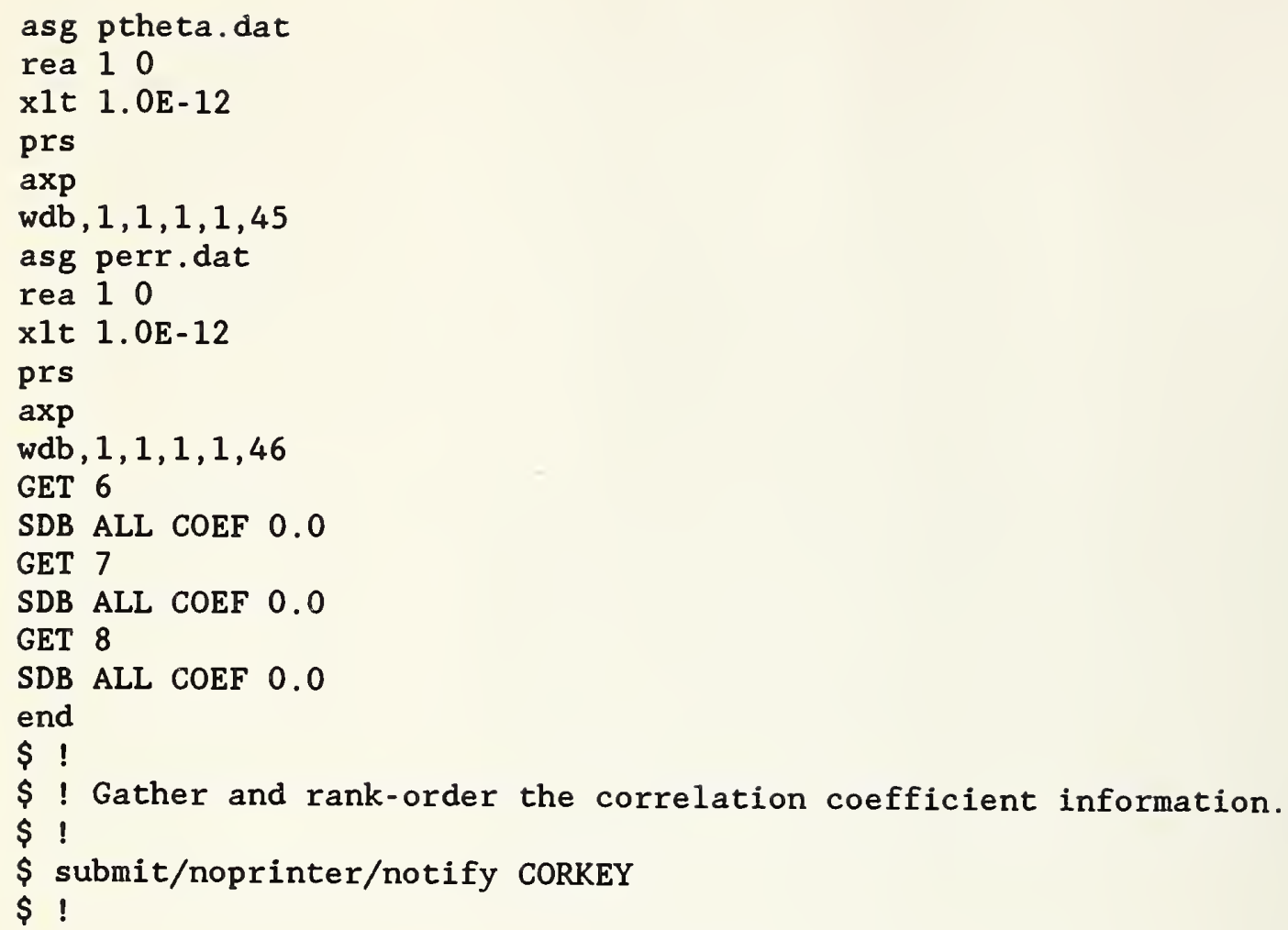




\section{CORKEY.XXX}

$\$ !$

\$! FILENAME: CORKEY.COM

$\$ !$

\$ SET DEFAULT 'KEYSDIR'

$\$ !$

\$ copy sys\$login:stkey.log in2.dat

$\$ !$

\$! Gather and rank-order the correlation coefficients with respect to the

$\$$ ! simulated frequency.

$\$ !$

$\$$ edit/edt/command=edsimf.way/output=in.dat in2.dat

$\$$ !

$\$$ run RANKEY

$\$$ print out.dat

$\$ !$

\$! Gather and rank-order the correlation coefficients with respect to the

$\$ !$ percent difference between the measured and simulated frequencies.

$\$ !$

$\$$ edit/edt/command=edpdiff. way/output=in.dat in2.dat

$\$ !$

$\$$ run RANKEY

$\$$ copy out2. dat mapl.dat

$\$$ print out.dat

$\$$ !

\$! Gather and rank-order the correlation coefficients with respect to the

$\$ !$ measured frequency.

$\$ !$

$\$$ edit/edt/command=edmeaf. way/output=in. dat in2. dat

$\$ !$

$\$$ rUn RANKEY

$\$$ copy out2. dat map2. dat

$\$$ ! copy out.dat save.dat

$\$$ print out.dat

$\$$ !

$\$ \mathrm{~K}=1$

$\$ N=2$

$\$$ IF $\mathrm{K}$. EQS. 0 THEN GOTO MID

$\$ !$

$\$$ ! Modify the command procedure for MAPKEY to include the appropriate lot

$\$$ copy/concat blank. fil, mapkey.xxx, lotnumber.fil, nlw. fil in.zzz

$\$$ copy/concat blank.fil, in.zzz, map2.dat, mapl.dat, switchl.dat in.yyy

$\$ !$

$\$$ edit/edt/command=edmaps . way/output=out.yyy in. yyy

$\$$ Qlast 3 out.yyy EEE DDD CCC

$\$$ edit/edt/command=edt3.com/output=in. yyy out.yyy

\$ Qlast3 3 in. yyy BBB QQQ QQQ

$\$$ edit/edt/command=edt3. com/output=out. yyy in. yyy

\$ Qlast3 3 out. yyy QQQ QQQ ZZZ 
\$ edit/edt/command=edt3. com/output=in. yyy out. yyy

$\$$ @last3 1 in. yyy AAA

$\$$ edit/edt/command=edt 3 . com/output=mapkey.com in.yyy

$\$$ !

\$ submit/noprinter/notify MAPKEY

\$ GOTO END

$\$ !$

\$ MID:

\$ IF N .EQS. 2 THEN GOTO FINISH

$\$ !$

$\$ !$ Consider the first chip on the next wafer.

$\$ !$

\$ submit/noprinter/notify KEYS

\$ GOTO END

$\$$ !

\$ FINISH:

$\$$ copy results.dat oresults.dat

$\$$ END: 


\section{MAPKEY.XXX}

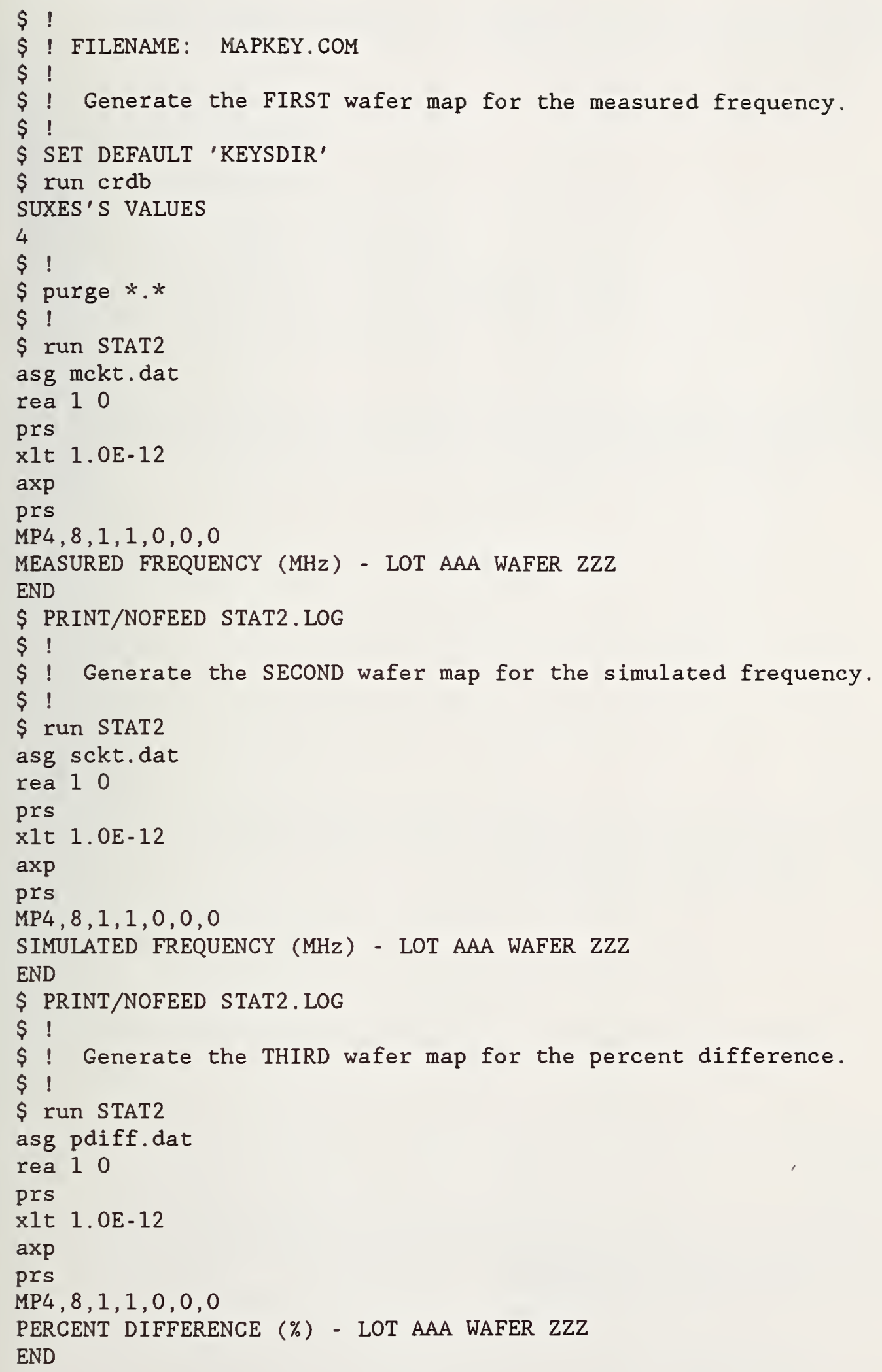




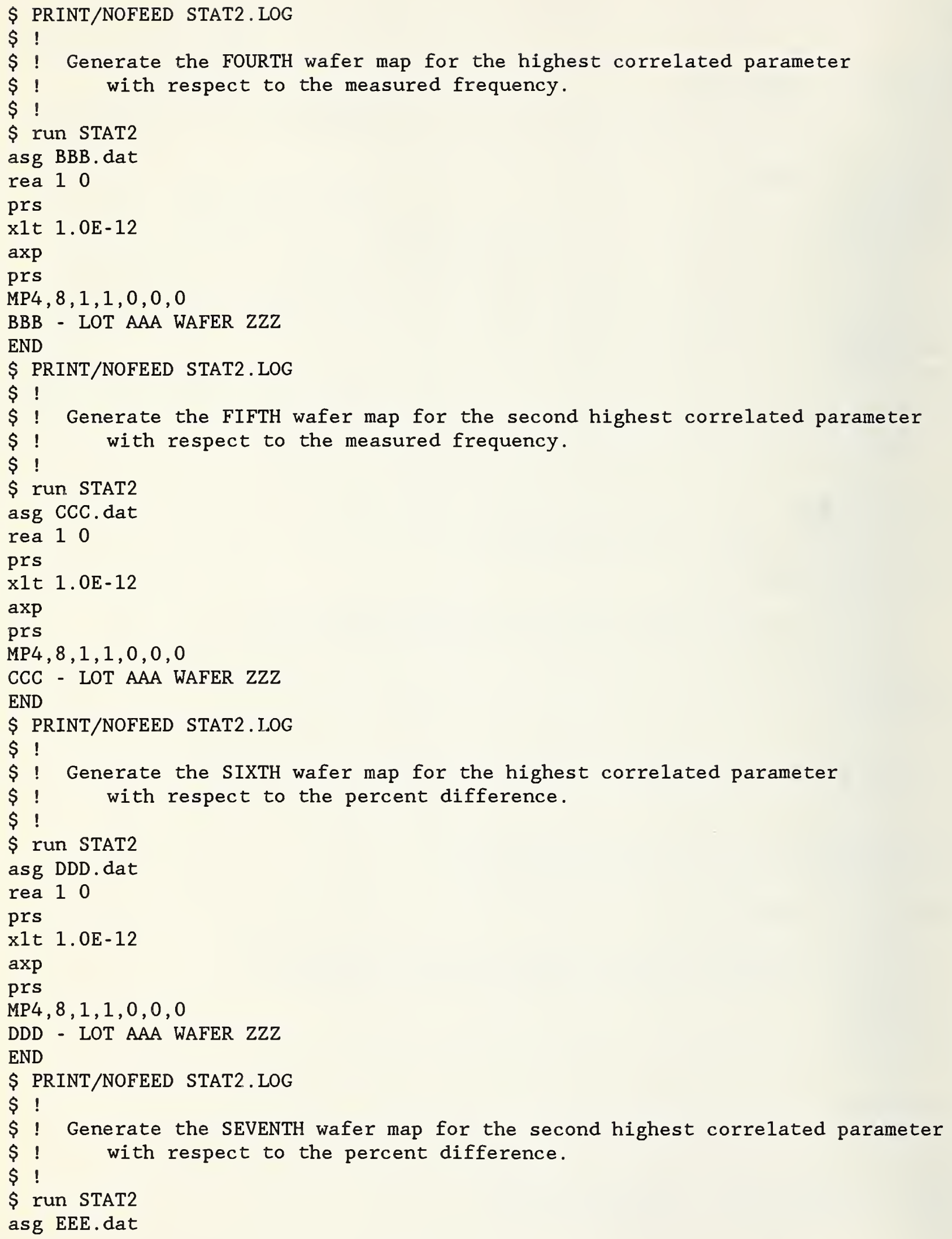




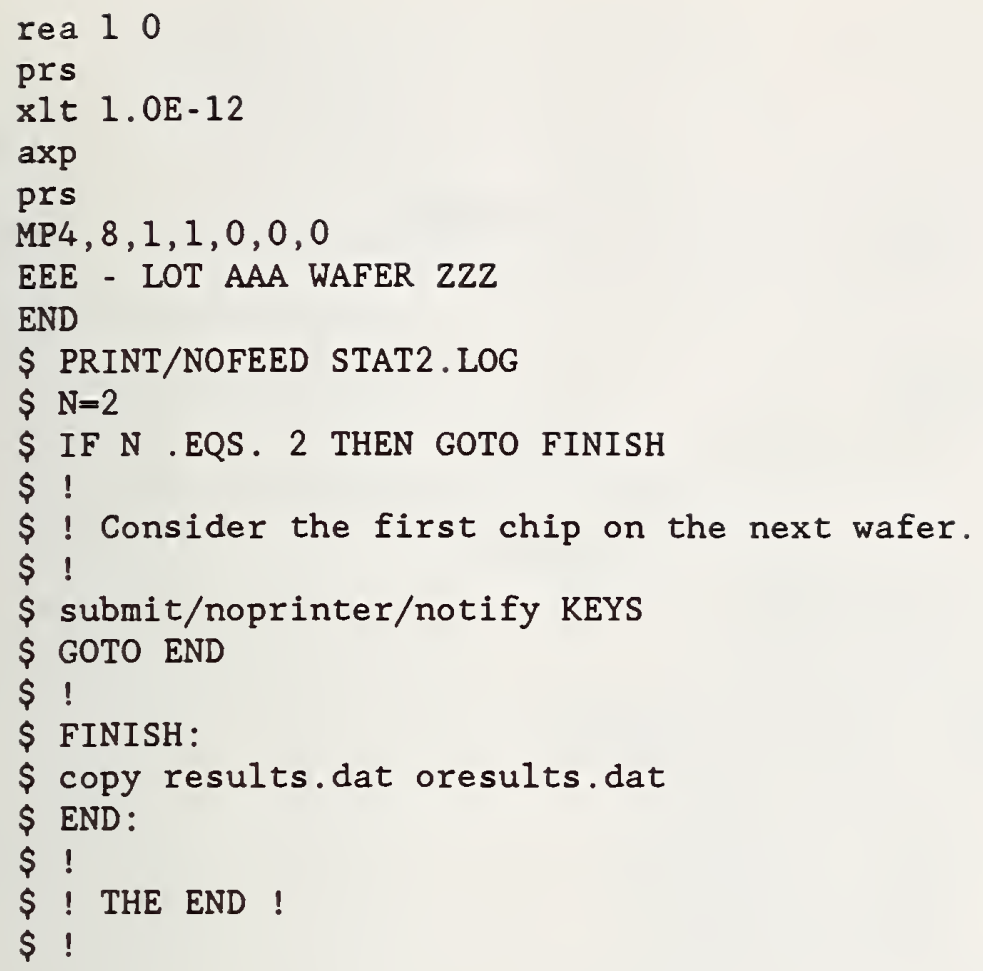




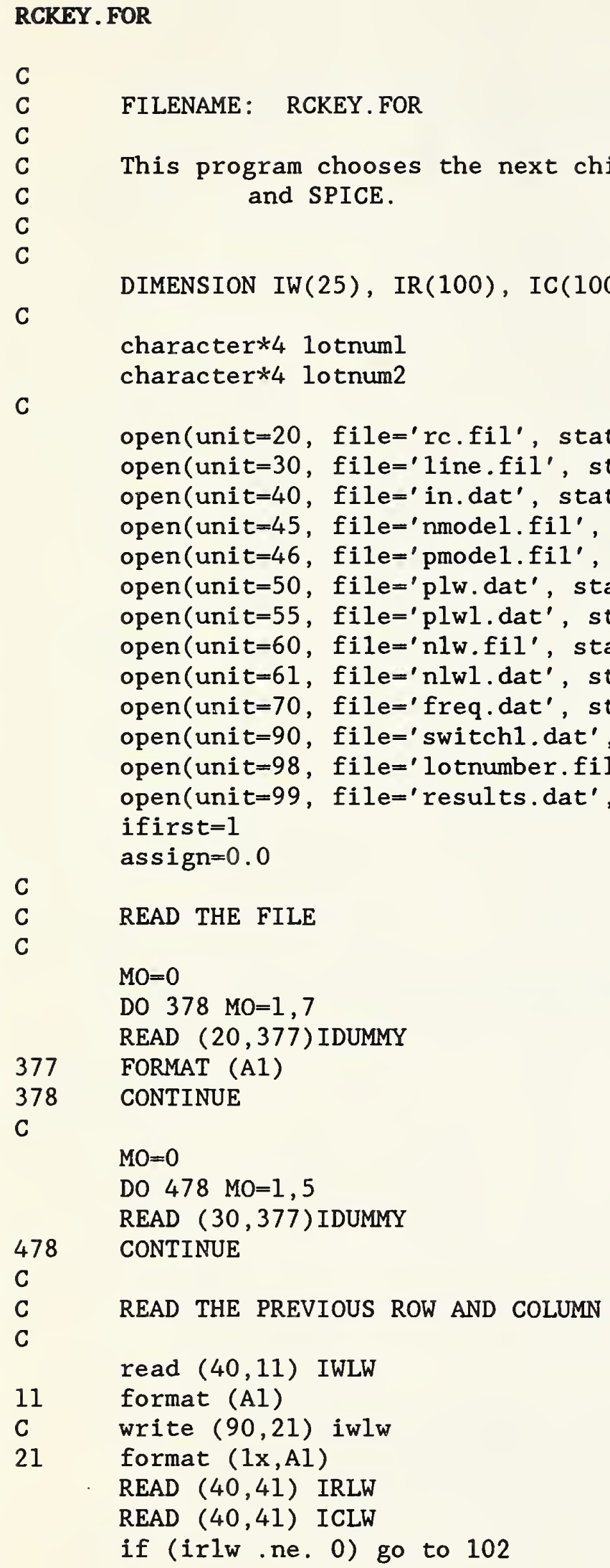

C

C

C

C

C

C

C

C

C

C READ THE FILE

C

377

378

C 


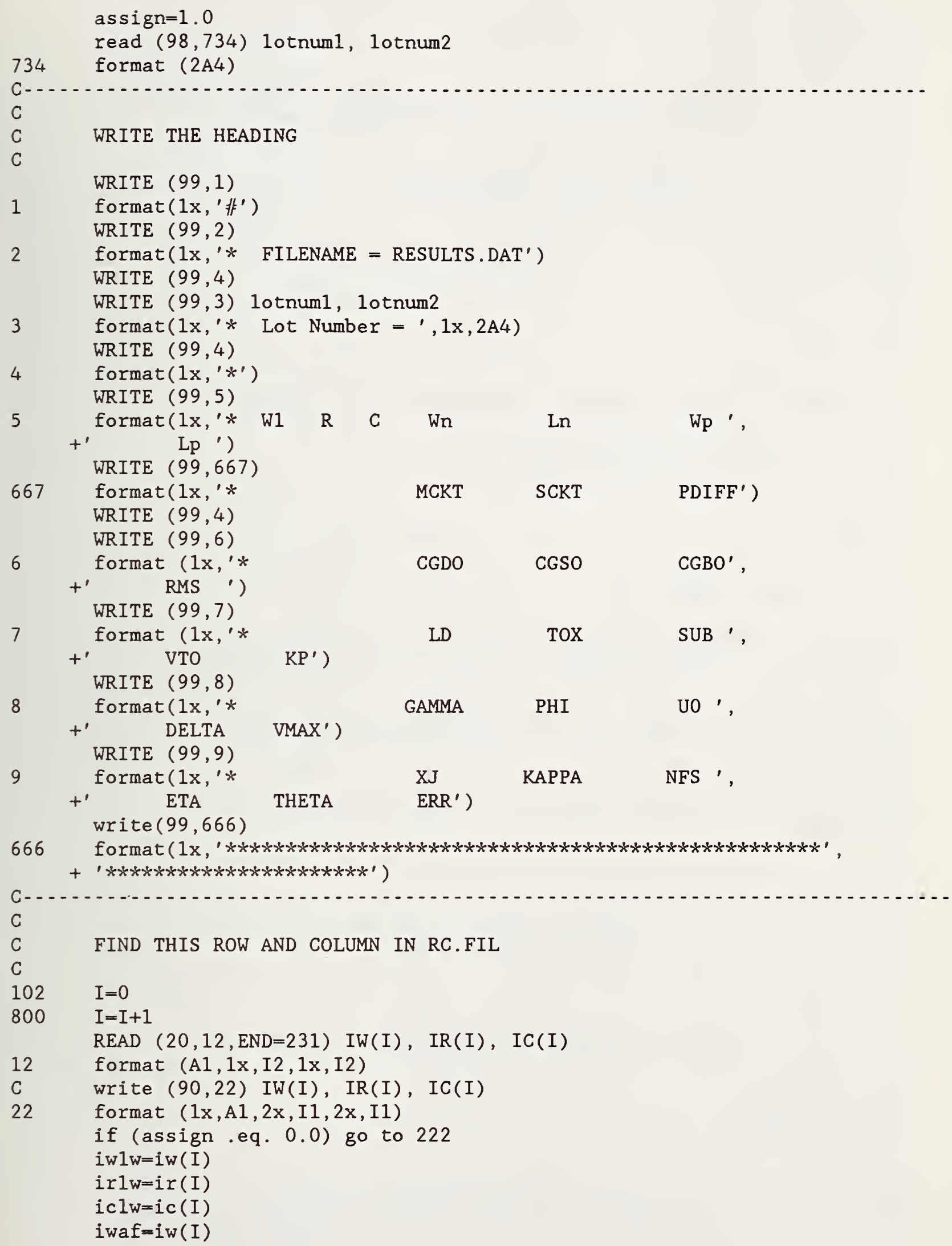




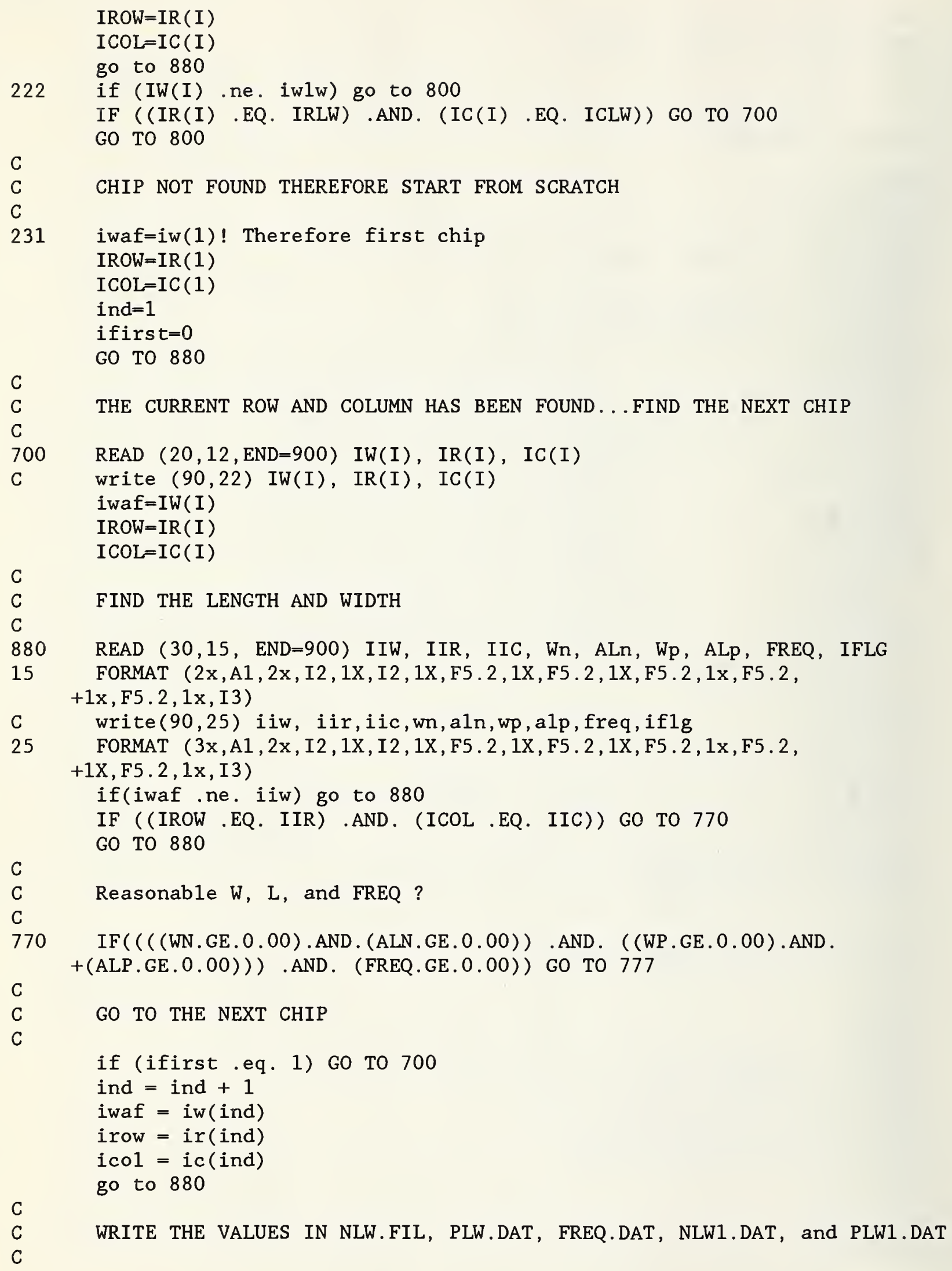

C

C

C 
777 write $(60,10)$ IWAF

WRITE $(60,41)$ IROW

WRITE $(60,41)$ ICOL

WRITE $(60,51)$ WN

WRITE $(60,51)$ ALN

write $(50,10)$ iwaf

WRITE $(50,41)$ IROW

WRITE $(50,41)$ ICOL

WRITE $(50,51)$ WP

WRITE $(50,51)$ ALP

write $(70,10)$ iwaf

WRITE $(70,41)$ IROW

WRITE $(70,41)$ ICOL

WRITE $(70,51)$ WN

WRITE $(70,51)$ ALN

WRITE $(70,51)$ WP

WRITE $(70,51)$ ALP

WRITE $(70,51)$ FREQ

C

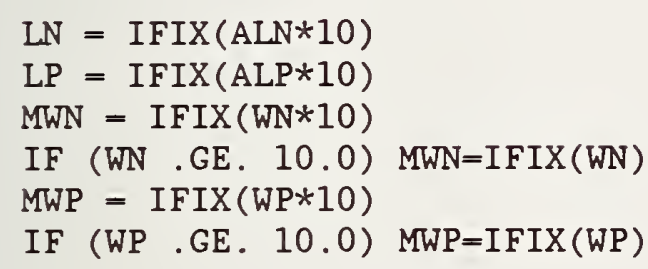

C

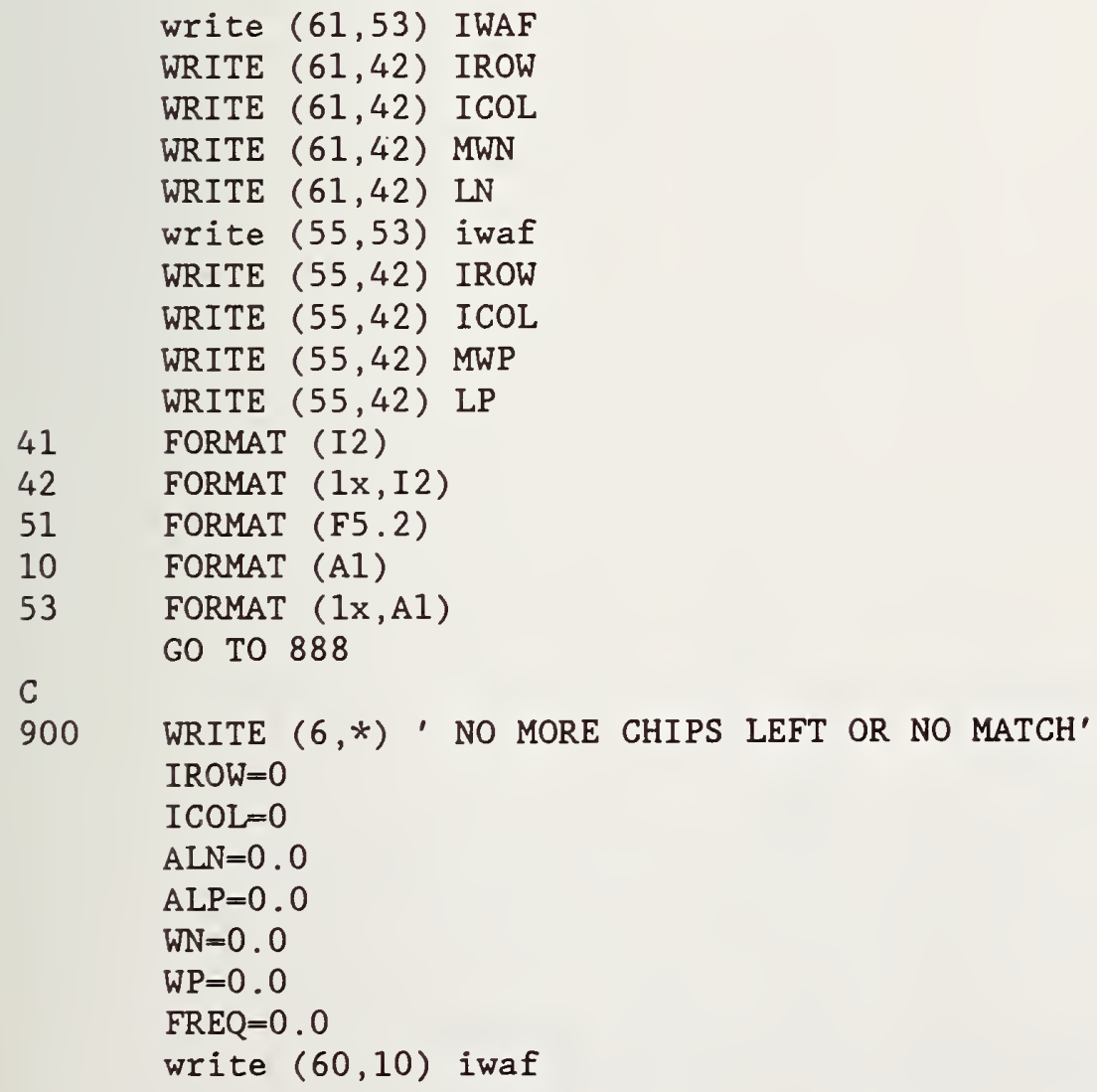

900 WRITE $(6, *)$ ' NO MORE CHIPS LEFT OR NO MATCH' IROW $=0$

$\mathrm{ICOL}=0$

$A L N=0.0$

$A L P=0.0$

$W N=0.0$

$W P=0.0$

FREQ $=0.0$

write $(60,10)$ iwaf

C 


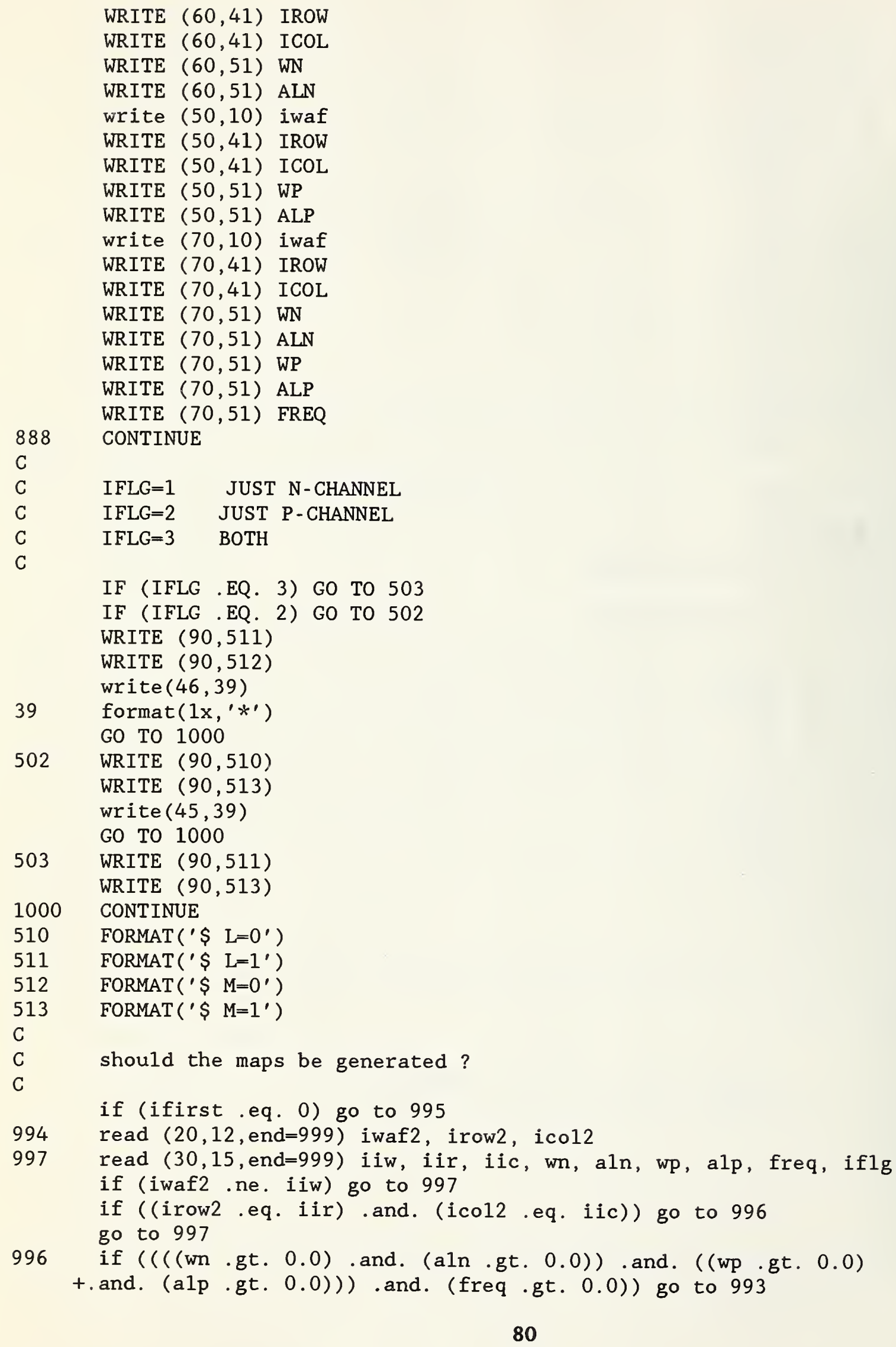


go to 994

C

993 if(iwaf2 .ne. iwaf) go to 992

995 write $(90,61)$ ! Same wafer...

61 format (' $\$ \mathrm{~N}=0^{\prime}$ )

go to 998

C

992 write $(90,81)$ ! Different wafer...

81 format (' $\$ \mathrm{~N}=1^{\prime}$ )

go to 998

C

999 if (assign .eq. 1.0) write $(90,72)$

72 format (' $\left.\$ \mathrm{~N}=3^{\prime}\right)$ ! One chip...

if (assign .eq. 1.0) go to 998

C

write $(90,71) !$ End of lot...

71 format (' $\$ \mathrm{~N}=2^{\prime}$ )

C

998 continue

END 
SUKEY. FOR

C

C

C

C

C

C

C

FILENAME: SUKEY. FOR

This program takes the IV data from the ACCUTEST and makes it suitable for the SUXES program.

DIMENSION VDS(1000), VGS(1000), VBS(1000), CIDS(1000)

DIMENSION X1(100), Y1(100), VBSZ(100)

DIMENSION VDSBE(1000), $\operatorname{VGSBE}(1000), \operatorname{CIDSBE}(1000)$

real $\mathrm{x}(250), \mathrm{y}(250)$, vds $2(500,50)$, beta, vt

C integer $\mathrm{n} 2(50), \mathrm{np}(50)$

C

open (unit $=20$, file='in.dat', status='old')

open (unit=31, file='vbin.dat', status='old')

open (unit $=32, \mathrm{file}==^{\prime}$ in 3 .dat', status='old')

open (unit $=40, f i l e=$ 'out.dat', status='unknown')

open (unit $=50$, file='out2.dat', status='unknown')

open(unit $=80$, file='guest.dat', status='unknown')

open (unit $=90$, file='vbout.dat', status='unknown')

C
$\mathrm{ES}=103.5 \mathrm{E}-12$
$\mathrm{EOX}=34.5 \mathrm{E}-12$
! in $\mathrm{F} / \mathrm{m}$
$\mathrm{Q}=1.602 \mathrm{E}-19$
! in $\mathrm{F} / \mathrm{m}$
! in $\mathrm{C}$

$\mathrm{u} 0=0.0$

$\mathrm{xkp}=0.0$

$v$ to $=0.0$

$\mathrm{MO}=0$

DO $178 \mathrm{MO}=1,16$

READ $(20,177)$ IDUMMY

177 FORMAT (A1)

178 CONTINUE

C

WRITE $(40,2)$

WRITE $(50,2)$

2 FORMAT (' $x$ ', $5 \mathrm{x}$, 'Data from the ACCUTEST')

write $(40,3)$

write $(50,3)$

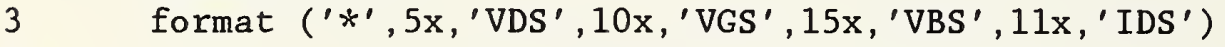

C

write $(6,4)$

4 format (' Width $=$ ')

$\operatorname{read}(5, *) \mathrm{W}$

C

write $(6,5)$

5 format (' Length $=$ ')

$\operatorname{read}(5, *) \mathrm{AL}$

C

write $(40,6) \mathrm{W}, \mathrm{AL}$ 


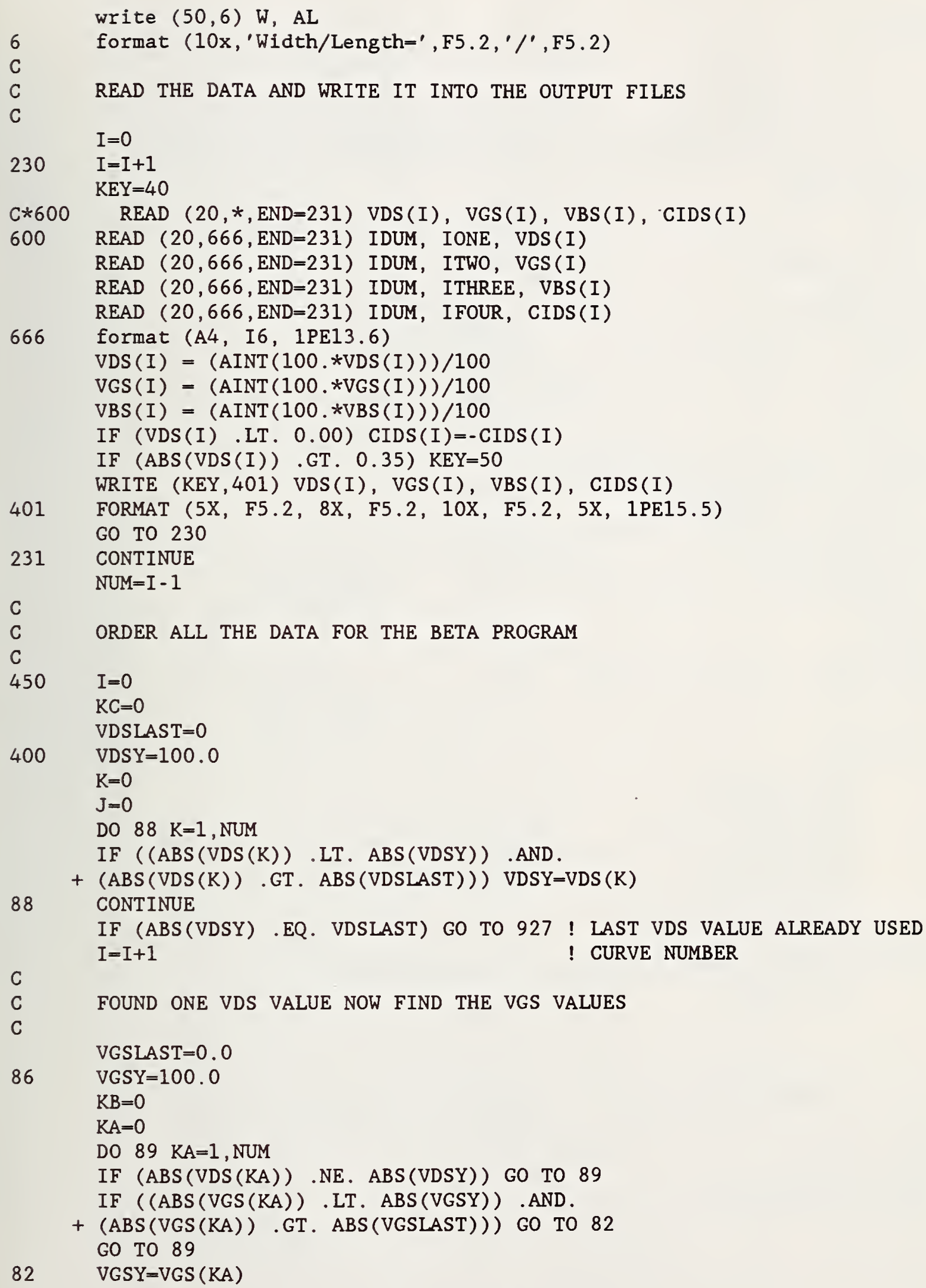




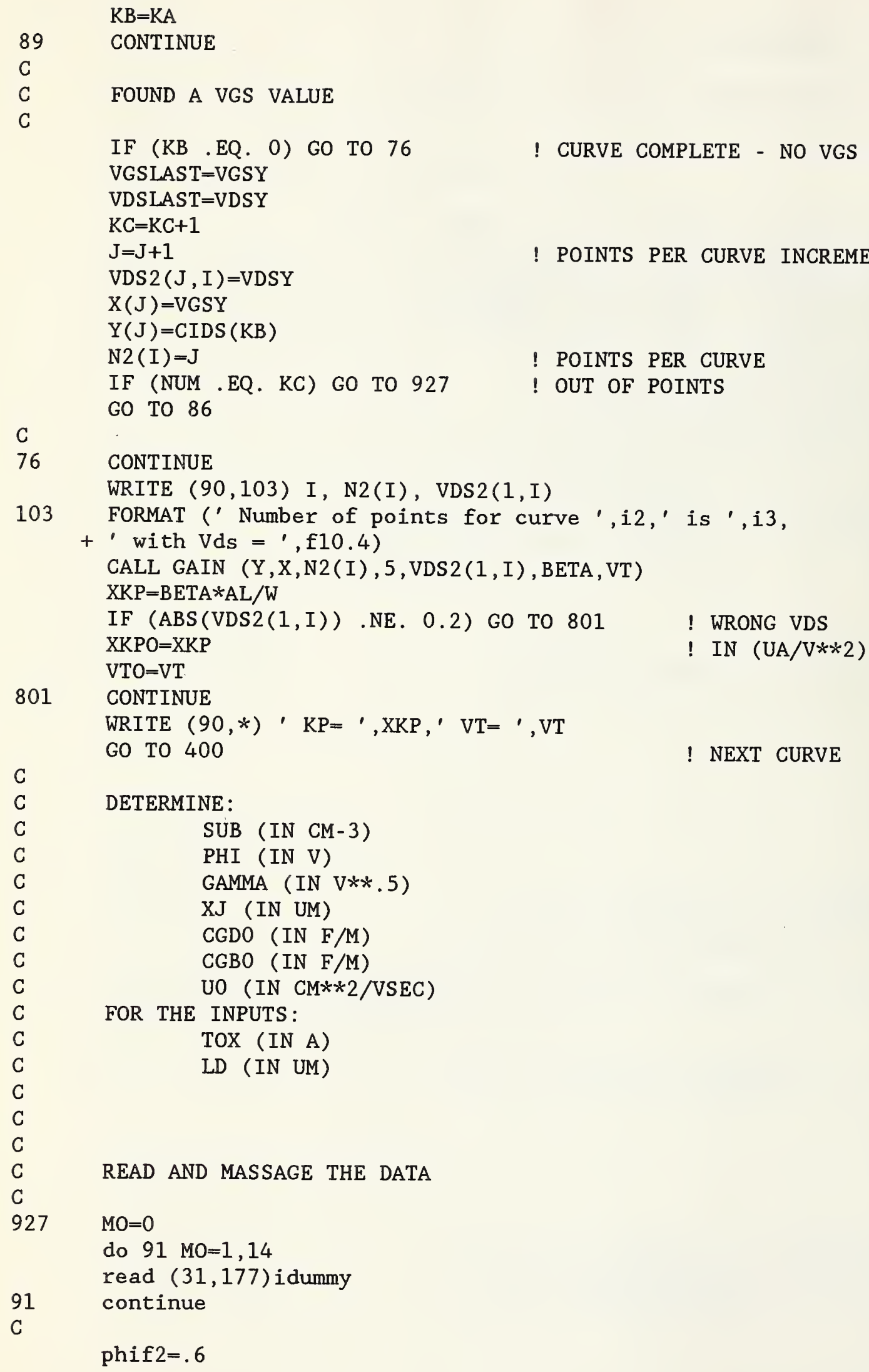


$\mathrm{KO}=0$

do $37 \mathrm{KO}=1,4$

read $(31,566$, end $=131)$ IDUM, IONE, $\operatorname{VBS}(\mathrm{KO})$

566 format (A4, I6, 1PE13.6)

read $(31,566$, end $=131$ ) IDUM, IONE, Y1(KO)

C* $\quad \operatorname{READ}(31, *, \mathrm{END}=131) \operatorname{VBS}(\mathrm{KO}), \mathrm{Y1}(\mathrm{KO})$

$\mathrm{X} 1(\mathrm{KO})=\operatorname{sqrt}(\operatorname{abs}(\mathrm{VBS}(\mathrm{KO}))+\mathrm{phif2})-\operatorname{sqrt}(\mathrm{phif2})$

$\mathrm{Y} 1(\mathrm{KO})=\mathrm{ABS}(\mathrm{Y} 1(\mathrm{KO}))$

write $(90, *) \operatorname{VBS}(\mathrm{KO}), \mathrm{X} 1(\mathrm{KO}), \mathrm{Y} 1(\mathrm{KO})$

C continue

cal1 $\operatorname{lnfit}(3,2, \mathrm{X} 1, \mathrm{Y} 1$, slopep, xint, rmspsub)

write $(90, *)$ 'slopep', slopep

$\mathrm{KO}=0$

do $47 \mathrm{KO}=5,8$

read $(31,566$, end $=131)$ IDUM, ITWO, $\operatorname{VBS}(\mathrm{KO})$

read $(31,566$, end $=131$ ) IDUM, ITWO, Y1(KO)

$\operatorname{READ}(31, *, \mathrm{END}=131) \mathrm{VBS}(\mathrm{KO}), \mathrm{Y} 1(\mathrm{KO})$

$C *$

$\mathrm{X} 1(\mathrm{KO})=\operatorname{sqrt}(\operatorname{abs}(\mathrm{VBS}(\mathrm{KO}))+\mathrm{phif2})-\operatorname{sqrt}(\mathrm{phif2})$

$\mathrm{Y} 1(\mathrm{KO})=\mathrm{ABS}(\mathrm{Y} 1(\mathrm{KO}))$

write $(90, *) \operatorname{VBS}(\mathrm{KO}), \mathrm{X} 1(\mathrm{KO}), \mathrm{Y} 1(\mathrm{KO})$

47 continue

cal1 lnfit (3,6,X1,Y1, slopen, xint, rmsnsub)

write $(90, *)$ 'slopen', slopen

C

C READ THE DATA

C

C

READ $(32,177)$ IDUMMY

\section{$\mathrm{IKEY}=32$}

$\mathrm{OKEY}=80$

130 READ (IKEY, *,$E N D=131$ ) tox, $x 1 d$

C

if (vdslast ge. 0.0) gamma=abs (slopen)

if (vdslast .1t. 0.0) gamma=abs (slopep)

if (vdslast.ge. 0.0) rmssub=rmsnsub

if (vdslast.1t. 0.0) rmssub=rmspsub

C write $(90, *)$ 'ikey=', ikey

C write $(90, *)$ 'gamma=', gamma

sub $=(1.0 e-6 /(2 . * e s * q)) *\left(\right.$ gamma*eox $\left./\left(\operatorname{tox}^{*} * 1.0 \mathrm{e}-10\right)\right) * * 2 \quad$ ! IN $(C M-3)$

C write $(90, *)$ 'sub $=$ ', sub

phi $=2.0 * .02585 * \operatorname{alog}($ sub/1.45e10)

$\mathrm{xj}=\mathrm{xld} / .8$

$c g d o=e o x * x 1 d * 1.0 e-6 /($ to $* * 1.0 e-10)$

! $\operatorname{IN}(F / M)$

cgso $=$ cgdo

cgbo $=$ eox*4.*1.0e-6/(2.*tox*1.0e-10)

$\mathrm{u} 0=(\mathrm{xkpo} *$ to $* 1.0 \mathrm{e}-8 * 1.0 \mathrm{e}-6) /\left(\mathrm{eox}^{*} 1.0 \mathrm{e}-2\right)$

! IN $((\mathrm{CM} * * 2) / \mathrm{V}-\mathrm{SEC})$

C

WRITE (OKEY, 375)

375 FORMAT (1X, 'LD')

WRITE (OKEY, 376) $\times 1 d$

376 FORMAT (1X, F5.3) 


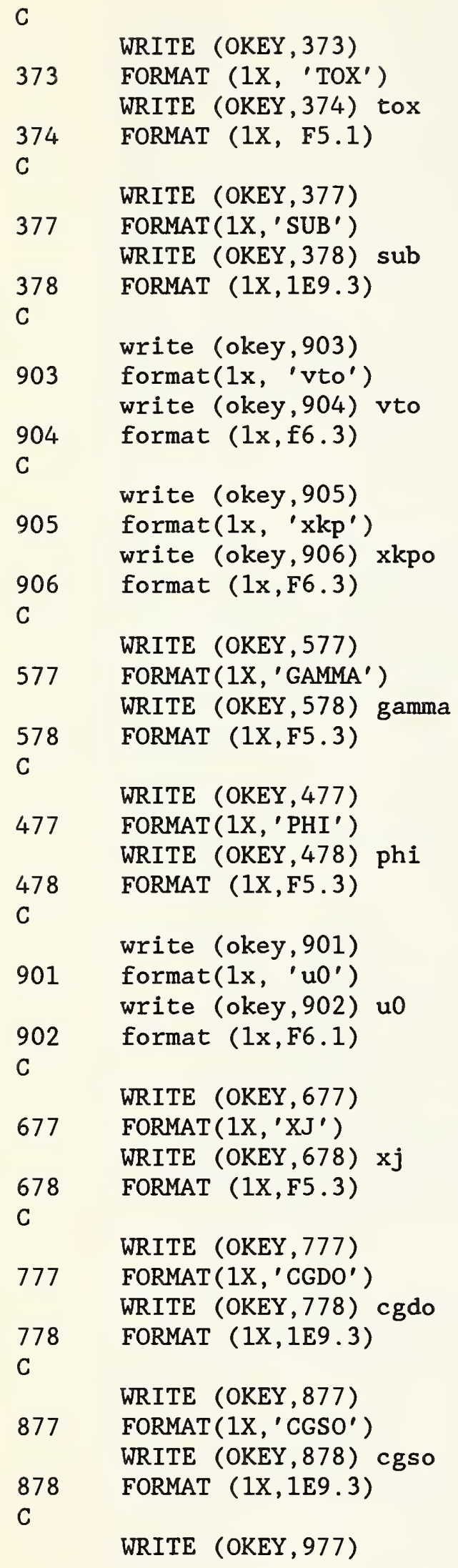




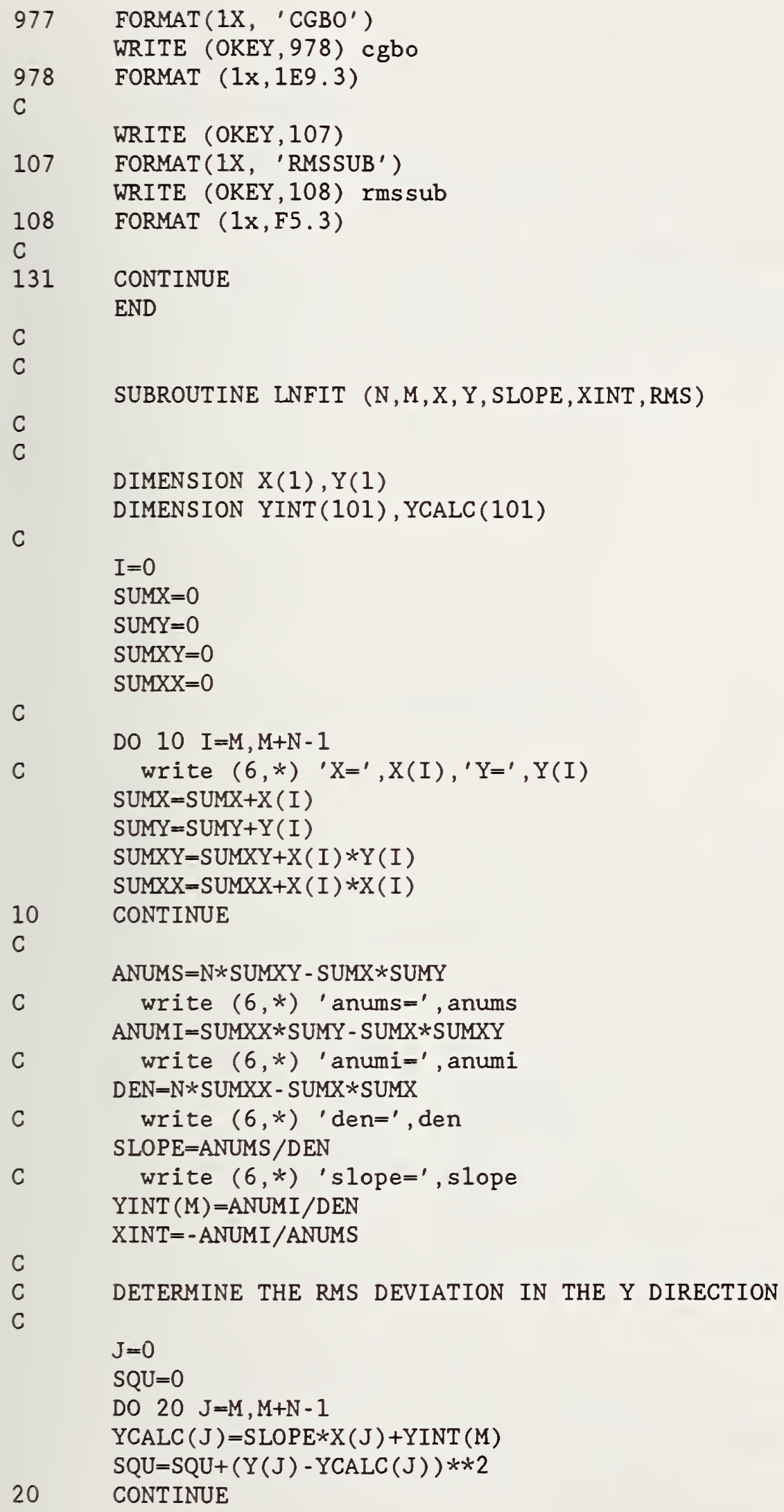

C

$I=0$

SUMX $=0$

SUMY $=0$

SUMXY $=0$

SUMXX $=0$

C

DO $10 I=M, M+N-1$

write $(6, *)^{\prime} \mathrm{X}={ }^{\prime}, \mathrm{X}(\mathrm{I}),{ }^{\prime} \mathrm{Y}={ }^{\prime}, \mathrm{Y}(\mathrm{I})$

C

SUMX $=S U M X+X(I)$

$S U M Y=S U M Y+Y(I)$

$\operatorname{SUMXY}=\operatorname{SUMXY}+X(I) * Y(I)$

$\operatorname{SUMXX}=\operatorname{SUMXX}+X(I) * X(I)$

10 CONTINUE

C

ANUMS $=\mathrm{N} *$ SUMXY - SUMX $*$ SUMY

$C$ write $(6, *)$ ' anums $=$ ' , anums

ANUMI $=S U M X X *$ SUMY - SUMX * SUMXY

C write $(6, *)$ 'anumi $=$ ', anumi

$D E N=N * S U M X X-S U M X * S U M X$

C write $(6, *)$ 'den=', den

SLOPE $=$ ANUMS $/$ DEN

write $(6, *)$ 'slope $=$ ', slope

C

$\operatorname{YINT}(\mathrm{M})=$ ANUMI $/$ DEN

XINT $=-$ ANUMI $/$ ANUMS

C

C DETERMINE THE RMS DEVIATION IN THE Y DIRECTION

C

$J=0$

$\mathrm{SQU}=0$

DO $20 \mathrm{~J}=\mathrm{M}, \mathrm{M}+\mathrm{N}-1$

$\operatorname{YCALC}(J)=S L O P E * X(J)+Y I N T(M)$

$S Q U=S Q U+(Y(J)-Y C A L C(J)) * * 2$

CONTINUE 


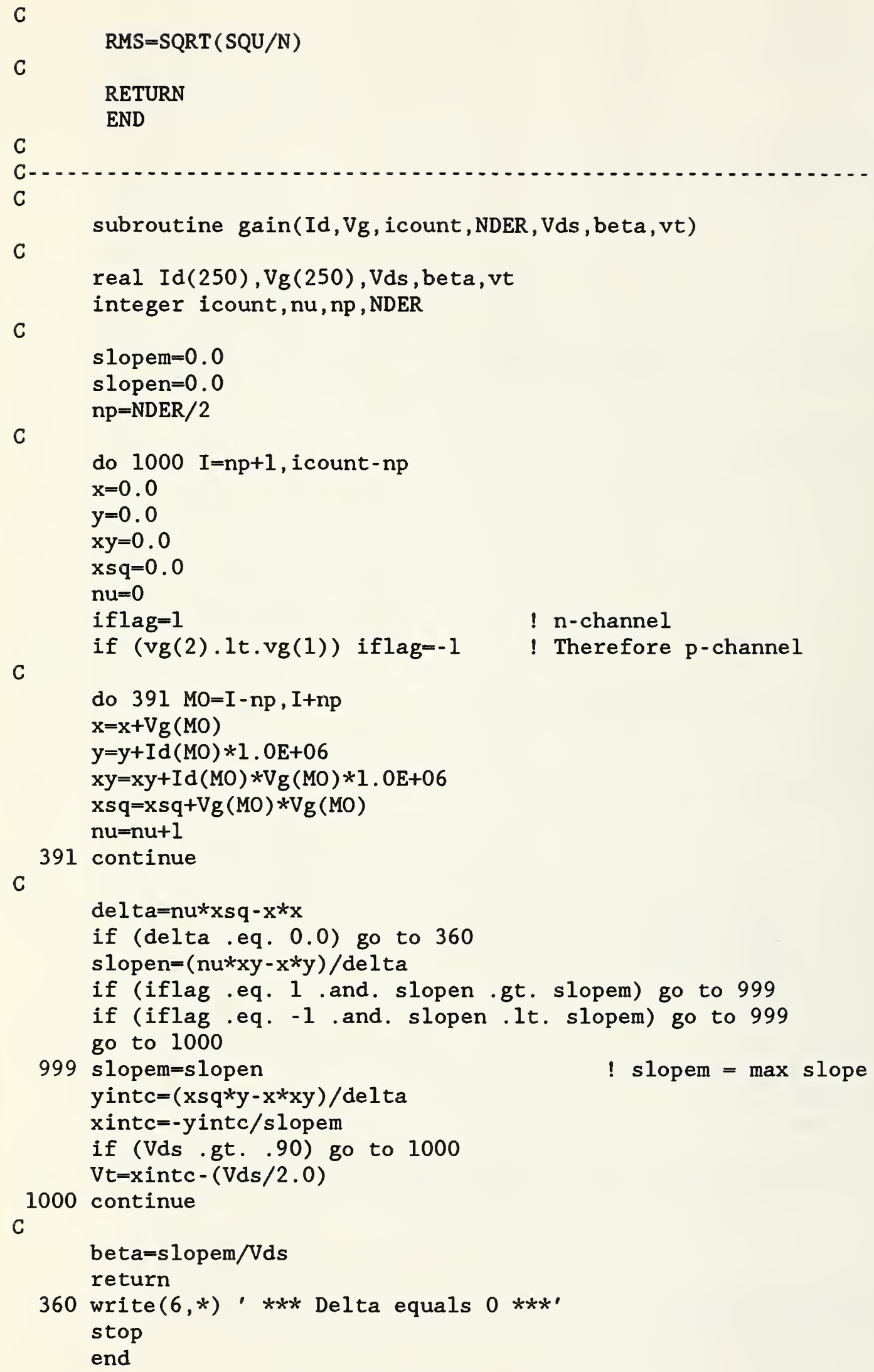


CAPKEY.FOR

C

C

FILENAME: CAPKEY.FOR

This program recalculates the capacitances given the optimized parameters from SUXES.

open (unit $=20, f i l e={ }^{\prime}$ guest. dat', status='old' )

open (unit=40, file='expar.dat', status='old')

open(unit=60, file='parspice.dat', status='unknown')

C
$\mathrm{EOX}=34.5 \mathrm{E}-12$
! in $\mathrm{F} / \mathrm{m}$

C

C

C

READ THE PRESUXES DATA

$$
\begin{aligned}
& \mathrm{KEY}=20 \\
& \mathrm{k}=0
\end{aligned}
$$

do $100 \mathrm{k}=1,25$

read (key, 377 , end $=331$ ) dummy

377 format (A1)

100 continue

C

READ (KEY,*,END=331) DRMSSUB

C

READ THE AFTSUXES DATA

C

331 IKEY $=40$

READ (IKEY, * END=231) XLD, trash1, trash2

READ (IKEY, *,END=231) TOX, trash1, trash2

READ (IKEY, *, END=231) XNSUB, trash1, trash2

READ (IKEY, *,END=231) VTO, trash1, trash2

READ (IKEY, *,END=231) XKP, trash1, trash2

$\mathrm{XKP}=\mathrm{XKP} * 1.0 \mathrm{E}+6$

READ (IKEY, *, END=231) GAMMA, trash1, trash2

READ (IKEY, *,END=231) PHI, trash1, trash2

READ (IKEY, *, END=231) UO, trash1, trash2

READ (IKEY, *, END=231) DELTA, trash1, trash2

READ (IKEY, *, END=231) VMAX, trash1, trash2

READ (IKEY, *, END=231) XJ , trash1, trash2

READ (IKEY, *,END=231) XKAPPA, trash1, trash2

READ (IKEY, *, END=231) XNFS, trash1, trash2

READ (IKEY, *,END=231) ETA, trash1, trash2

READ (IKEY, *, END=231) THETA, trash1, trash2

C

RECALCULATE THE CAPACITANCES

cgdo $=e o x * x 1 d * 1.0 e-6 /(\operatorname{tox} * 1.0 e-10)$

cgso $=$ cgdo

cgbo $=$ eox $* 4 . * 1.0 \mathrm{e}-6 /(2 . * \operatorname{tox} * 1.0 \mathrm{e}-10)$

ATOX $=$ TOX $* 1 e-4$ 


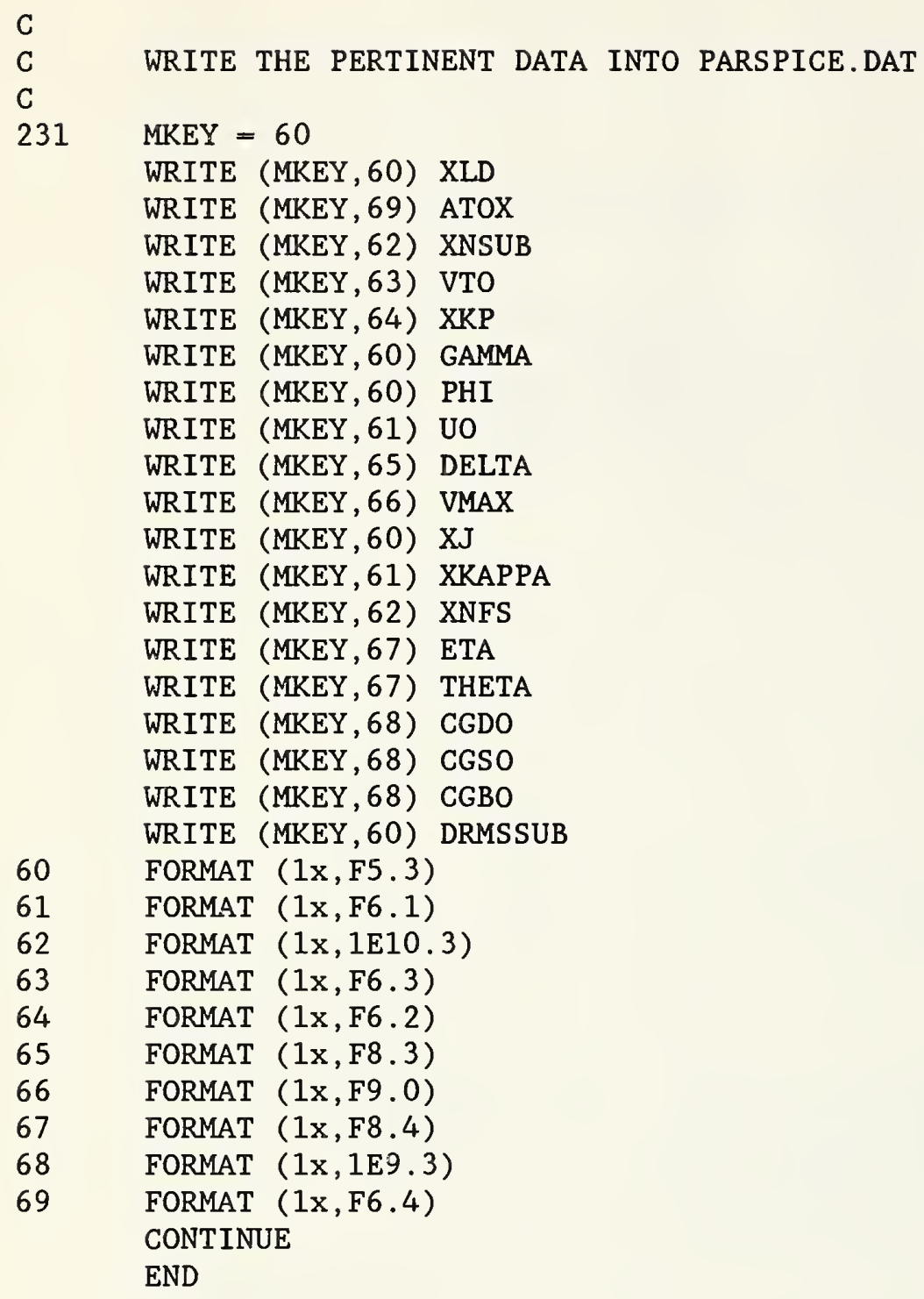




\section{IVKEY, FOR}

C

C

C

C

C

C

C

C

C

C

600

JKEY $=0$

DIMENSION VDS (1000), VGS(1000), $\operatorname{VBS}(1000)$

DIMENSION CIDS (1000), CNVBS (10)

DIMENSION VD(1000), CID(1000)

open (unit $=10$, file='in.dat', status='old')

open(unit=20, file $=^{\prime}$ in2.dat', status $={ }^{\prime}$ old' )

open (unit $=30$, file='in3.dat', status='old')

open (unit $=40$, file='in4.dat', status='old')

open (unit $=11$, file='out.dat', status='unknown')

open (unit $=21$, file='out2.dat', status=' unknown')

open (unit=31, file='out 3 .dat', status=' unknown')

open (unit=41, file='out4.dat', status=' unknown')

open (unit $=42$, file $=$ ' out 5 .dat', status=' unknown' )

open (unit=43, file='out6.dat', status=' unknown')

open (unit $=44$, file $=^{\prime}$ out 7 .dat', status=' unknown')

open (unit $=45$, file $=$ ' out 8 .dat', status=' unknown' )

open (unit $=46$, file='out9.dat', status='unknown' )

READ THE DATA

$\mathrm{JKEY}=\mathrm{JKEY}+10$

$\mathrm{J}=0$

DO $378 \mathrm{~J}=1,3$

READ (JKEY, 377) IDUMMY

377 FORMAT (A1)

378 CONTINUE

IF (JKEY .LT. 30) GO TO 600

READ $(40,377)$ IDUMMY

$\operatorname{READ}(40, *) \mathrm{N}$

$\mathrm{N}=\mathrm{N} / 6$

C

C

C

500

IKEY $=0$

MASSAGE THE DATA FILES AND THE FILES FROM SUXES

$\mathrm{L}=0$

IKEY $=I K E Y+10$

$I=0$

$230 \quad \mathrm{I}=\mathrm{I}+1$

$\operatorname{READ}(\operatorname{IKEY}, *, \operatorname{END}=231) \operatorname{VDS}(I), \operatorname{VGS}(I), \operatorname{VBS}(I), \operatorname{CIDS}(I)$

$\mathrm{AN}=0.0$

$100 \quad \mathrm{AN}=\mathrm{AN}+1.0$

IF (AN . GT. 5.5) GO TO 230

IF $(A B S(\operatorname{VGS}(I))$.LT. $(($ AN -1.0$)+.99))$ GO TO 230 


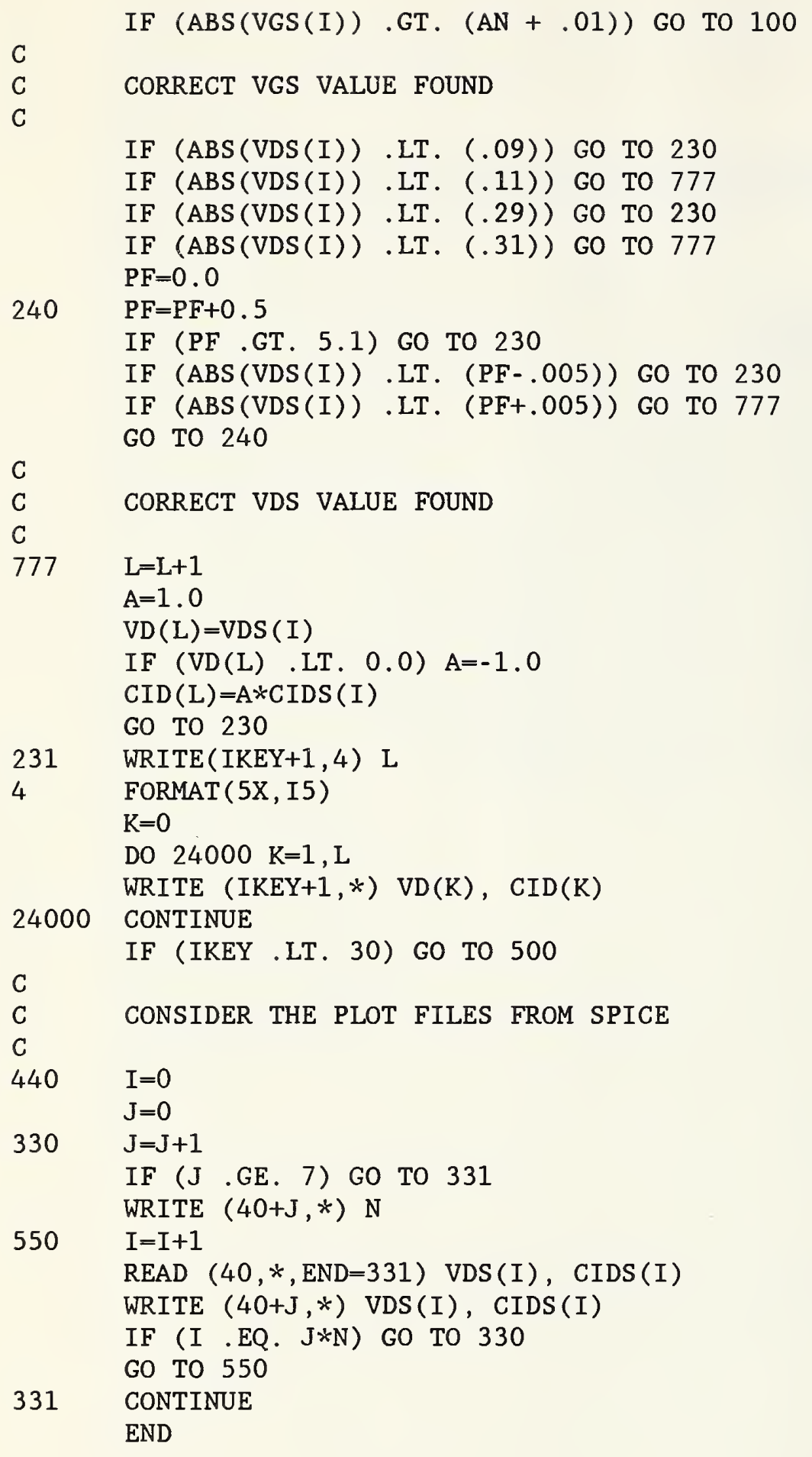


SUMKEY. FOR

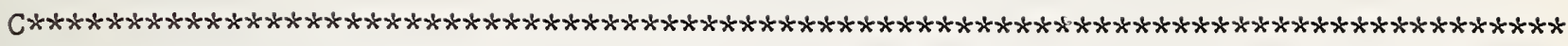

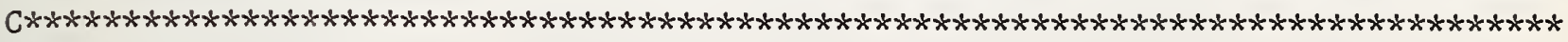
C

C

C

C

C

FILENAME: SUMKEY.FOR PURPOSE: THIS PROGRAM ADDS TO THE 'RESULTS.DAT' FILE AND CALCULATES
THE AVERAGES.

dimension chip(50), wave(50), wsum(50)

dimension alave(50), alsum(50)

C

common /plomi/ MARK, IWAF(100), lotnum1, lotnum2

C

common map $(10,10)$

character*1 IWAF

character*1 IW

character* 1 IWAFCUR

character* 1 MARK

character*4 lotnum 1

character*4 lotnum2

C

open (unit=10, file='mapl.DAT', status='unknown' )

open (unit $=20$, name $=$ 'in.dat', status $={ }^{\prime}$ old' )

open (unit $=24$, file='nlw.fil', status='old')

open (unit=25, file='line.fil', status='old')

open (unit=30, name $=$ ' out.dat', status='unknown' )

open (unit=49, file='wp.dat', status='unknown')

open (unit $=50$, file='lp.dat', status='unknown')

open (unit=51, file='w. dat', status=' unknown' )

open (unit $=52$, file='1. dat', status='unknown')

open (unit=53, file='mckt.dat', status='unknown' )

open (unit=54, file='sckt.dat', status='unknown')

open (unit=55, file='pdiff.dat', status='unknown')

open (unit=56, file='nvto.dat', status='unknown' )

open (unit=57, file='nkp.dat', status='unknown' )

open (unit=58, file='ntox.dat', status='unknown' )

open(unit=59, file='nld.dat', status='unknown' )

open (unit $=60$, file='nsub.dat', status='unknown' )

open (unit=61, file='nrms.dat', status='unknown' )

open (unit=62, file='nphi.dat', status='unknown' )

open (unit=63, file='ngamma.dat', status='unknown' )

open (unit=64, file='nxj.dat', status='unknown')

open (unit=65, file='ncgdo.dat', status='unknown' )

open (unit=66, file='ncgso.dat', status='unknown')

open (unit=67, file='ncgbo.dat', status='unknown')

open (unit=68, file='nerr.dat', status='unknown')

open (unit=69, file='ntheta.dat', status='unknown')

open (unit $=70$, file='nkappa.dat', status='unknown' )

open (unit=71, file='ndelta.dat', status='unknown') 


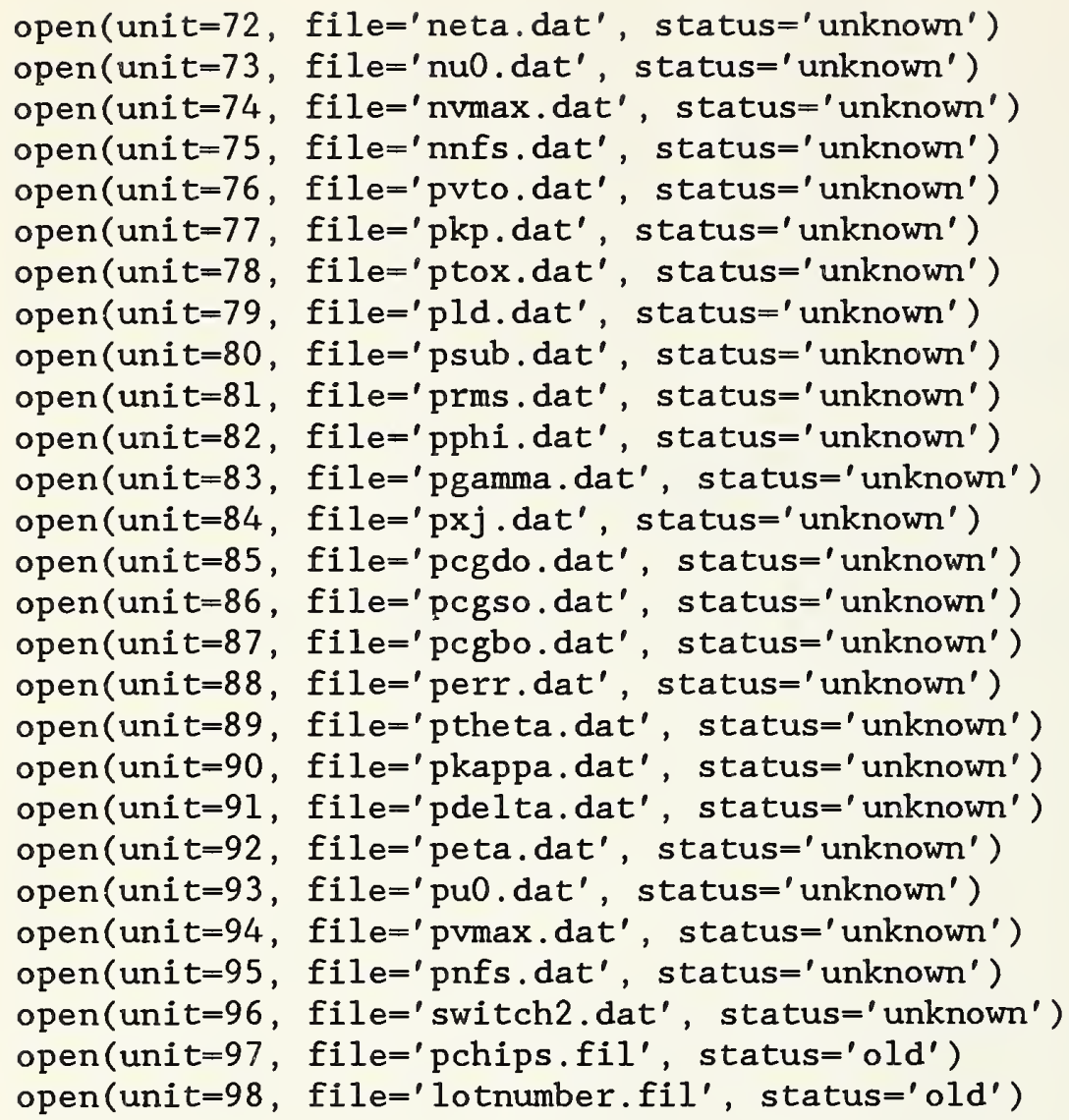




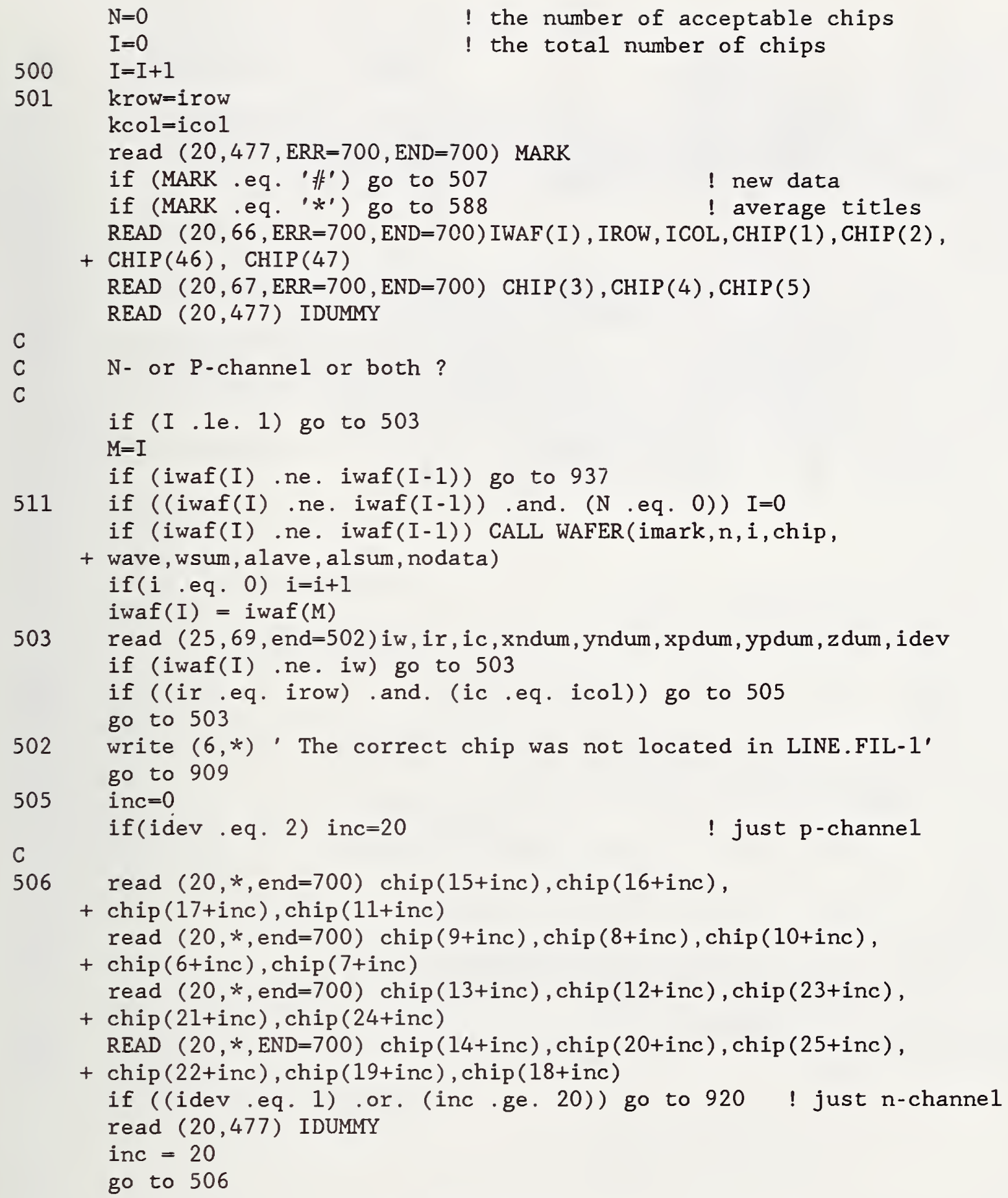


C

$920 \quad z$ fil $=0.0$

if (mark .eq. ' $-{ }^{\prime}$ ) imark $=-1$

if (mark .eq. ' + ') imark=1

write $(30,477)$ MARK

WRITE $(30,41)$ IWAF(I), IROW, ICOL, chip (1), chip (2), chip (46), chip (47)

WRITE $(30,67)$ chip(3), chip (4), chip(5)

$\operatorname{WRITE}(30, *)$ ' '

inc $=0$

if (idev .eq. 2) inc $=20$

538 write $(30,42) \operatorname{chip}(15+i n c), \operatorname{chip}(16+i n c), \operatorname{chip}(17+i n c), \operatorname{chip}(11+i n c)$

write $(30,43)$ chip $(9+i n c), \operatorname{chip}(8+i n c), \operatorname{chip}(10+i n c)$,

$+\operatorname{chip}(6+i n c), \operatorname{chip}(7+i n c)$

WRITE $(30,44)$ chip (13+inc), chip (12+inc), chip (23+inc),

$+\operatorname{chip}(21+i n c), \operatorname{chip}(24+i n c)$

WRITE $(30,45) \operatorname{chip}(14+i n c), \operatorname{chip}(20+i n c), \operatorname{chip}(25+i n c)$,

$+\operatorname{chip}(22+i n c), \operatorname{chip}(19+i n c), \operatorname{chip}(18+i n c)$

if ((idev .eq. 1) .or. (inc.ge. 20)) go to 283

write $(30,47)$

inc $=20$

go to 538

477 format (1x, A1)

$C$ For the row and column - row 非

41 format ( $5 \mathrm{x}, \mathrm{A1}, 1 \mathrm{x}, \mathrm{I} 3, \mathrm{I} 4,1 \mathrm{x}, \mathrm{F} 5.2,6 \mathrm{x}, \mathrm{F} 5.2,6 \mathrm{x}, \mathrm{F} 5.2,6 \mathrm{x}, \mathrm{F} 5.2$ )

C For row 非 2

42 format $(15 \mathrm{x}, 1 \mathrm{e} 10.3,1 \mathrm{x}, 1 \mathrm{e} 10.3,1 \mathrm{x}, 1 \mathrm{e} 10.3,2 \mathrm{x}, \mathrm{F} 5.3)$

C For row 非 3

43 format (16x, F5.3, 3x, F6.1, 7x, 1e10.3, 1x, F6. 3, 2x, F6. 2)

C For the SUXES parameters - row 非 4

44 FORMAT (16X, F5 . 3, 6x, F5 . 3, 3x, F6.1, 5x, F8 . 3, 3x, 1e10.3)

C For the last row

45 format ( $16 \mathrm{x}, \mathrm{F} 5.3,3 \mathrm{x}, \mathrm{F} 6.1,7 \mathrm{x}, 1 \mathrm{e} 10.3, \mathrm{~F} 8.4, \mathrm{~F} 8.3,4 \mathrm{x}, \mathrm{F} 6.2$ )

C Similar to 41

46 format ( $5 x, A 1,2 x, F 5.2,2 x, F 5.2,6 x, F 5.2,6 x, F 5.2,5 x, F 6.2)$

47 format $(1 \mathrm{x}$, ' ')

49 format ( $8 \mathrm{x}, \mathrm{F5} .2,2 \mathrm{x}, \mathrm{F} 5.2,6 \mathrm{x}, \mathrm{F} 5.2,6 \mathrm{x}, \mathrm{F} 5.2,5 \mathrm{x}, \mathrm{F} 6.2$ )

66 format $(5 \mathrm{x}, \mathrm{A} 1,1 \mathrm{x}, \mathrm{I} 3, \mathrm{I} 4,1 \mathrm{x}, \mathrm{F} 5.2,6 \mathrm{x}, \mathrm{F} 5.2,6 \mathrm{x}, \mathrm{F} 5.2,6 \mathrm{x}, \mathrm{F} 5.2)$

67 format $(15 \mathrm{x}, \mathrm{F} 5.2,6 \mathrm{x}, \mathrm{F} 5.2,5 \mathrm{x}, \mathrm{F} 6.2)$

69 format $(2 \mathrm{x}, \mathrm{A} 1,2 \mathrm{x}, \mathrm{I} 2,1 \mathrm{x}, \mathrm{I} 2,1 \mathrm{x}, \mathrm{F} 5.2,1 \mathrm{x}, \mathrm{F} 5.2,1 \mathrm{x}, \mathrm{F} 5.2,1 \mathrm{x}$,

$+\mathrm{F} 5.2,1 \mathrm{x}, \mathrm{F} 5.2,1 \mathrm{x}, \mathrm{I} 3)$

ignore the averages from last time
$588 \quad \begin{aligned} & \text { IO }=0 \\ & \text { do } 578 \text { I0 }=1,24 \\ & \text { read }(20,477) \text { IDUMMY } \\ & \text { continue } \\ & \text { go to } 501\end{aligned}$
$\begin{aligned} & \text { Codata }=1 \\ & \text { Go T0 } 707\end{aligned}$




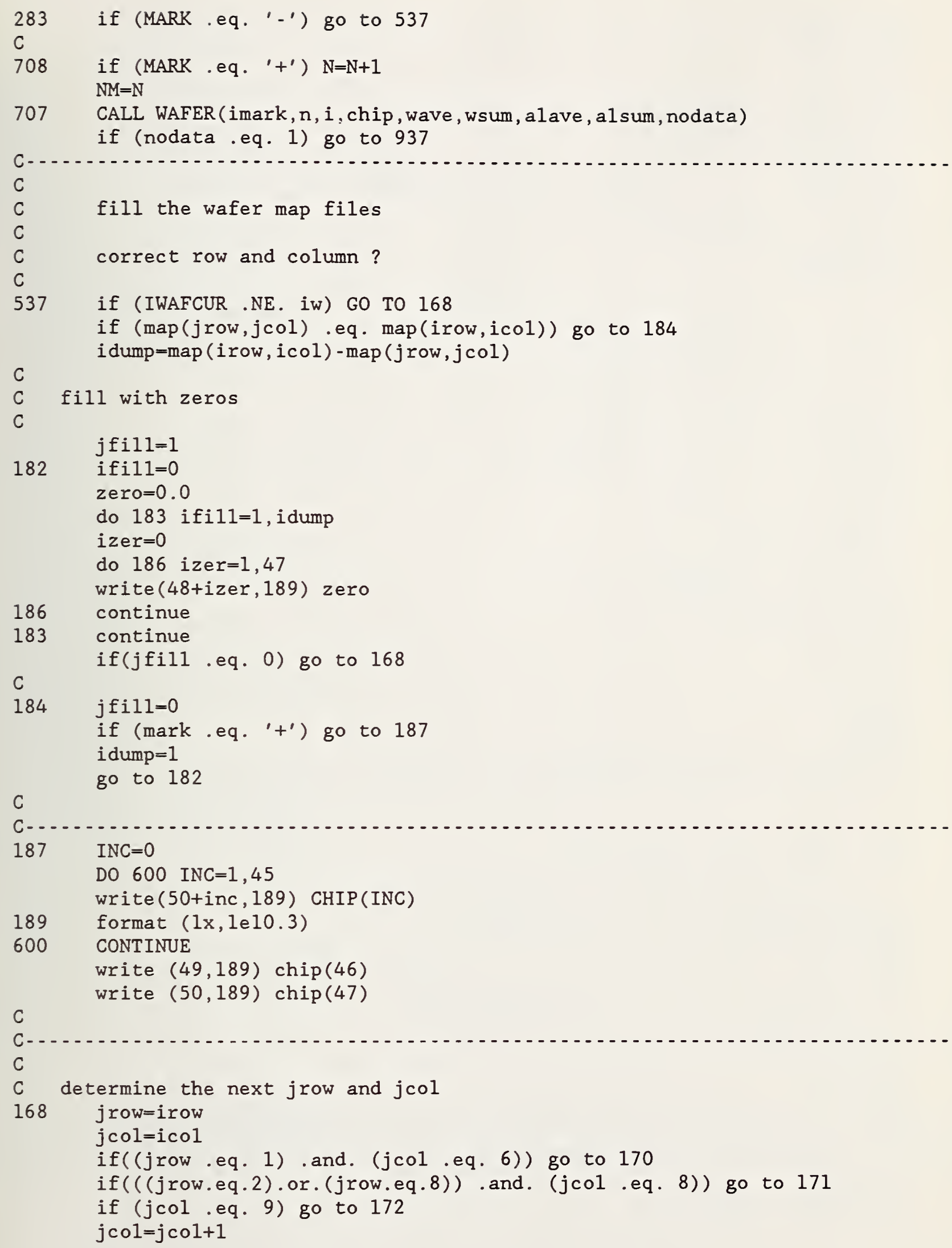




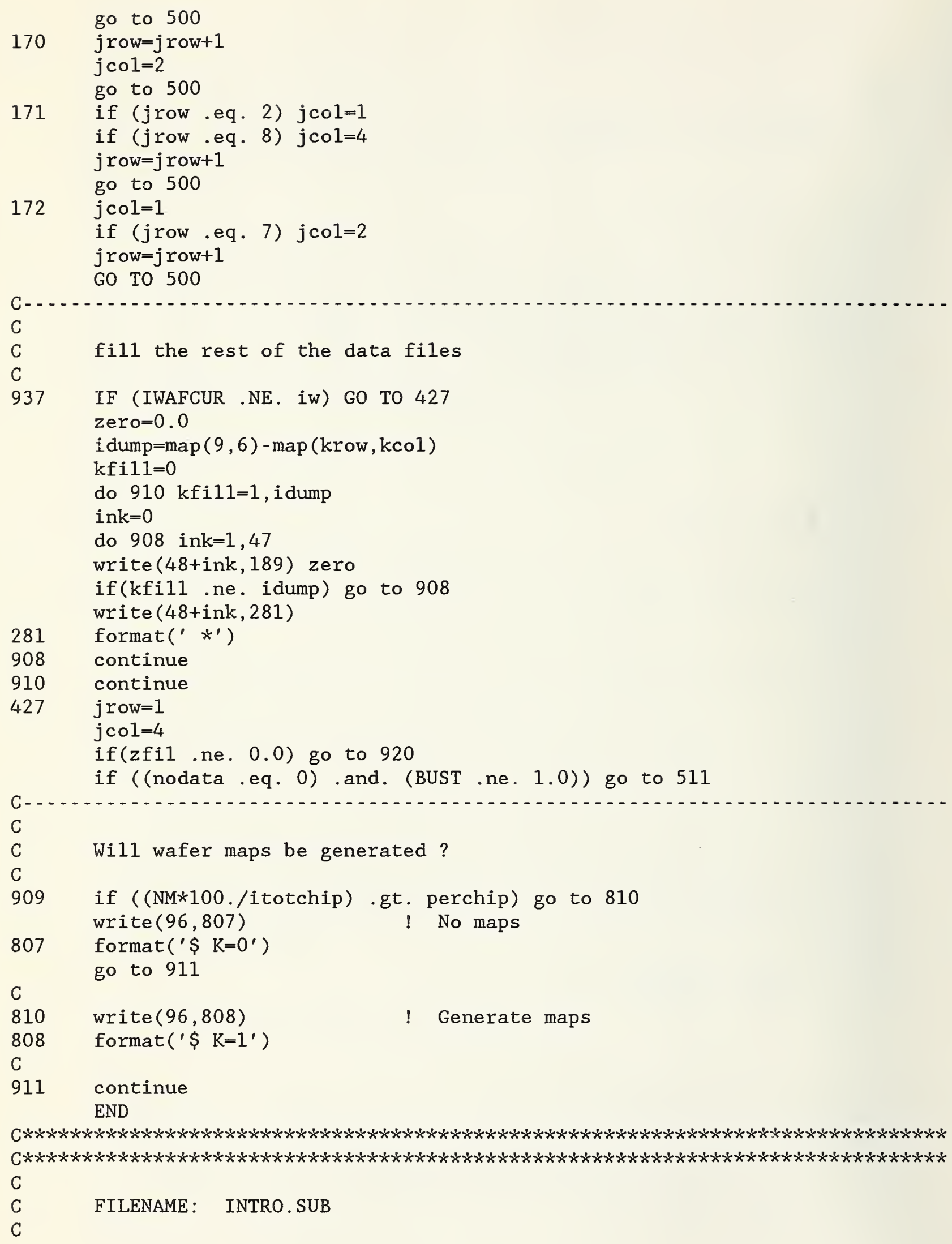


C

SUBROUTINE INTRO (chip, wave, wsum, alave, alsum, perchip,

+ itotchip)

C

dimension chip(1), wave(1), wsum(1)

dimension alave(1), alsum(1)

C

common /plomi/ MARK, $\operatorname{IWAF}(100)$, lotnum1, lotnum2

common map $(10,10)$

C

character*1 IWAF

character*1 IW

character $* 1$ MARK

character*4 lotnuml

character*4 lotnum2

C

C

C

C

FILL THE MAP DIMENSION TO NUMBER THE CHIPS

jmap $=0$

do 160 jmap $=4,6$

$\operatorname{map}(1, j$ map $)=$ jmap -3

$\operatorname{map}(9, j$ map $)=j$ map +59

160 continue

$j$ map $=0$

do 161 jmap $=2,8$

$\operatorname{map}(2, j$ map $)=j$ map +2

$\operatorname{map}(8, j$ map $)=j$ map +54

161 continue

jmap $=0$

do 162 jmap $=1,9$

$\operatorname{map}(3, j$ map $)=j$ map +10

$\operatorname{map}(4, j$ map $)=j$ map +19

$\operatorname{map}(5, j$ map $)=$ jmap +28

$\operatorname{map}(6, j$ map $)=j$ map +37

$\operatorname{map}(7$, jmap $)=$ jmap +46

162 continue

read $(97,477)$ IDUMMY

read $(97, *)$ perchip, itotchip

C

C READ THE DUMMY DATA

C

$\mathrm{MO}=0$

DO $378 \mathrm{MO}=1,13$

$\operatorname{READ}(20,477)$ IDUMMY

477 format ( $1 x, A 1)$

378 CONTINUE

C

$$
\begin{aligned}
& \mathrm{mo}=0 \\
& \text { do } 478 \mathrm{mo}=1,5
\end{aligned}
$$




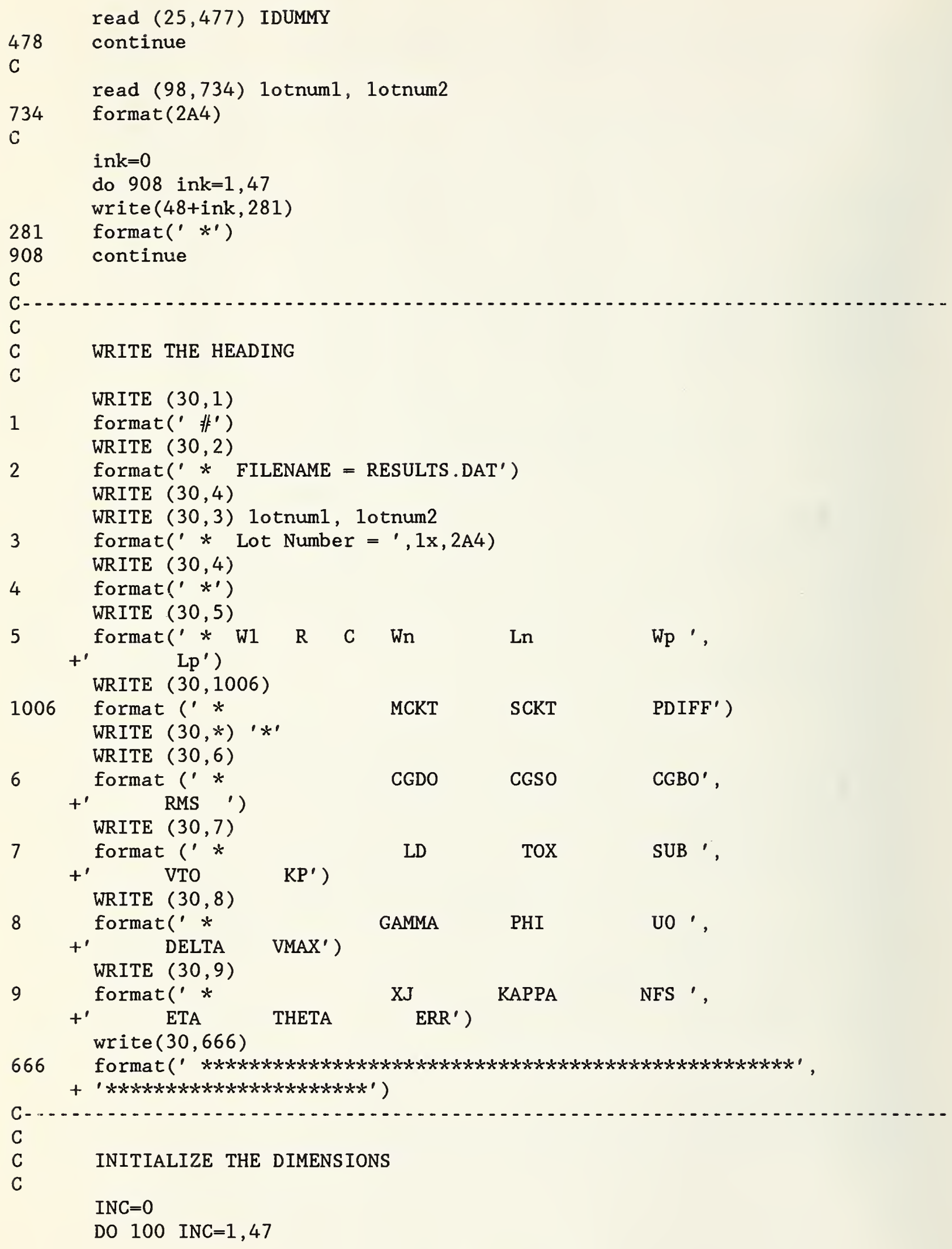




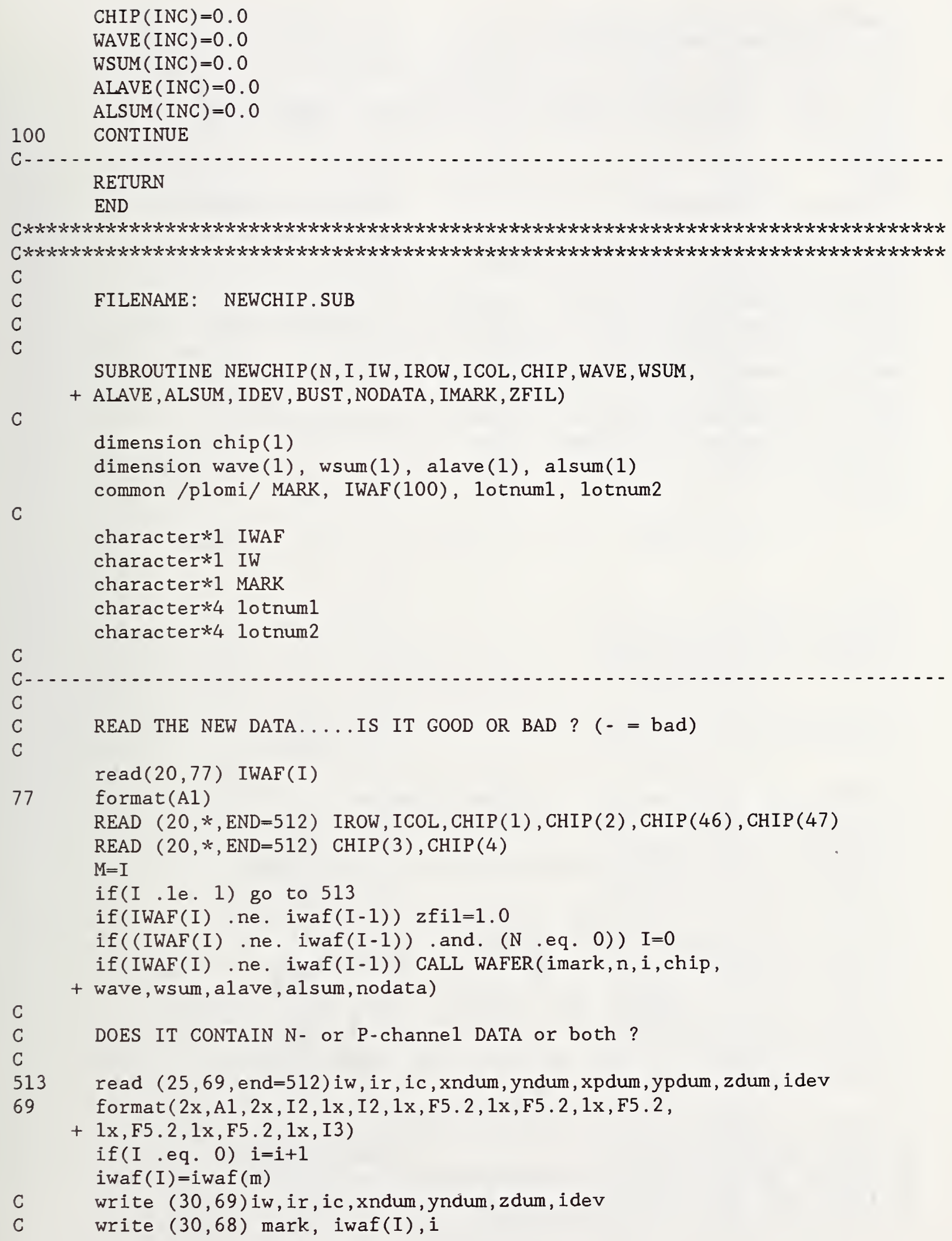


C68 format ( $1 \mathrm{x}, \mathrm{A1}, 1 \mathrm{x}, \mathrm{A1}, 1 \mathrm{x}, \mathrm{I2})$

if (iwaf(I) .ne. iw) go to 513

if ((ir .eq. irow) .and. (ic .eq. icol)) go to 515

go to 513

512 write $(6, *)$ ' The correct chip was not located in LINE.FIL-2' BUST $=1.0$

RETURN

515 inc $=0$

if (idev .eq. 2) inc=20 ! just p-channe1

c

$516 \operatorname{read}(20, *$, end $=512) \operatorname{chip}(9+i n c), \operatorname{chip}(8+i n c), \operatorname{chip}(10+i n c)$,

$+\operatorname{chip}(6+i n c), \operatorname{chip}(7+i n c)$

chip $(8+i n c)=\operatorname{chip}(8+i n c) * 1 . e 4$

read $(20, *$, end $=512) \operatorname{chip}(13+i n c), \operatorname{chip}(12+i n c), \operatorname{chip}(23+i n c)$,

$+\operatorname{chip}(21+i n c), \operatorname{chip}(24+i n c)$

read $(20, *$, end $=512) \operatorname{chip}(14+i n c), \operatorname{chip}(20+i n c), \operatorname{chip}(25+i n c)$,

$+\operatorname{chip}(22+i n c), \operatorname{chip}(19+i n c)$

READ $(20, *, E N D=512) \operatorname{chip}(15+i n c), \operatorname{chip}(16+i n c), \operatorname{chip}(17+i n c)$,

$+\operatorname{chip}(11+i n c)$, trash, chip (18+inc), trash

if ((idev .eq. 1) .or. (inc .ge. 20)) go to 517 ! just n-channel

inc $=20$

$\mathrm{C}$ go to 516

\section{C \\ C}

$517 \quad$ IFLG $=0$

IF ( $\operatorname{chip}(3)$.EQ 0.0 ) IFLG=1

! $3=\mathrm{MCKT}$

if (IFLG .GE. 1) go to 607

C

$\operatorname{chip}(5)=100 *(\operatorname{ABS}(\operatorname{chip}(3)-\operatorname{chip}(4))) / \operatorname{chip}(3) \quad ! 4=\operatorname{SCKT}$

if $((\operatorname{chip}(5)$.LT, 0.0$)$.OR. $(\operatorname{chip}(5)$.GT, 100.0$))$ IFLG=2

C

if (IFLG.GE. 1) go to $607 \quad$ ! $5=$ PDIFF

if (idev .eq. 2) go to 556 ! just p-channel $6=$ NVTO

if $((\operatorname{chip}(6)$.LE. 0.3) .OR. $(\operatorname{chip}(6)$.GE. 1.5)) IFLG=3

if (IFLG.GE. 1) go to $607 \quad ! 7=$ NKP

if $((\operatorname{chip}(7)$.LE. 1.0) .OR. ( $\operatorname{chip}(7)$.GE. 100.0)) IFLG=4

if (IFLG .GE. 1) go to $607 \quad$ ! $8=$ NTOX

if $((\operatorname{chip}(8)$.LE. 250.0) .OR. ( $\operatorname{chip}(8)$.GE. 1000.0)) IFLG=5

if (IFLG.GE. 1) go to $607 \quad$ ! $9=$ NLD

if $((\operatorname{chip}(9)$.LE. 0.0) .OR. ( $\operatorname{chip}(9)$.GE. 5.0) ) IFLG=6

if (IFLG .GE. 1) go to $607 \quad ! 10=$ NSUB

if $((\operatorname{chip}(10)$.LE. 1.0E13) .OR. (chip(10) .GE. 1.0E17)) IFLG=7

if (IFLG .GE. 1) go to 607 ! $11=$ NRMS

if $((\operatorname{chip}(11) . \mathrm{LT}, 0.0)$.OR. ( $\operatorname{chip}(11)$.GT 0.20$))$ IFLG=7

C

if (IFLG.GE. 1) go to $607 \quad$ ! $12=$ NPHI

if $((\operatorname{chip}(12)$.LE. 0.3$)$.OR. ( $\operatorname{chip}(12)$, GE .0 .9$))$ IFLG=8

if (IFLG.GE. 1) go to $607 \quad$ ! $13=$ NGAMMA

if $((\operatorname{chip}(13)$.LE. 0.1) .OR. ( $\operatorname{chip}(13)$.GE .3 .0$))$ IFLG=9

if (IFLG .GE. 1) go to $607 \quad ! 14=$ NXJ

if $((\operatorname{chip}(14) . \mathrm{LE} \cdot 0.0)$.OR. (chip(14) .GE. 1.5)) IFLG=10 
if (IFLG.GE. 1) go to 607

! $15=$ NCGDO

if $((\operatorname{chip}(15) . \mathrm{LE}, 0.0) . \mathrm{OR} .(\operatorname{chip}(15) . \mathrm{GE}, 1.0 \mathrm{E}-7))$ IFLG=11

if (IFLG.GE. 1) go to 607

! $16=$ NCGSO

if ( $(\operatorname{chip}(16)$.LE. 0.0) .OR

1. OE - 7)) IFLG $=12$

if (IFLG .GE. 1) go to 607

! $17=$ NCGBO

if $((\operatorname{chip}(17)$.LE. 0.0) .OR. ( $\operatorname{chip}(17)$.GE. 1.0E-7)) IFLG=13

if (IFLG .GE. 1) go to 607

! $18=$ NERR

if $((\operatorname{chip}(18)$.LT .0 .0$)$.OR. $(\operatorname{chip}(18)$.GT .10 .0$))$ IFLG=13

C

if (IFLG.GE. 1) go to 607

! $19=$ NTHETA

if $((\operatorname{chip}(19) . L E .-2.0)$. OR. ( $\operatorname{chip}(19) . \mathrm{GE} .2 .0))$ IFLG=14

if (IFLG .GE. 1) go to 607 ! $20=$ NKAPPA

if $((\operatorname{chip}(20)$.LE. 0.0) .OR. ( $\operatorname{chip}(20)$. .GE. 999.0)) IFLG=15

if (IFLG.GE. 1) go to 607 ! $21=$ NDELTA

if $((\operatorname{chip}(21)$.LE. -100.0$)$.OR. ( $\operatorname{chip}(21) . \mathrm{GE} .100 .0))$ IFLG=16

if (IFLG .GE. 1) go to 607 ! $22=$ NETA

if $((\operatorname{chip}(22)$.LE. -2.0$)$.OR. ( $\operatorname{chip}(22) . \mathrm{GE} .2 .0))$ IFLG=17

if (IFLG .GE. 1) go to $607 \quad ! 23=$ NUO

if $((\operatorname{chip}(23)$.LE. 0.1) .OR. ( $\operatorname{chip}(23)$.GE. 1250.0)) IFLG=18

if (IFLG .GE. 1) go to 607 ! $24=$ NVMAX

if $((\operatorname{chip}(24)$.LE. 1.0e4) .OR. ( $\operatorname{chip}(24) . \mathrm{GE} .9 .9 \mathrm{e} 7))$ IFLG=19

if (IFLG .GE. 1) go to $607 \quad$ ! $25=$ NNFS

if $((\operatorname{chip}(25)$.LE. 0.0) .OR. ( $\operatorname{chip}(25) . \mathrm{GE} .4 .0 \mathrm{e} 14))$ IFLG=20

C

if (IFLG .GE. 1) go to 607

! $26=$ PVTO

if (idev .eq. 1) go to 557 ! just n-channel

556 if $((\operatorname{chip}(26)$.LE. -3.0$)$.OR. ( $\operatorname{chip}(26)$.GE. -0.1$))$ IFLG=21

if (IFLG.GE. 1) go to $607 \quad$ ! $27=$ PKP

if $((\operatorname{chip}(27)$.LE. 1.0) .OR. $(\operatorname{chip}(27)$.GE. 100.0)) IFLG=22

if (IFLG .GE. 1) go to $607 \quad ! 28=$ PTOX

if ((chip (28) .LE. 250.0) .OR. (chip(28) .GE. 1000.0)) IFLG=23

if (IFLG .GE. 1) go to 607 ! $29=$ PLD

if $((\operatorname{chip}(29)$.LE. 0.0) .OR. $(\operatorname{chip}(29)$.GE. 5.0)) IFLG=24

if (IFLG.GE. 1) go to 607 ! $30=$ PSUB

if $((\operatorname{chip}(30)$.LE. 1.0E13) .OR. ( $\operatorname{chip}(30) . \mathrm{GE} .1 .0 \mathrm{E} 17))$ IFLG=25

if (IFLG.GE. 1) go to $607 \quad$ ! $31=$ PRMS

if $((\operatorname{chip}(31)$.LT. 0.0) .OR. (chip(31) .GT. 0.20)) IFLG=25

if (IFLG.GE. 1) go to $607 \quad$ ! $32=$ PPHI

C

if $((\operatorname{chip}(32)$.LE. 0.3$)$.OR. ( $\operatorname{chip}(32) . \mathrm{GE} .0 .9))$ IFLG=26

if (IFLG.GE. 1) go to 607 ! $33=$ PGAMMA

if $((\operatorname{chip}(33)$.LE. 0.1$)$.OR. $(\operatorname{chip}(33)$.GE .3 .0$))$ IFLG=27

if (IFLG.GE. 1) go to $607 \quad$ ! $34=$ PXJ

if $((\operatorname{chip}(34)$.LE. 0.0) .OR. ( $\operatorname{chip}(34)$.GE. 1.5)) IFLG=28

if (IFLG.GE. 1) go to 607 ! $35=$ PCGDO

if $((\operatorname{chip}(35)$.LE. 0.0) .OR. ( $\operatorname{chip}(35)$.GE. 1.0E-7)) IFLG=29

if (IFLG.GE. 1) go to 607 ! $36=$ PCGSO

if $((\operatorname{chip}(36)$.LE. 0.0) .OR. ( $\operatorname{chip}(36)$.GE. 1.OE-7)) IFLG=30

if (IFLG.GE. 1) go to 607 ! $37=$ PCGBO

if ((chip(37) .LE. 0.0) .OR. ( $\operatorname{chip}(37)$.GE. 1.0E-7)) IFLG=31

if (IFLG .GE. 1) go to 607 ! $38=$ PERR

if $((\operatorname{chip}(38) . \mathrm{LT}, 0.0)$.OR. ( $\operatorname{chip}(38) . \mathrm{GT}, 10.0)) \quad \mathrm{IFLG}=31$ 


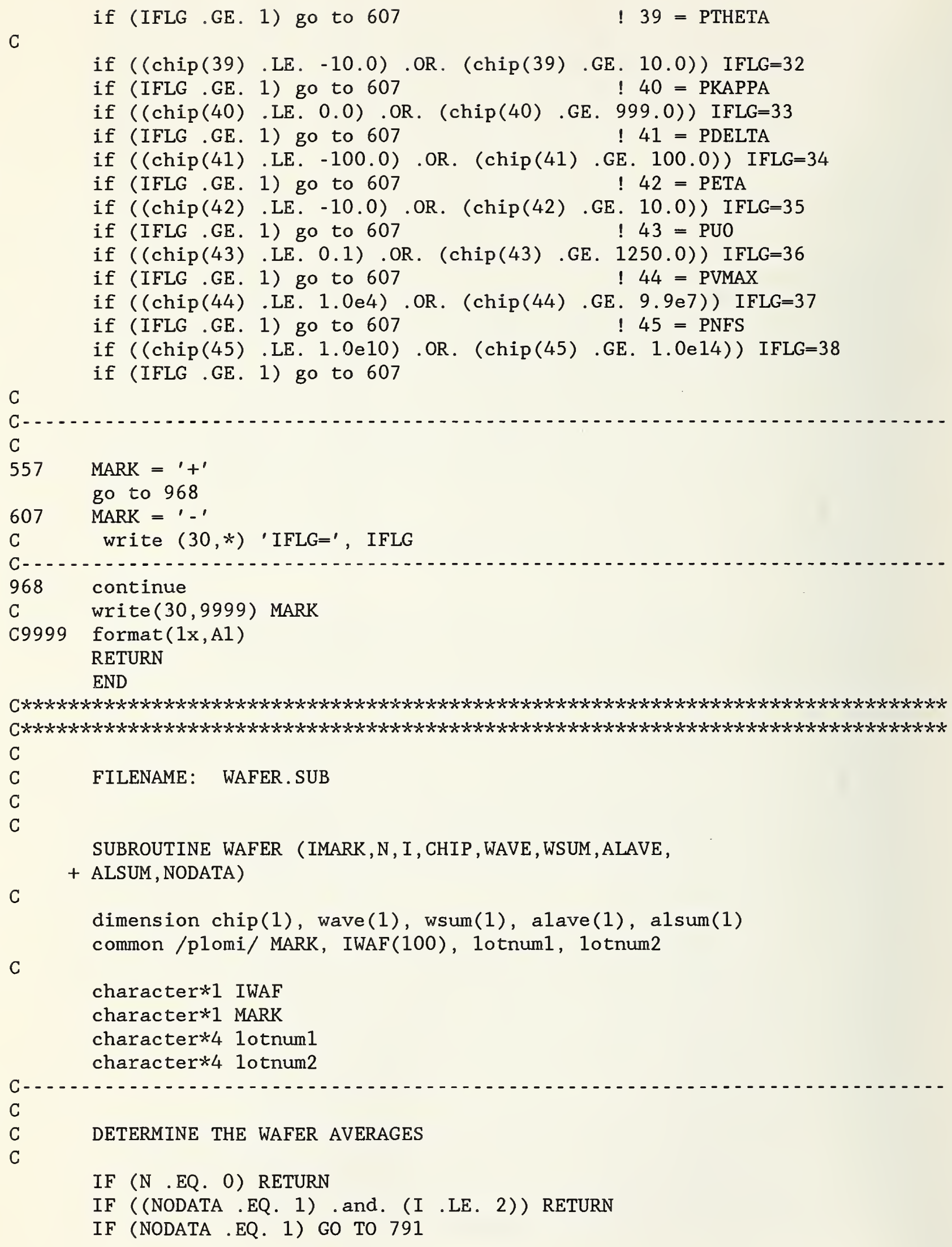


IF (I .EQ. 1) GO TO 201

IF (IWAF(I) .NE. IWAF(I-1)) GO TO 309

201 if (imark.1e. 0) go to 309

$\mathrm{J}=0$

DO $200 \mathrm{~J}=1,47$

$\operatorname{WSUM}(\mathrm{J})=\operatorname{WSUM}(\mathrm{J})+\operatorname{CHIP}(\mathrm{J})$

$\operatorname{WAVE}(\mathrm{J})=\operatorname{WSUM}(\mathrm{J}) / \mathrm{N}$

200 CONTINUE

$\mathrm{C}$

C.

309 if (I .EQ. 1) RETURN

if (IWAF(I) .eq. IWAF(I - 1)) RETURN

791 WRITE $(30,4)$

WRITE $(30, *)$ ' $* * *$ WAFER AVERAGES $* * *$ '

WRITE $(30,4)$

WRITE $(30,4)$

WRITE $(30, *) ' *$ W1 $+{ }^{\prime}$

LP

$\operatorname{WRITE}(30, *), *$

$\operatorname{WRITE}(30, *)$ ' $*$ '

WRITE $(30,6)$

WRITE $(30,7)$

WRITE $(30,8)$

WRITE $(30,9)$

WRITE $(30,806) \mathrm{N}, \mathrm{I}-1$

806 format ( $1 \mathrm{x},{ }^{\prime}$ Num chips/Tot chips $\left.=^{\prime}, \mathrm{I} 2,{ }^{\prime} /{ }^{\prime}, \mathrm{I} 2\right)$

C

$\operatorname{WRITE}(30,46) \operatorname{IWAF}(I-1), \operatorname{WAVE}(1), \operatorname{WAVE}(2), \operatorname{WAVE}(46), \operatorname{WAVE}(47)$

WRITE $(30,48)$ WAVE $(3), \operatorname{WAVE}(4), \operatorname{WAVE}(5)$

WRITE $(30, *)$ ' '

inc $=0$

if (idev .eq. 2) inc $=20$ ! just $p$-channel

821 write $(30,42)$ wave $(15+$ inc), wave $(16+$ inc), wave $(17+i n c)$,

+ wave $(11+$ inc $)$

write $(30,43)$ wave $(9+i n c)$, wave $(8+i n c)$, wave $(10+i n c)$,

+ wave $(6+i n c)$, wave $(7+i n c)$

WRITE $(30,44)$ wave $(13+i n c)$, wave $(12+i n c)$, wave $(23+i n c)$,

+ wave $(21+i n c)$, wave $(24+i n c)$

WRITE $(30,45)$ wave $(14+i n c)$, wave $(20+i n c)$, wave $(25+i n c)$,

+ wave $(22+i n c)$, wave $(19+i n c)$, wave $(18+i n c)$

if ((idev .eq. 1) .or. (inc.ge. 20)) go to 972

write $(30,47)$

inc $=20$

go to 821

$\mathrm{C}$

C

C

4 format (' $\left.*^{\prime}\right)$

6 format $(1 *$

$7 \quad+$ format $\left({ }^{\prime} *\right.$ ')

CGDO CGSO CGBO',

LD

TOX

SUB ' , 


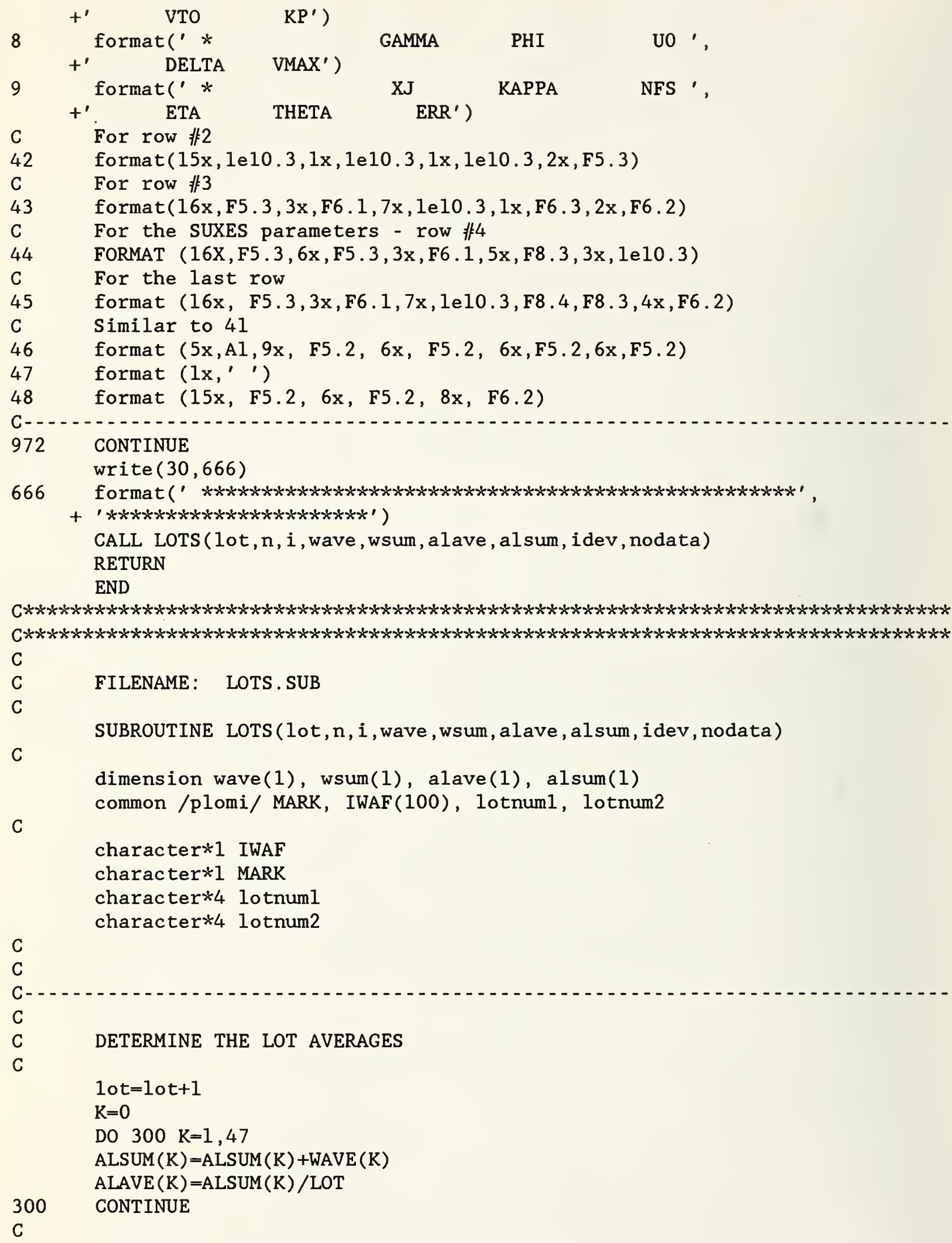


C REINITIALIZE THE WAFER DIMENSIONS

C

$\mathrm{N}=0$

$\mathrm{I}=0$

INC $=0$

DO 1000 INC $=1,47$

WAVE $($ INC $)=0.0$

WSUM ( INC) $=0.0$

1000 CONTINUE

IF (LOT .LT. 2) RETURN

IF (nodata .eq. 0) RETURN

C

C

C SUM UP THE RESULTS FOR THE LOT

C

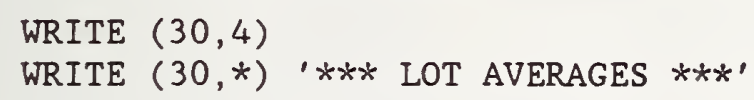

836 format ( $1 \mathrm{x},{ }^{\prime}$ Num wafers $\left.\mathbf{~}^{\prime}, \mathrm{I} 2\right)$

WRITE $(30,50)$ lotnum1, lotnum2, alave(1), alave(2), alave (46),

+ alave (47)

WRITE $(30,48)$ alave(3), alave(4), alave(5)

WRITE $(30, *), '$

inc $=0$

if (idev .eq. 2) inc $=20$ ! just $p$-channel

936 write $(30,42)$ alave $(15+i n c)$, alave $(16+i n c)$, alave $(17+i n c)$,

+ alave $(11+i n c)$

write $(30,43)$ alave $(9+i n c)$, alave $(8+i n c)$, alave $(10+i n c)$,

+ alave $(6+i n c)$, alave ( $7+i n c)$

WRITE $(30,44)$ alave $(13+i n c)$, alave $(12+i n c)$, alave $(23+i n c)$,

+ alave $(21+i n c)$, alave (24+inc)

WRITE $(30,45)$ alave (14+inc), alave (20+inc), alave (25+inc),

+ alave $(22+i n c)$, alave $(19+i n c)$, alave $(18+i n c)$

if ((idev .eq. 1) .or. (inc.ge. 20)) go to 937

write $(30,47)$

inc $=20$

go to 936

937 CONTINUE

write $(30,666)$

666 format (' $* * * * * * * * * * * * * * * * * * * * * * * * * * * * * * * * * * * * * * * * * * * * * * * * * * * * * *$ ', 


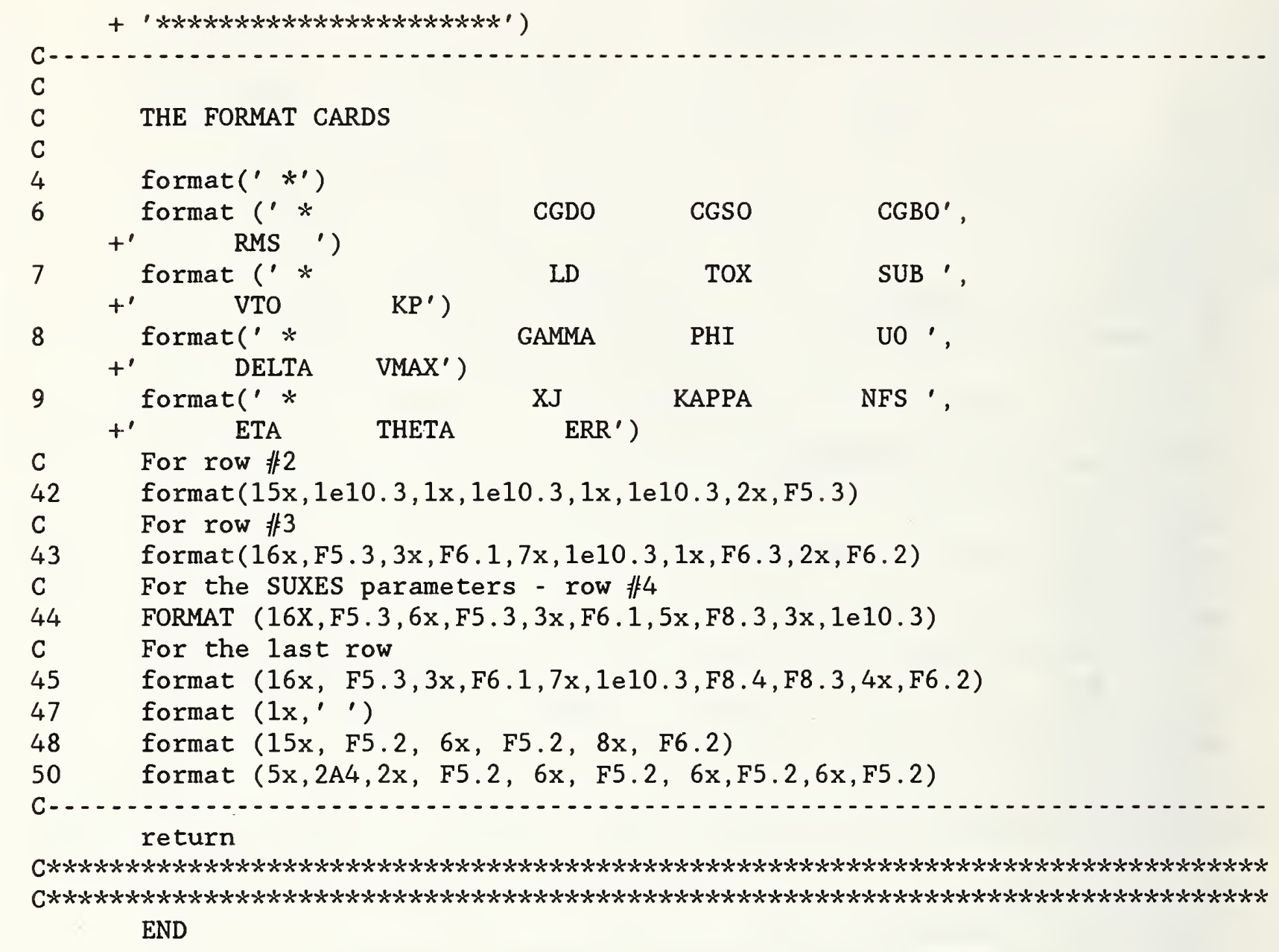




\section{RANKEY. FOR}

C

C

C

C

C

C

FILENAME: RANKEY.FOR

PURPOSE: THIS PROGRAM ORDERS THE CORRELATION COEFFICIENT DATA AND DETERMINES WHICH WAFER MAPS TO MAKE.

dimension numfile(50), filnl(50), filn2(50)

dimension number(50), $\operatorname{cor}(50)$, nchips(50)

dimension nummax(50), cormax(50), nchipmax(50)

C

character*1 iwaf

character*4 filn1

character*4 filn2

character $* 4$ filela

character*4 file $2 a$

character $* 4$ filelb

character*4 file $2 b$

character*4 lotnuml

character*4 lotnum2

C

open (unit $=10$, file='in.dat', status='old')

open (unit $=11$, file='lotnumber.fil', status='old')

open(unit=12, file='nlw.fil', status='old')

open (unit $=15$, file $=^{\prime}$ coref.fil', status='old')

open (unit $=20$, file='out.dat', status='unknown')

open (unit=30, file=' out2.dat', status=' unknown')

open (unit $=40$, file='mapl.dat', status='old')

C

open (unit $=50, \mathrm{file}=$ ' out4.dat', status='unknown')

C

C

C

read the lotnumber and wafer letter

read $(11,6)$ lotnum1, lotnum2

6 format (2A4)

read $(12,7)$ iwaf

7 format (Al)

C

C

read the coref file

C

$\mathrm{j}=0$

do $131 j=2,46$

read $(15,132$, end $=733)$ numfile(j), filnl(j), filn2(j)

132 format (I3, 2A4)

131 continue

C

C

read the mapl file

C

733 read $(40,833)$ filela, filelb

833 format (2A4)

read $(40,833)$ file $2 a$, file $2 b$

C 


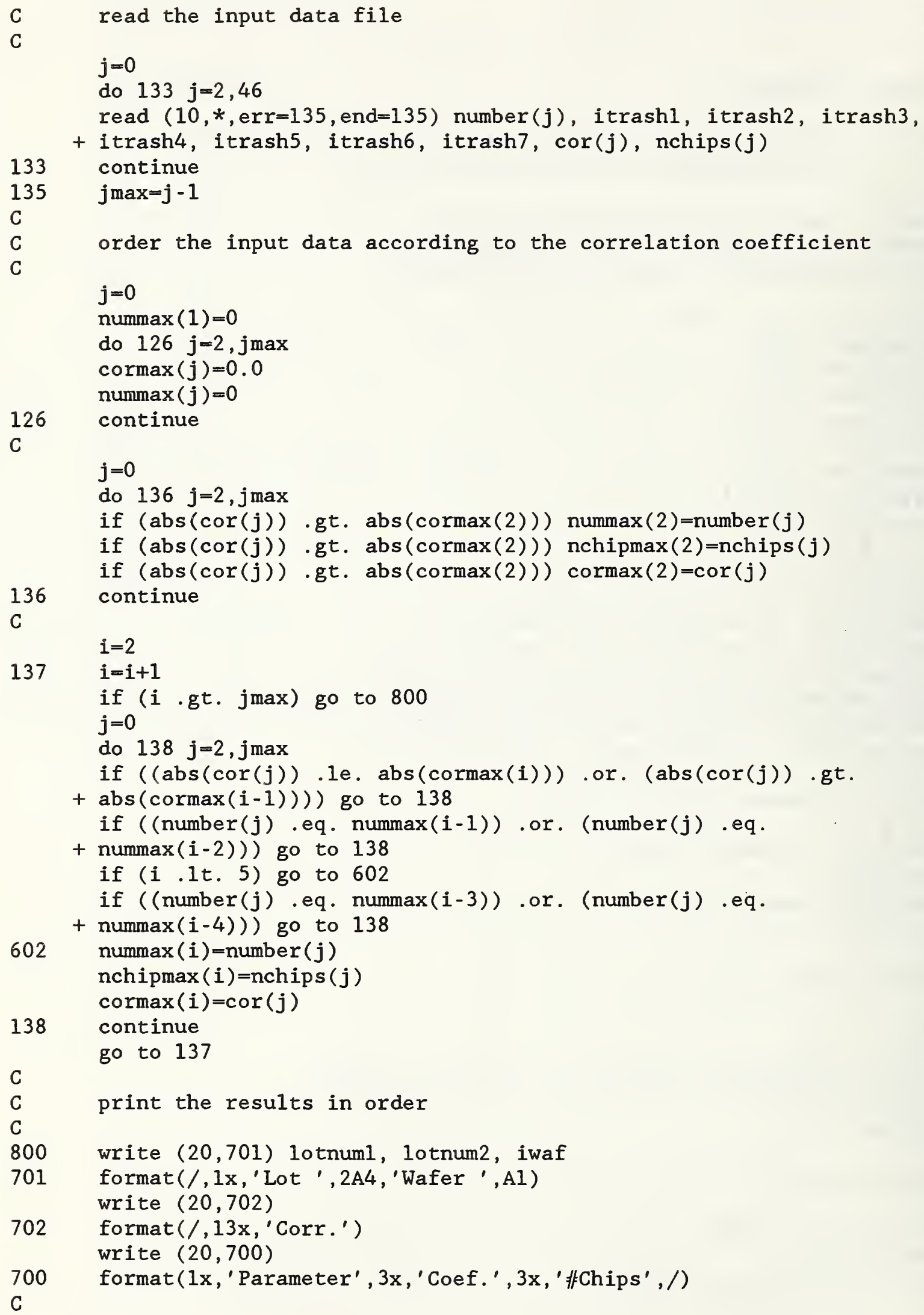




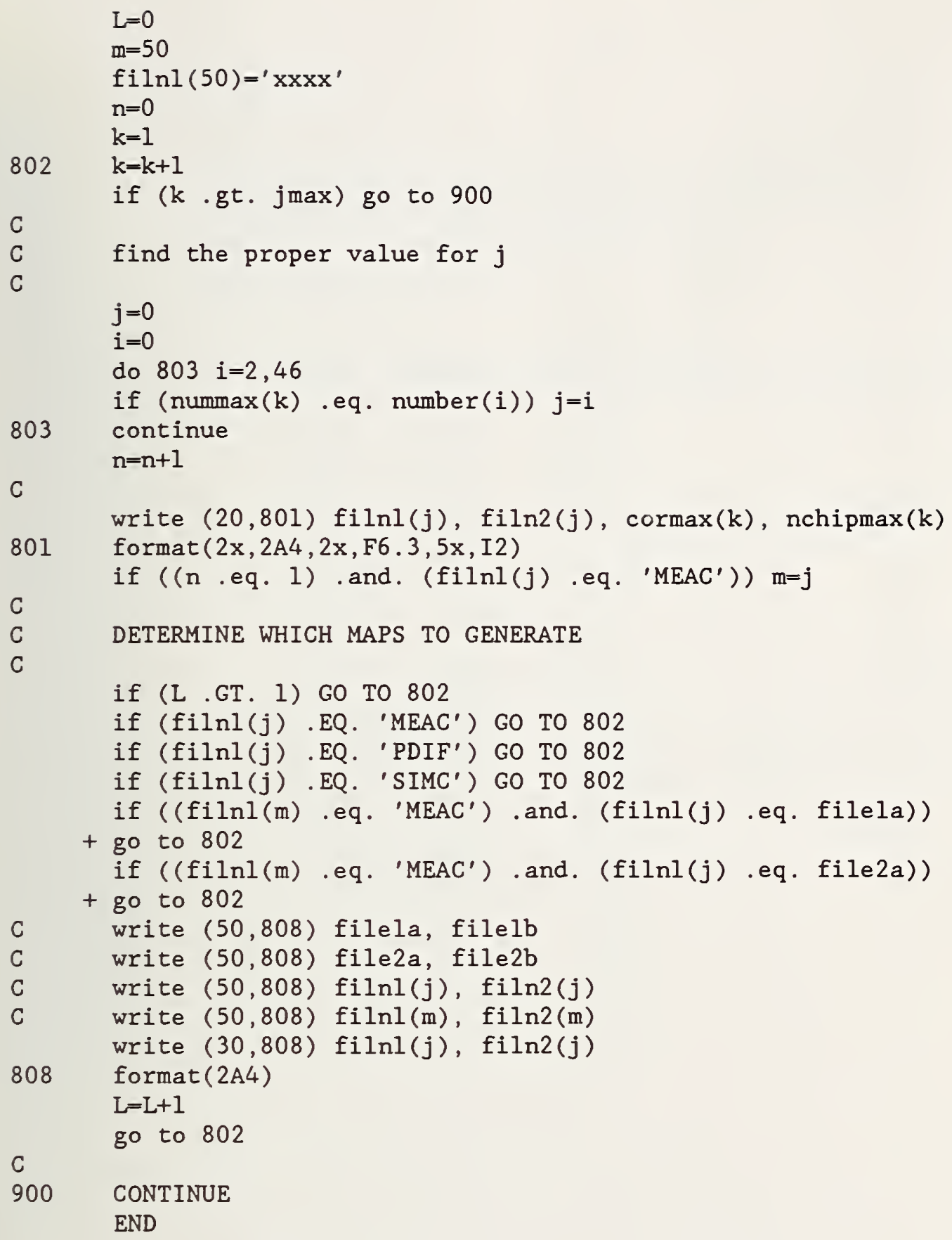




\section{NLW . FIL}

Y

0

4

2.46

2.46

RC.FIL

*

$*$ FILENAME $=$ RC.FIL

* THIS IS A FILE OF CHIPS OF WHICH SUXES AND SPICE ARE TO BE

* RUN

$*$

* Wafer Row Column

*

Y 24

LINE. FIL

*

$* \quad$ Filename $=$ LINE.FIL

* Xerox wafer

* Wafer Row Column Wn Ln Wp Lp (Measured Freq) NorPorBOTH

* FORMAT (2x, A1 , 2x, I2 , 1x, I2 , 1x, F5.2, 1x, F5.2, 1x, F5 . 2, 1x, F5.2, 1x, F5.2 , 1x, I3) $\mathrm{Y} \quad 2 \quad \begin{array}{lllllll}\mathrm{Y} & 2.46 & 2.46 & 6.46 & 2.46 & 9.40 & 3\end{array}$

LOTNUMBER. FIL

YY027

NPARIN . FIL

TOX LD

635.

.38

PPARIN.FIL

TOX LD

635 .

.45 


\section{NINPUT . XXX}

$*$

* FILENAME: NINPUT.DAT

$*$

* PURPOSE: THIS IS THE N-CHANNEL INPUT DATA FOR SPICE

* WHICH GIVES AN INITIAL GUESS AND THE RANGE IN WHICH TO EXPECT THE PARAMETER VALUE. SUXES IS RESTRICTED TO THIS RANGE.

Date generated 6-20-83

* parameters initial lowbound upperbound

\section{Level}

Type

XId

Tox

Nsub

Vto

$\mathrm{Xkp}$

Gamma

Phi

Uo

Uexp

Ucrit

Delta

$V \max$

$\mathrm{Xj}$

Lamda

Kappa

Nfs

Neff

Nss

Tgate

Eta

Theta
3

1.0

UUU

TTT

SSS

RRR

OOOd- 6

MMM

JJJ

KKK

0.0

0.0

2.685

$7.9 \mathrm{~d} 5$

HHH

0.0

.8000

2. $28 \mathrm{~d} 12$

0.0

lell

1.0

$.1306-2.0$

$.3612-2.0$

.1

0.0

0.0

0.0

0.0
3

1.0

1.0

1000.0

1. d17

$\begin{array}{ll}0.3 & 1.5\end{array}$

.12

$.3 \quad .9$

$0.1 \quad 1250.0$

$0.001 \quad 2.3$

1. d4 $1 . \mathrm{d} 6$

$-1 . d 2 \quad 1 . d 2$

1. $\mathrm{d} 4$

1.d-6

1.d-2

9. $9 \mathrm{~d} 7$

1.5

1.d-1

999.0

$4 \mathrm{e} 14$

1.d1

1 e14

1.0

2.0

2.0 
PINPUT . XXX

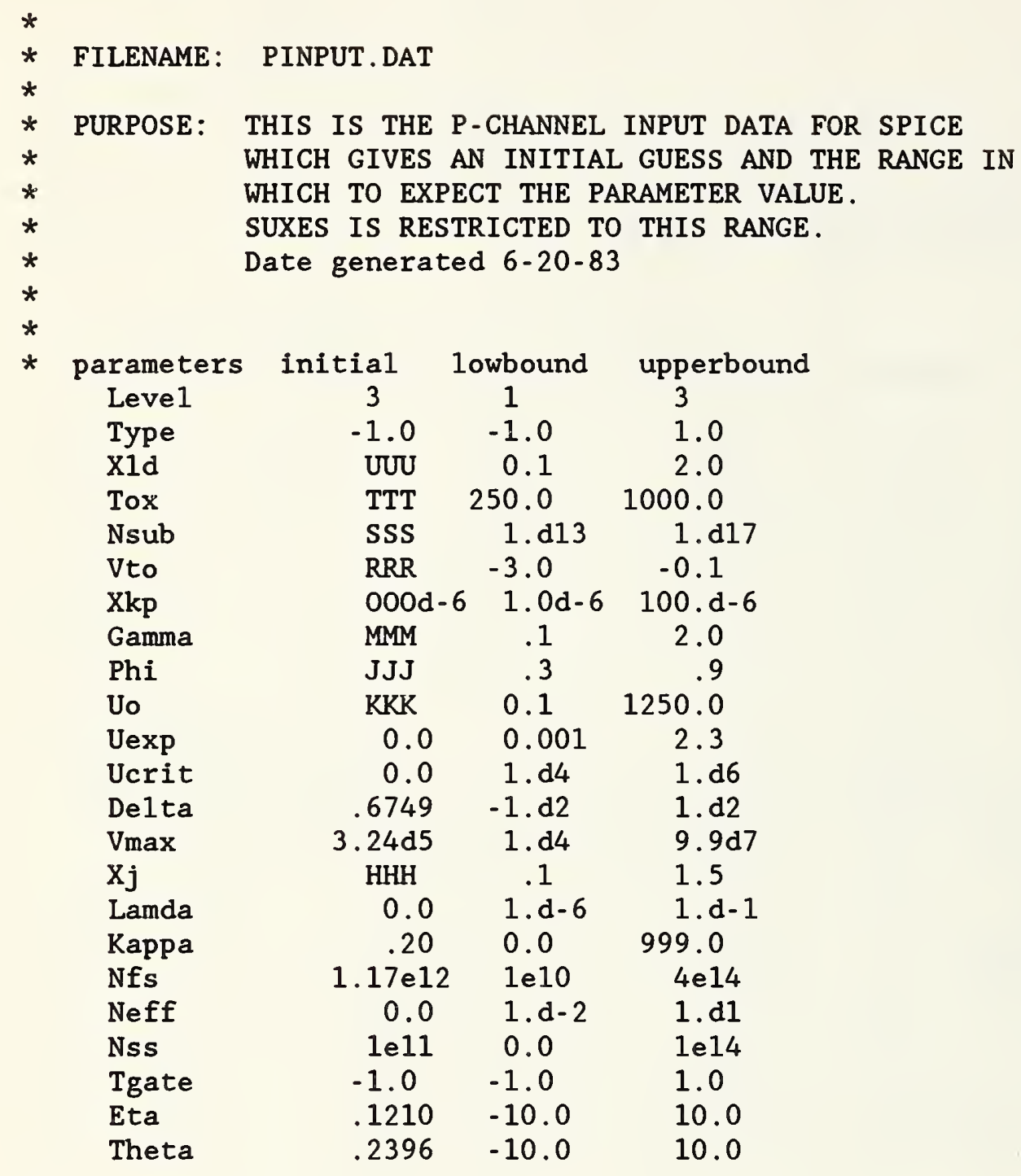


NOPT . FIL

*

* FIlename: NOPT.Fil

*

*

Criteria

Nsign=4 Maxfn=200 Delta=1.0d-9 Epsil=1.0d-6

Linit=0.1 Lscal=2.0 Luppr=1000 Fcdsw=0.1

$*$

RangVD $\min =0.00 \quad \max =5.5$ incr $=0.0$

RangVG $\min =0.00 \quad \max =5.5$ inc $r=0.0$

RangVB $\min =0.0 \quad \max =0.0$ inc $r=1.0$

Weight

POPT.FIL

*

*

$*$

$*$

*

*

$\star$

Criteria

Nsign=4 Maxfn=200 Delta=1.0d-9 Epsil=1.0d-6

Linit $=0.1$ Lscal=2.0 Luppr=1000 Fcdsw=0.1

RangVD $\min =-5.5 \quad \max =0.00$ inc $r=0.0$

RangVG $\min =-5.5 \max =0.00$ incr $=0.0$

RangVB $\min =0.0 \quad \max =0.0$ inc $r=1.0$

Weight 
NSTRAT . FIL

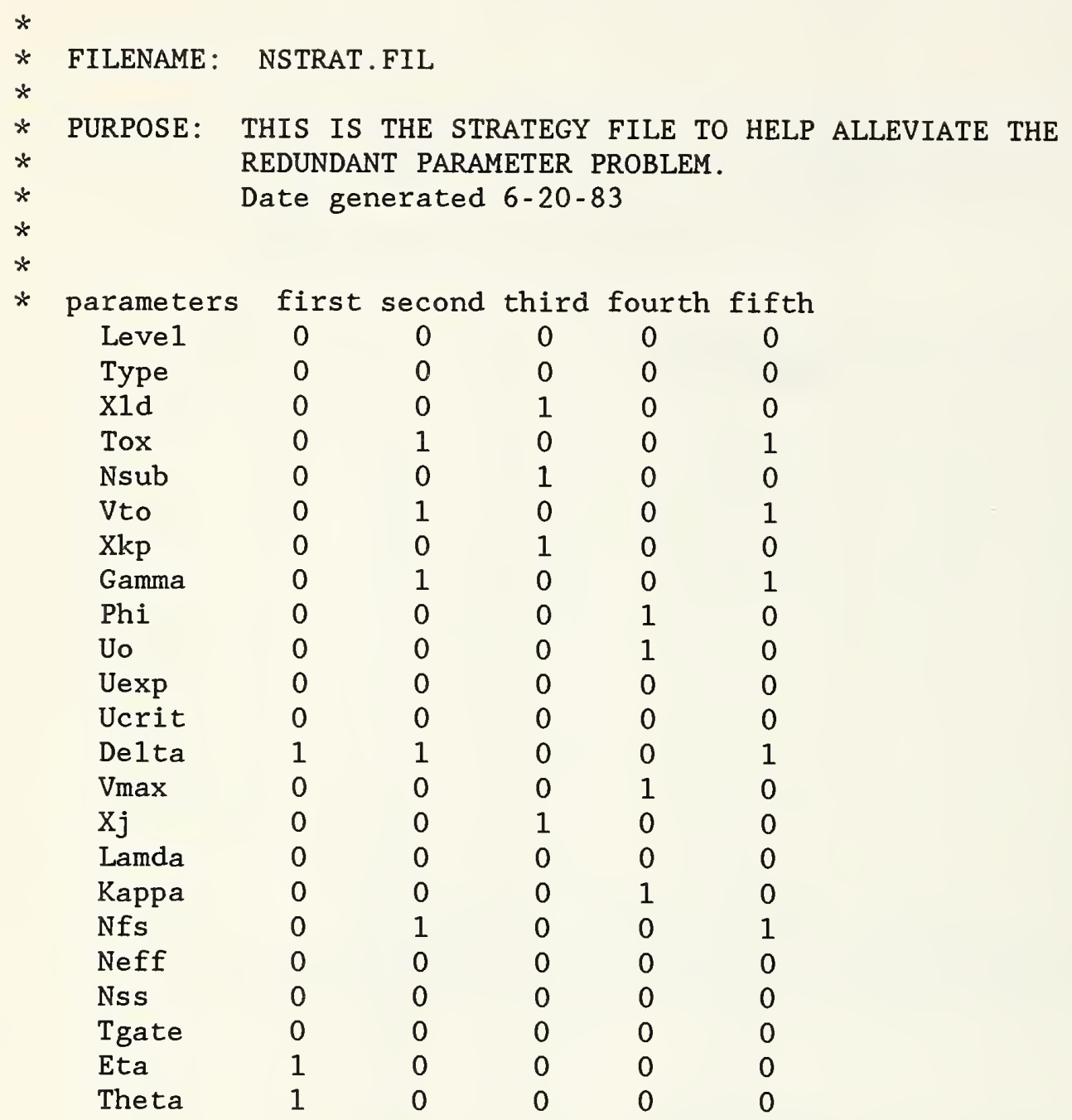


PSTRAT.FIL

* FILENAME: PSTRAT.FIL

* PURpose: this is the strategy file to help alleviate the

* REDUNDANT PARAMETER PROBLEM.

* $\quad$ Date generated 6-20-83

* parameters first second third fourth fifth

$\begin{array}{llllll}\text { Level } & 0 & 0 & 0 & 0 & 0 \\ \text { Type } & 0 & 0 & 0 & 0 & 0 \\ \text { Xld } & 0 & 0 & 1 & 0 & 0 \\ \text { Tox } & 0 & 1 & 0 & 0 & 1 \\ \text { Nub } & 0 & 0 & 1 & 0 & 0 \\ \text { Veto } & 0 & 1 & 0 & 0 & 1 \\ \text { Xkp } & 0 & 0 & 1 & 0 & 0 \\ \text { Gamma } & 0 & 1 & 0 & 0 & 1 \\ \text { Phi } & 0 & 0 & 0 & 1 & 0 \\ \text { Un } & 0 & 0 & 0 & 1 & 0 \\ \text { Uexp } & 0 & 0 & 0 & 0 & 0 \\ \text { Ucrit } & 0 & 0 & 0 & 0 & 0 \\ \text { Delta } & 1 & 1 & 0 & 0 & 1 \\ \text { Vax } & 0 & 0 & 0 & 1 & 0 \\ \text { Xi } & 0 & 0 & 1 & 0 & 0 \\ \text { Lamia } & 0 & 0 & 0 & 0 & 0 \\ \text { Kappa } & 0 & 0 & 0 & 1 & 0 \\ \text { Nf } & 0 & 1 & 0 & 0 & 1 \\ \text { Kef } & 0 & 0 & 0 & 0 & 0 \\ \text { Ns } & 0 & 0 & 0 & 0 & 0 \\ \text { Tgate } & 0 & 0 & 0 & 0 & 0 \\ \text { Eta } & 1 & 0 & 0 & 0 & 0 \\ \text { Theta } & 1 & 0 & 0 & 0 & 0\end{array}$

117 


\section{IVCHARN . XXX}

*

*

* FILENAME = IVCHARN.FIL

* NOTE: Use with MODEL.FIL to make IVCHARN.CEL

*

* N-CHANNEL MOS OUTPUT CHARACTERISTICS

*

M1 1200 MODN L=BZBUM W=FFFUM $A D=12.5 P \quad A S=12.5 P \quad P D=15 U$

$+\mathrm{PS}=15 \mathrm{U} \mathrm{NRD}=2.0 \mathrm{NRS}=2.0$

*

VD 30

VG 20

VIDS 31

*

.OPTIONS NODE NOPAGE

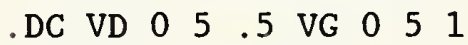

. PLOT DC I(VIDS)

.END

IVCHARP. XXX

*

*

* FILENAME $=$ IVCHARP. FIL

* NOTE: Use with MODEL.FIL to make IVCHARP.CEL

*

* P-CHANNEL MOS OUTPUT CHARACTERISTICS

$*$

M2 1200 MODP L=BZBUM W=FFFUM $A D=42.25 P \quad A S=42.25 P \quad P D=26 U$

$+\mathrm{PS}=26 \mathrm{U} \mathrm{NRD}=1.0 \mathrm{NRS}=1.0$

*

VD 40

VG 20

VIDS 41

*

.OPTIONS NODE NOPAGE

$\begin{array}{lllllll}\text {.DC VD } & 0 & -5 & -.5 \text { VG } & 0 & -5 & -1\end{array}$

.PLOT DC I(VIDS)

.END 


\section{NMODEL. XXX}

*

*

* FILENAME = NMODEL.FIL

* NOTE: This file uses parameters from the process and SUXES.

*

.MODEL MODN NMOS LEVEL $=3$ LD=sqsUM TOX=rqrUM NSUB=pqP VTO=oqoV

$+\mathrm{KP}=\mathrm{nqnU}$ GAMMA=mqm PHI=lql UO=kqk DELTA=jqj VMAX=iqi

$+\mathrm{XJ}=\mathrm{hqhUM} \mathrm{KAPPA}=\mathrm{gqg}$ NFS=fqf $E T A=$ eqe THETA=dqd

$+\mathrm{CJSW}=0.6 \mathrm{E}-9 \mathrm{CGDO}=\mathrm{cqc} \quad \mathrm{CGSO}=\mathrm{bqb} \mathrm{CGBO}=\mathrm{aqa}$

$+\mathrm{RSH}=11.63 \mathrm{JS}=5.0 \mathrm{E}-5 \quad \mathrm{TPG}=1.0 \mathrm{MJ}=.5 \mathrm{MJSW}=.5 \mathrm{~PB}=.9 \mathrm{XQC}=.45$

*

\section{PMODEL.XXX}

*

$*$

* FILENAME $=$ PMODEL.FIL

* NOTE: This file uses parameters from the process and sUXES. *

.MODEL MODP PMOS LEVEL=3 $L D=s q s U M$ TOX=rqrUM NSUB=pqp VTO=oqoV

$+K P=n q n U$ GAMMA $=m q m$ PHI $=1 q 1$ UO $=k q k$ DELTA $=j q j$ VMAX $=i q i$

$+\mathrm{XJ}=$ hqhUM $\mathrm{KAPPA}=\mathrm{gqg}$ NFS=fqf $\mathrm{ETA}=$ eqe THETA=dqd

$+\mathrm{CJSW}=0.6 \mathrm{E}-9 \quad \mathrm{CGDO}=\mathrm{cqc} \quad \mathrm{CGSO}=\mathrm{bqb} \quad \mathrm{CGBO}=\mathrm{aqa}$

$+\mathrm{RSH}=61.57 \mathrm{JS}=5.0 \mathrm{E}-5 \mathrm{TPG}=-1.0 \mathrm{MJ}=.5 \mathrm{MJSW}=.5 \mathrm{~PB}=.9 \mathrm{XQC}=.45$

*

SUBCKT . XXXX

$*$

*

* FILENAME $=$ INVERT.FIL

*

.SUBCKT INVERT 123

*

M1 100100 MODN L=hzhUM W=iziUM $A D=12.5 P \quad A S=12.5 P \quad P D=15 U$

$+\mathrm{PS}=15 \mathrm{U} \mathrm{NRD}=2.0 \mathrm{NRS}=2.0$

M2 100133 MODP $L=j z j U M \quad W=k z k U M \quad A D=42.25 P \quad A S=42.25 P \quad P D=26 U$

$+\mathrm{PS}=26 \mathrm{U} \mathrm{NRD}=1.0 \mathrm{NRS}=1.0$

R1 10021332

C1 $200.043 \mathrm{P}$

*

. ENDS INVERT

* 


\section{CIRCUIT.FIL}

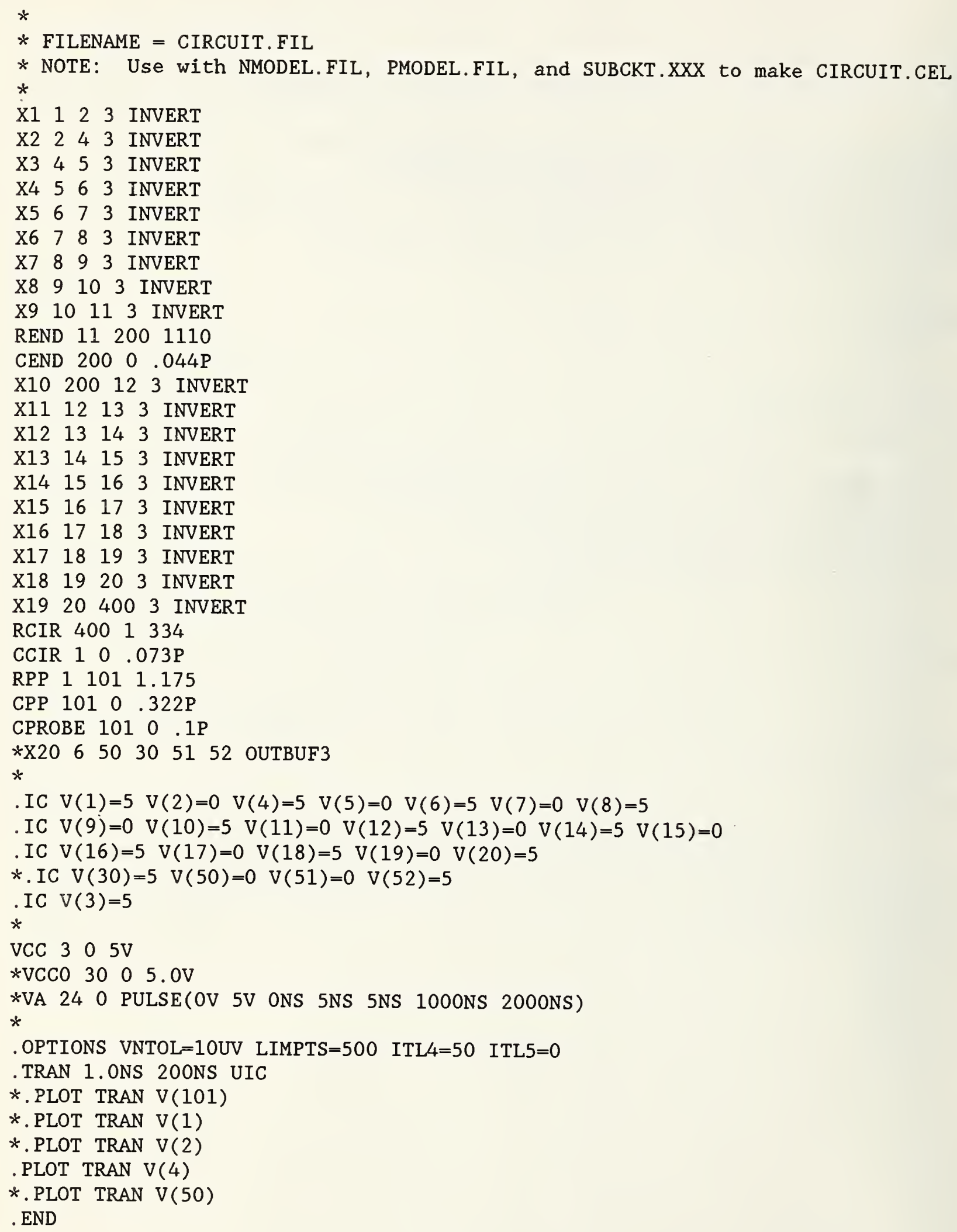


PCHIPS.FIL

* percent acceptable chips to make wafer maps... number of test sites/wafer 30.0 65

STEND. FIL

$\begin{array}{llll}4 & 2 & 1 & 1 \\ 6 & 8 & 9 & 9 \\ 1 & 1 & 1 & 2 \\ 9 & 9 & 9 & 8 \\ 4 & 0 & 0 & 0 \\ 6 & 0 & 0 & 0 \\ 0 & 0 & 0 & 0 \\ 0 & 0 & 0 & 0 \\ 0 & 0 & 0 & 0 \\ 0 & 0 & 0 & 0 \\ 0 & 0 & 0 & 0 \\ 0 & 0 & 0 & 0 \\ 0 & 0 & 0 & 0 \\ 0 & 0 & 0 & 0 \\ 0 & 0 & 0 & 0 \\ 0 & 0 & 0 & 0\end{array}$




\section{COREF . FIL}
$2 \mathrm{~W}$
$3 \mathrm{~L}$
4 WP
5 LP
6 MCKT
7 SCKT
8 PDIFF
9 NCGDO
10 NCGBO
11 NRMS
12 NLD
13 NTOX
14 NSUB
15 NVTO
16 NKP
17 NGAMMA
$18 \mathrm{NPHI}$
19 NUO
20 NDELTA
21 NVMAX
22 NXJ
23 NKAPPA
24 NNFS
25 NETA
26 NTHETA
27 NERR
28 PCGDO
29 PCGBO
30 PRMS
31 PLD
32 PTOX
33 PSUB
34 PVTO
35 PKP
36 PGAMMA
37 PPHI
38 PUO
39 PDELTA
40 PVMAX
41 PXJ
42 PKAPPA
43 PNFS
44 PETA
45 PTHETA
46 PERR 


\section{EDNO. WAY}

!

! filename = edno. way

!

delete 1:end-1

exit

EDSUXN. WAY

$!$

! filename $=$ EDSUXN. WAY

!

$\mathrm{s} / 77 / \mathrm{N} / 1$ : end

s/22/N22/1:end

exit

EDSUXP . WAY

!

! filename $=$ EDSUXP.WAY

!

s/77/P/1 : end

$s / 22 / P 22 / 1$ : end

exit 


\section{EDPAR . WAY}

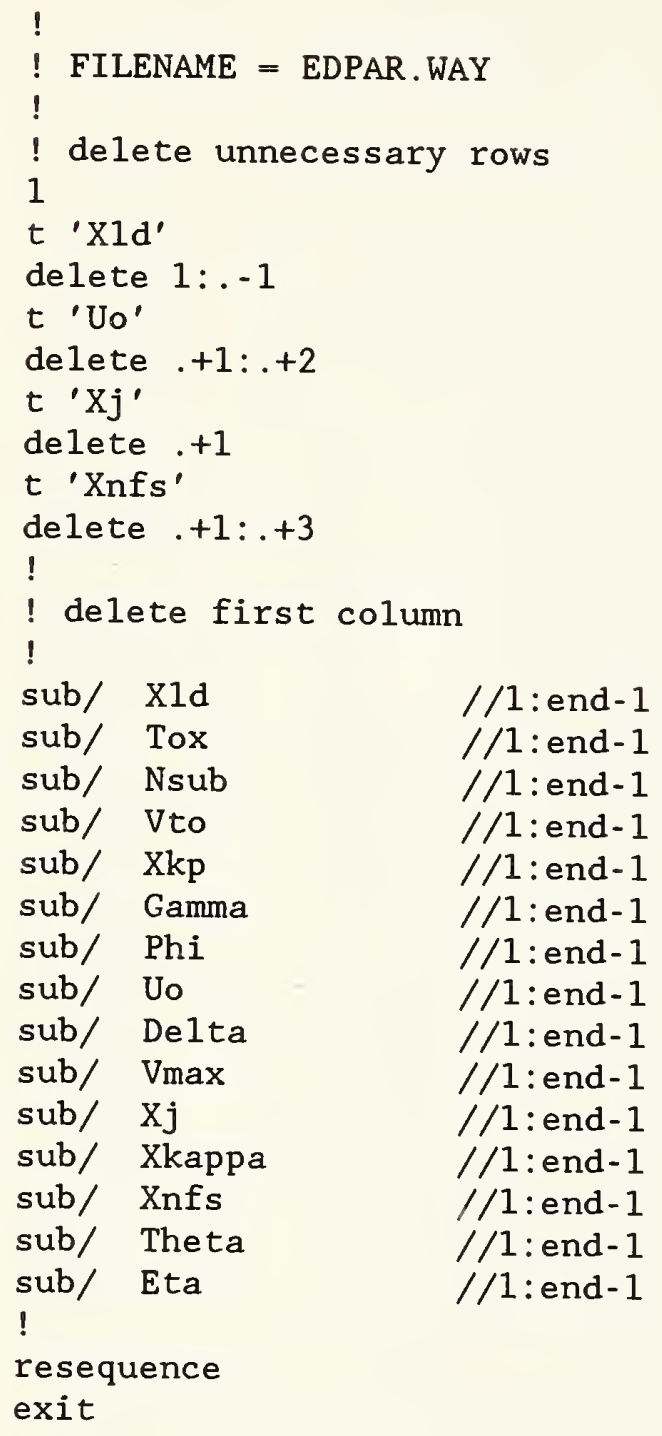


EDSPICEN. WAY

$!$

$!$ FILENAME $=$ EDSPICEN. WAY

!

s/ //end - 5 :end

$s / 777 / \mathrm{N} / 1$ : end

exit

EDSPICEP . WAY

!

! FILENAME $=$ EDSPICEP.WAY

!

s/ $/ /$ end- 5 :end

$\mathrm{s} / 777 / \mathrm{P} / 1$ : end

exit

EDLAST . WAY

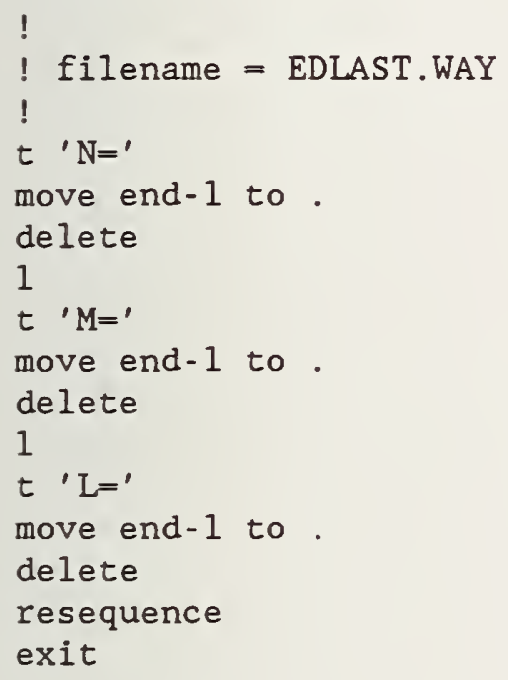




\section{EDNRMS . WAY}

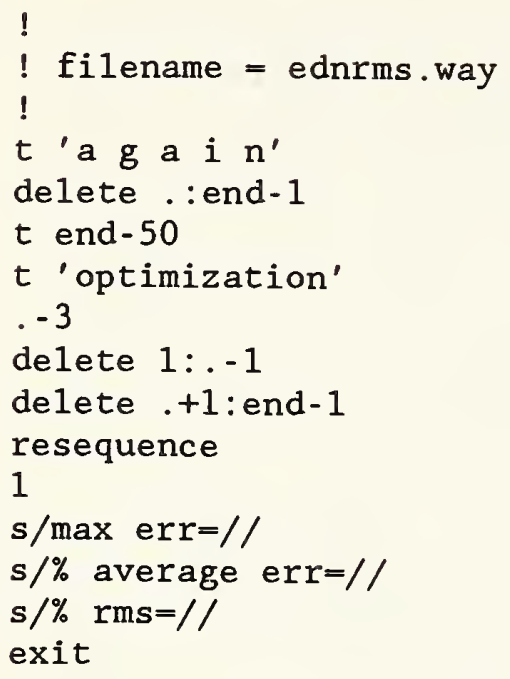

\section{EDRES . WAY}

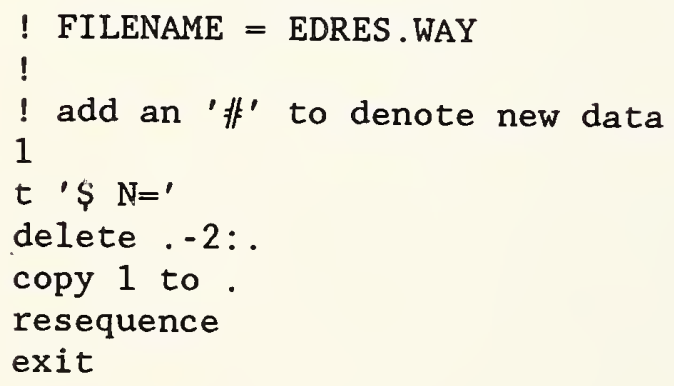




\section{EDSKEY.WAY}

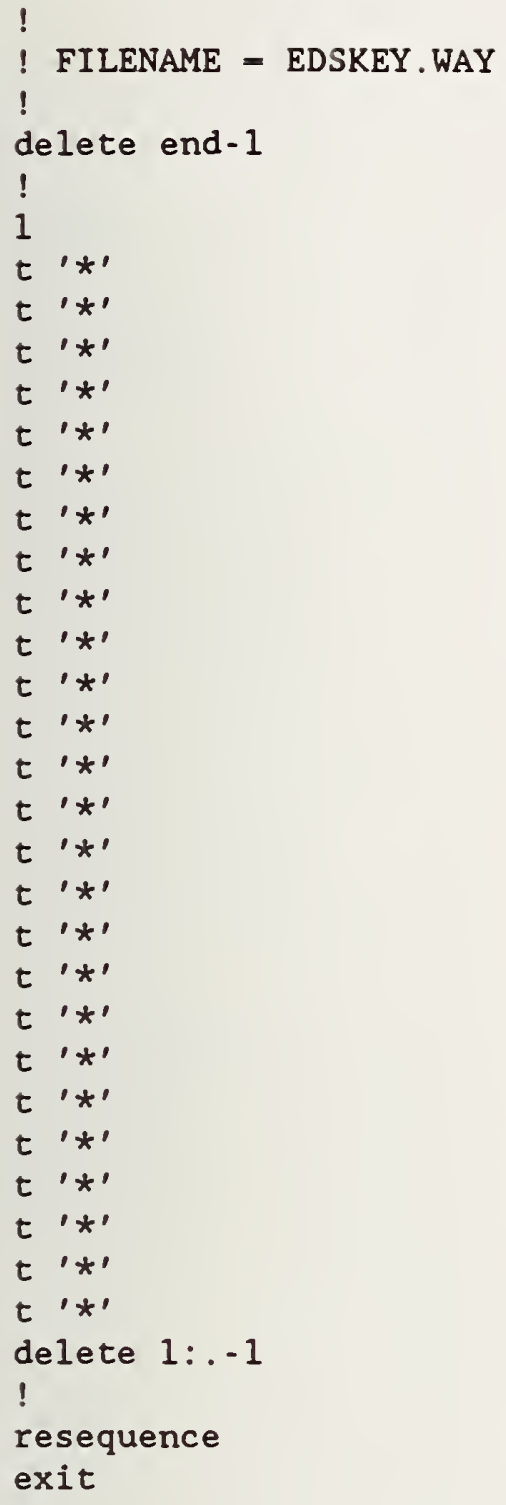


EDMAP . WAY

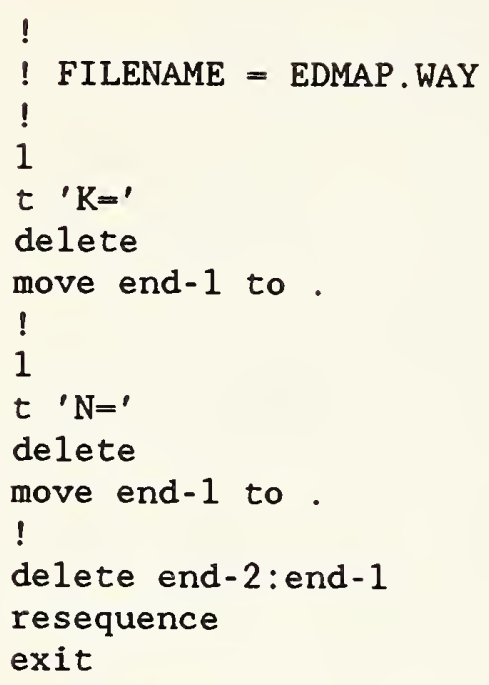


EDPDIFF . WAY

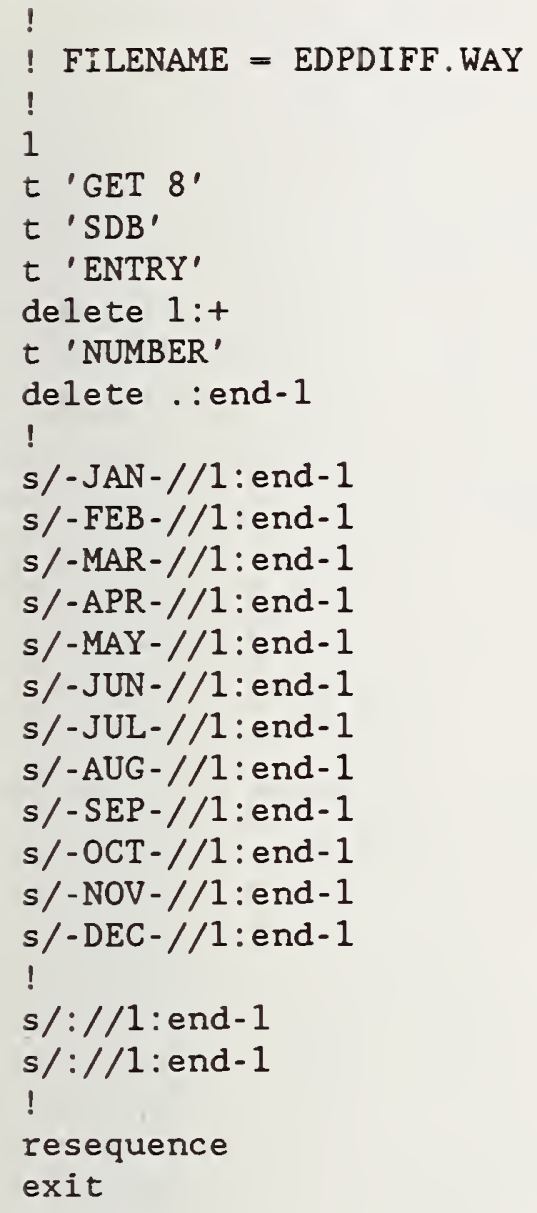




\section{EDMEAF . WAY}

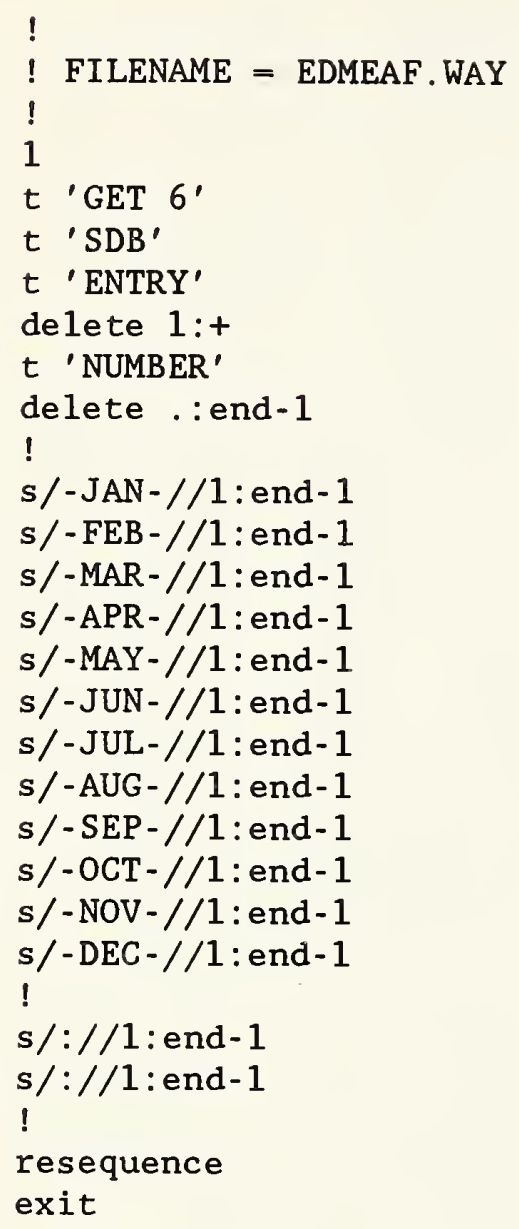




\section{Appendix B - Modifying SUXES's Strategy File}

The strategy file needs modification if: (1) SUXES's predicted I-V characteristics do not resemble the measured I-V characteristics, (2) the user does not accept the percent deviation between these curves, and (3) the user is not satisfied with the percent difference between the simulated and measured ring oscillator frequencies. One such modification technique is to determine the parameter(s) which is(are) causing the deviation in the I-V characteristics. To do this, plot the measured I-V data along with the I-V characteristics using the initial estimates for the parameter values. If this plot is not qualitatively satisfactory to the user, the values for the initial estimates must be reexamined. If using the initial estimates for the parameter values gives I-V characteristics which resemble the measured I-V characteristics, then plot the I-V characteristics after the values of the first iteration of parameters are optimized.

If the user accepts these plots, continue until an iteration of parameters fails to produce an acceptable plot. In the set of parameters which produces the unsatisfactory plot, eliminate one parameter at a time in the strategy file to determine which one(s) cause(s) the deviation between the measured I-V data and the simulated I-V data. By using this method, the parameter(s) causing the deviation can be found. The parameter KAPPA (the saturation field factor) is an example of the above. Assume the user is not satisfied with the upward bending of the I-V characteristics in the saturation region, and assume KAPPA is causing this bending after the first iteration, while the I.V characteristics using the initial estimates do not have this trait. A technique to eliminate the effect of KAPPA is to obtain an accurate initial estimate of the value of this parameter and then avoid optimizing this parameter's value until the values of the other parameters have been optimized. To obtain an initial estimate for the value of KAPPA, a parameter value must be optimized which has very little influence on the result (for example, the value of NFS). Therefore, using about four or five different initial estimates for the value of KAPPA, SUXES can be run to optimize only the value of NFS while using the initial estimates for the values of the other parameters. An appropriate value of KAPPA is found when the user is satisfied with the resemblance of the simulated I-V characteristics to the measured I-V data. Using the technique described above, the simulated I-V characteristics will have a realistic shape even though the rms deviation might be lower with other, less realistic optimization techniques.

After a reasonable initial estimate for the value of KAPPA is found, an appropriate strategy file must be found. SUXES can operate in an automated or a manual mode. Up until now it did not matter in which mode it was operating. At this point, it is best to use the manual mode which includes a sensitivity analysis. To obtain an appropriate strategy file, several parameters (perhaps three) can be randomly selected and optimized. These are potential candidates for the first iteration of parameters. These parameters can be optimized as a group if no errors occur and high values in the sensitivity analysis are obtained. Low values in the sensitivity analysis indicate the existence of redundant parameters, and the directions of the eigenvectors indicate which parameters are redundant $[1,16]$. The boundary values may be altered to decrease the number of errors due to the 
pegging of a parameter at a boundary. If the above criteria have been met, and if a plot of the measured I-V data resembles the simulated I-V data, then the first three parameters have been found.

After the values of the first three parameters are found, the process is repeated for the second, third, etc., iteration of parameters until the values of all the parameters are optimized and the user is satisfied with the rms deviation. The values of the parameters can be optimized more than once.

The optimization technique for the p-channel MOSFETs, which follows, was found in the manner described above. This optimization technique is also used for the $n$-channel MOSFETs.

(i) DELTA, ETA, THETA (the first iteration parameters)

(ii) TOX, VTO, GAMMA, DELTA, NFS (the second iteration parameters, etc.)

(iii) LD, NSUB, KP, XJ

(iv) PHI, UO, VMAX, KAPPA

(v) TOX, VTO, GAMMA, DELTA, NFS 


\section{Appendix C - The Procedure to Run KEYS}

The computer procedure KEYS was written on a VAX-11/780 using VAX/VMS. Data are initially received from the ACCUTEST, then transferred to the VAX subdirectory which contains the computer procedure KEYS.

The ACCUTEST data files have a format similar to that shown in table C-1. On the line beginning with POS, the chip position is recorded. The following two lines are ignored. The data values are recorded on the remaining lines starting with VAL. First, the $\mathrm{V}_{\mathrm{ds}}$ value is listed, followed on consecutive lines by the $V_{g s}$ value, the $V_{b s}$ value, and then the $I_{d s}$ value. This sequence is repeated for the remainder of the data sets. KEYS converts these data into a format, as shown in table C-2, suitable for SUXES. Also, KEYS provides the plotting package, PLOTMY, with the $\mathrm{V}_{\mathrm{ds}}$ and $\mathrm{I}_{\mathrm{ds}}$ data points needed to plot the I-V characteristics. The format for the plot file is similar to that given in table C-3 (without the date and time). The other plotting package, S5PLOT, plots the ring oscillator node potential as a function of time and records the period and frequency. It is provided data similar to those shown in table C-3.

The files listed in the order of their occurrence for the computer procedure KEYS are given in table C-4. The files that may be edited before KEYS is run are given in table C-5. After editing, KEYS is evoked by typing "submit/noprinter/notify KEYS." 
Table C-1. Example of a Data File from the ACCUTEST

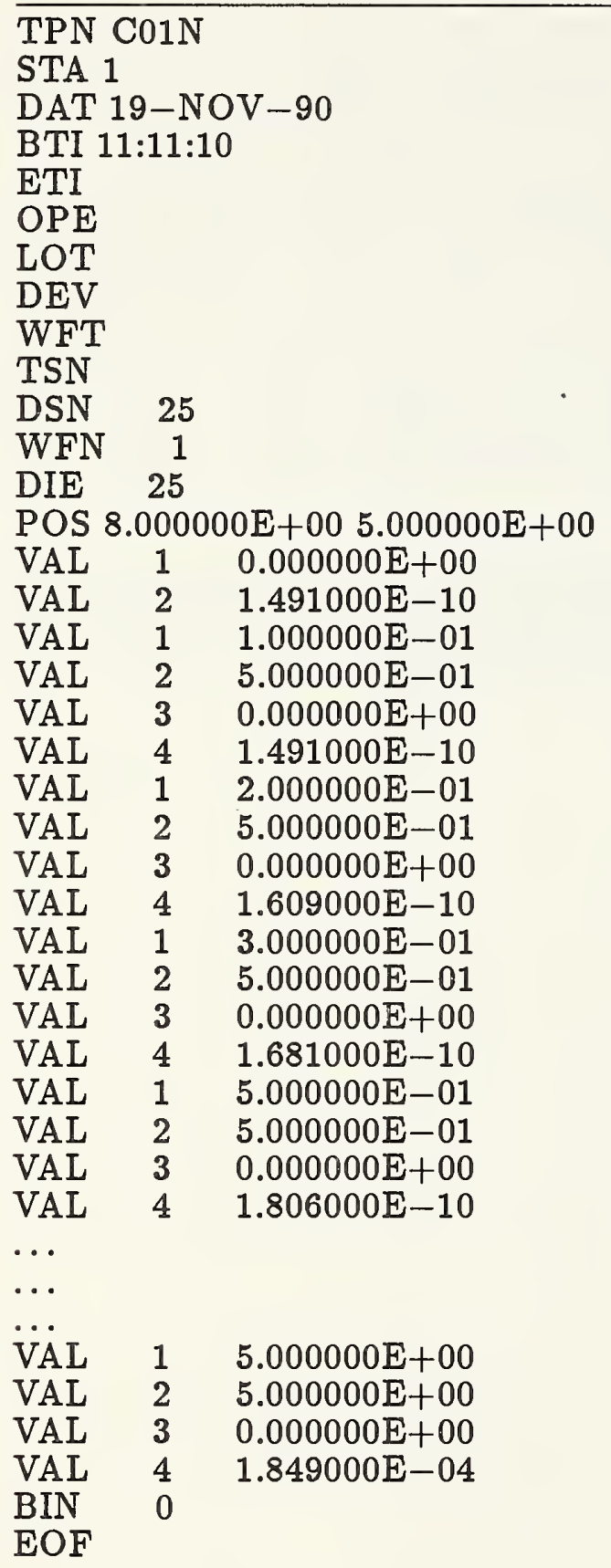


Table C-2. Example of a SUXES Data File

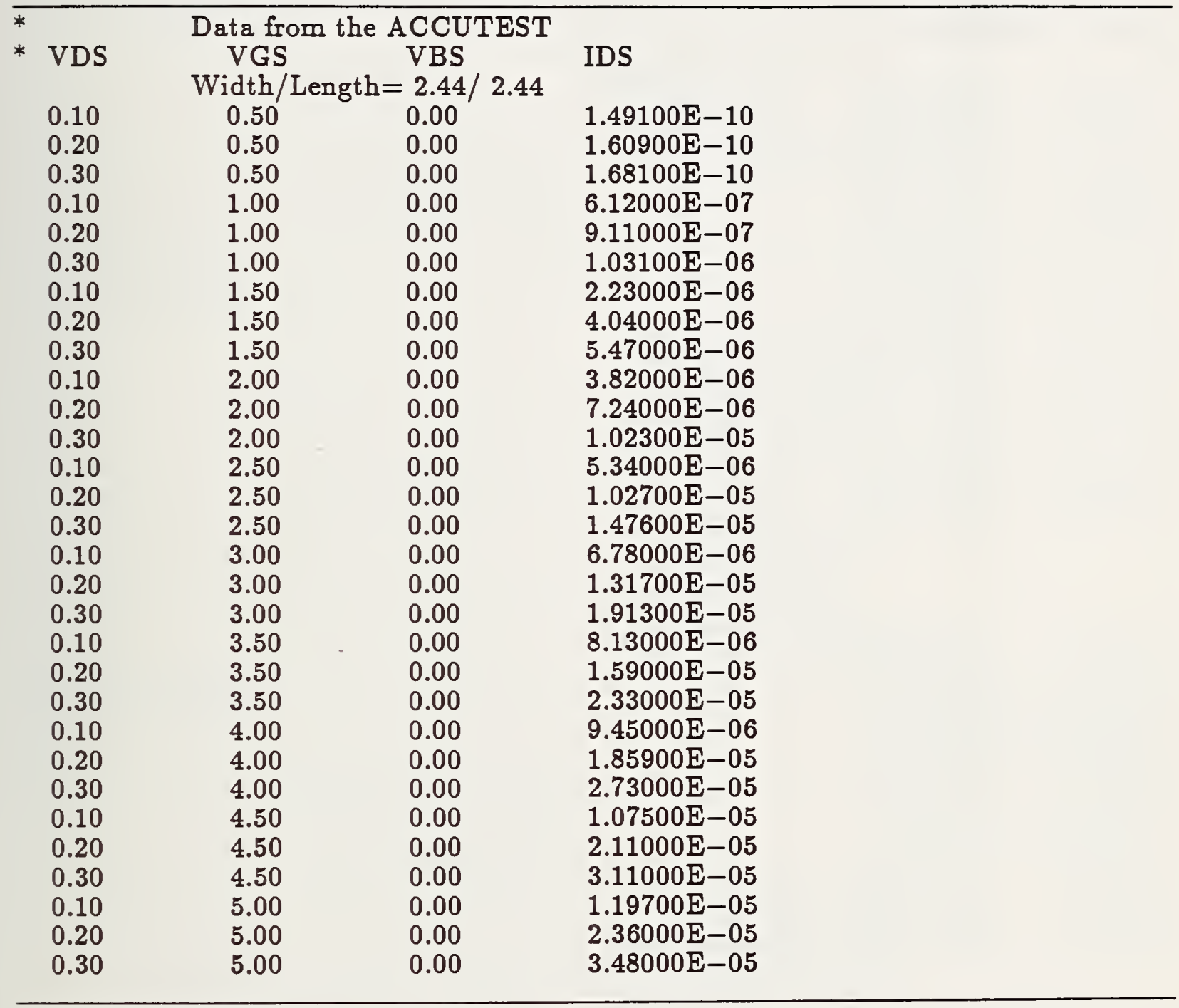


Table C-3. Example of a Plot File
19-NOV-90 05:15:55
201
$0.000 \mathrm{E}+00 \quad 1.158 \mathrm{E}+00$
$1.000 \mathrm{E}-09 \quad 6.697 \mathrm{E}-01$
$2.000 \mathrm{E}-09 \quad 5.665 \mathrm{E}-01$
$3.000 \mathrm{E}-09 \quad 4.710 \mathrm{E}-01$
$4.000 \mathrm{E}-09 \quad 3.861 \mathrm{E}-01$
$5.000 \mathrm{E}-09 \quad 3.147 \mathrm{E}-01$
$6.000 \mathrm{E}-09 \quad 2.560 \mathrm{E}-01$
$7.000 \mathrm{E}-09 \quad 2.082 \mathrm{E}-01$
$8.000 \mathrm{E}-09 \quad 1.683 \mathrm{E}-01$
$9.000 \mathrm{E}-09 \quad 1.362 \mathrm{E}-01$
$1.000 \mathrm{E}-08 \quad 1.101 \mathrm{E}-01$
$1.100 \mathrm{E}-08 \quad 8.813 \mathrm{E}-02$
$1.200 \mathrm{E}-08 \quad 7.098 \mathrm{E}-02$
$1.300 \mathrm{E}-08 \quad 5.754 \mathrm{E}-02$
$1.400 \mathrm{E}-08 \cdot 4.680 \mathrm{E}-02$
$1.500 \mathrm{E}-08 \quad 3.767 \mathrm{E}-02$
‥ $\quad \cdots$

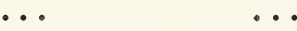
……
$1.900 \mathrm{E}-07 \quad 1.040 \mathrm{E}+00$
$1.910 \mathrm{E}-07 \quad 2.005 \mathrm{E}+00$
$1.920 \mathrm{E}-07 \quad 3.091 \mathrm{E}+00$
$1.930 \mathrm{E}-07 \quad 4.029 \mathrm{E}+00$
$1.940 \mathrm{E}-07 \quad 4.770 \mathrm{E}+00$
$1.950 \mathrm{E}-07 \quad 4.915 \mathrm{E}+00$
$1.960 \mathrm{E}-07 \quad 4.977 \mathrm{E}+00$
$1.970 \mathrm{E}-07 \quad 4.993 \mathrm{E}+00$
$1.980 \mathrm{E}-07 \quad 4.997 \mathrm{E}+00$
$1.990 \mathrm{E}-07 \quad 4.999 \mathrm{E}+00$
$2.000 \mathrm{E}-07 \quad 5.000 \mathrm{E}+00$ 
Table C-4. The Files Listed in the Order of Their Occurrence in the Computer Procedure KEYS. This list does not include files that are created during the program. The FORTRAN files are provided data files which can be edited by the editor files. The command procedures are connected together creating the computer procedure KEYS.

POSSIBLE

COMMAND

PROCEDURES
FORTRAN

FILES
DATA

FILES
EDITOR

FILES

(1) KEYS.COM

(1) RCKEY.FOR

+ (1) NLW.FIL

(1) EDNO.WAY

+ (2) RC.FIL

+ (3) LINE.FIL

+ (4) LOTNUMBER.FIL

(2) LAST3.COM

(3) INKEY.XXX

(2) SUKEY.FOR

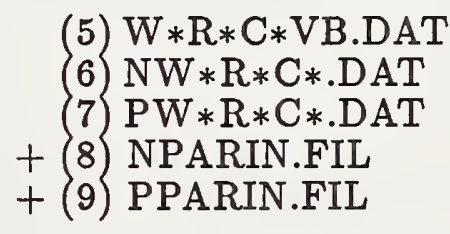

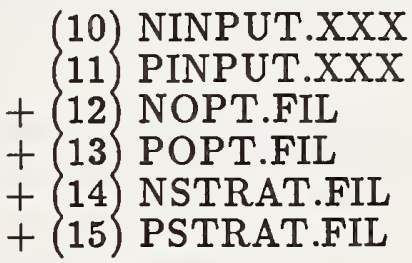

(3A) SUXES.XXX

* (3) SSUXES.FOR

(2) EDSUXN.WAY

(3) EDSUXP.WAY

(4) EDPAR.WAY

(4) CAPKEY.FOR

+ (16) IVCHARN.XXX

+ 17) IVCHARP.XXX

+18 NMODEL.XXX

+ (19) PMODEL.XXX

(3B) SPICEIV.XXX

(5) SPICENBS.FOR

(5) EDSPICEN.WAY

(6) EDSPICEP.WAY

6) IVKEY.FOR

(7) PLOTMY.FOR

$+(21)$ CIRCUIT.FIL 
(8) S5PLOT.FOR

(9) MCVAX.FOR

(4) RMSKEY.COM

(10) SUMKEY.FOR

$+(22)$ PCHIPS.FIL

(7) EDLAST.WAY

(8) EDNRMS.WAY

9) EDPRMS.WAY

(10) EDRES.WAY

(5) STKEY.COM

(11) CRDB.FOR

+ (23) STEND.FIL (11) EDSKEY.WAY

(12) STAT2.FOR

(6) CORKEY.XXX

(13) RANKEY.FOR

(24) COREF.FIL

(12) EDM.AP.WAY

(13) EDSIMF.WAY

(14) EDPDIFF.WAY

(15) EDMEAF.WAY

(7) MAPKEY.XXX

(16) EDMAPS.WAY

To obtain this program, consult K. Doganis. Refer to table 1 for details.

$+\quad$ The user may edit these files according to table C-5. 
Table C-5. The Files to be Edited at the Discretion of the User

\begin{tabular}{lll}
\hline FILENAME & POSSIBLE MODIFICATIONS & FORMAT
\end{tabular}

(1) NLW.FIL

(2) RC.FIL

(3) LINE.FIL

(6) PPARIN.FIL

(7) NOPT.FIL

(8) POPT.FIL

(9) NSTRAT.FIL

(10) PSTRAT.FIL

(11) IVCHARN.XXX

(5) NPARIN.FIL
The wafer letter, the row, and the column of the first chip to consider are entered into this file on the first three lines. If the row number is zero, the comprehensive summary is started from scratch. This is done before each lot is analyzed. The fourth and fifth lines should not be modified.

KEYS considers the chips (denoted by wafer letter, (A1,1X,I2, row, and column) in this file.

$1 \mathrm{X}, \mathrm{I} 2)$ The data lines of this file contain the wafer letter,
the row, the column, the width in micrometers of the $n$-channel MOSFETs, the length of the 1X,F5.2,1X, $n$-channel MOSFETs, the width of the p-channel F5.2,1X,F5.2, MOSFETs, the length of the $p$-channel MOSFETs, 1X,F5.2,1X, the measured ring oscillator frequency in $\mathrm{MHz}$, and a designation indicating NMOS technology (1), PMOS technology (2), or CMOS technology (3).

$\mathrm{F} 5.2,1 \mathrm{X}, \mathrm{I} 3)$

(4) LOTNUMBER.FIL The lotnumber is entered into this file.

The n-channel MOSFET oxide thickness in angstroms is followed by the lateral diffusion free format in micrometers.

The p-channel MOSFET oxide thickness in angstroms is followed by the lateral diffusion free format in micrometers.

The $n$-channel MOSFET options file for SUXES. see reference

The $p$-channel MOSFET options file for SUXES. see reference

The $n$-channel MOSFET strategy file for SUXES.

see reference

The $p$-channel MOSFET strategy file for SUXES.

For the $n$-channel MOSFETs, modifications to the SPICE device parameters $(A D, A S, P D, P S$, NRD, and NRS) and output characteristics are made here. The program will input the widths and lengths; therefore, do not modify this portion of the file. 
(12) IVCHARP.XXX For the p-channel MOSFETs, modifications to the SPICE device parameters (AD, AS, PD, PS, NRD, and NRS) and output characteristics are made here. The program will input the widths and lengths; therefore, do not modify this portion of the file.

(13) NMODEL.XXX

For the n-channel MOSFETs, modifications to the SPICE model parameters LEVEL, CJSW, RSH, JS, TPG, MJ, MJSW, PB, and XQC are made here. The program will input the other parameters; therefore, do not modify this portion of the file.

(14) PMODEL.XXX

For the $p$-channel MOSFETs, modifications to the SPICE model parameters LEVEL, CJSW, RSH, JS, TPG, MJ, MJSW, PB, and XQC are made here. The program will input the other parameters; therefore, do not modify this portion of the file.

(15) SUBCKT.XXX

(16) CIRCUIT.FIL

(17) PCHIPS.FIL

Modifications to the SPICE subcircuit file are made here. The program will input the widths and lengths; therefore, do not modify this portion of the file.

Modifications to the chosen SPICE file are made here.

see references $[2,6]$

see references $[2,6]$

see references $[2,6]$

The percentage of acceptable chips needed to make wafer maps is the first data value, and the number of test sites per wafer is the second data value.

(18) STEND.FIL

Modifications to the STAT2 stend array are made here. 


\begin{tabular}{|c|c|c|}
\hline \multirow[t]{3}{*}{$\begin{array}{l}\text { NIST-114A } \\
\text { (REV. 3-90) }\end{array}$} & \multirow{3}{*}{$\begin{array}{l}\text { U.S. DEPARTMENT OF COMMERCE } \\
\text { NATIONAL INSTITUTE OF STANDARDS AND TECHNOLOGY } \\
\text { BIBLIOGRAPHIC DATA SHEET }\end{array}$} & $\begin{array}{l}\text { 1. PUBLCATION OR REPOAT NUMBER } \\
\text { NIST/SP-400/90 }\end{array}$ \\
\hline & & 2. PEAFORMIMG OAGANIZATION REPOAT NUMBEA \\
\hline & & $\begin{array}{l}\text { PUBUCATIONDATE } \\
\text { Apri1 1992 }\end{array}$ \\
\hline
\end{tabular}

4. TITLE AND SUBTITLE

Evaluating a Chip, Wafer, or Lot Using SUXES, SPICE, and STAT2

AUTHOR(S)

J. C. Marshall and R. L. Mattis

6. PERFORMING ORGANIZATION (IF JOINT OR OTHER THAN NIST, SEE INSTRUCTIONS)

U.S. DEPARTMENT OF COMMERCE

MATIONAL INSTITUTE OF STANDARDS AND TECHNOLOQY

QANTHERSBURG, MD 20899

\begin{tabular}{|l|} 
7. \\
\hline CONTRACT/GRANT NUMBER \\
\hline $\begin{array}{l}\text { TYPE OF REPORT AND PERIOD COVERED } \\
\text { Final }\end{array}$
\end{tabular}

9. SPONSORING ORGANIZATION NAME AND COMPLETE ADDRESS (STREET, CITY, STATE, ZIP)

Same as item \#6

10. SUPPLEMENTARY NOTES

11. ABSTRACT (A 200-WOAD OR LESS FACTUAL SUMMARY OF MOST SIGNIFICANT INFORMATION. IF DOCUMENT INCLUDES A SIGNIFICANT BIBUOGAAPHY OR UTERATURE SURVEY, MENTION IT HERE.)

The computer procedure KEYS (linKing softwarE to analYze waferS) links SUXES (Stanford University eXtractor of modEl parameterS), SPICE (a Simulation Program with Integrated Circuit Emphasis), and STAT2. Given data points for individual devices, SUXES obtains the model parameters for SPICE. SPICE predicts the behavior of an individual device or an entire circuit. After analyzing each test chip on a wafer, STAT2 determines correlation coefficients and generates wafer maps of selected parameters. These wafer maps are valuable to the designer, modeler, and process engineer.

The entire package accomplishes the following: (1) standardizes the technique of running SUXES and SPICE in an integrated mode; (2) simulates and plots the characteristic curves; (3) simulates and plots the results of an optional dynamic circuit (for example, a ring oscillator); (4) performs steps (2) and (3) for every test chip on each wafer; (5) summarizes the results from each chip, each wafer, and the lot; (6) rank-orders the model parameters for each wafer according to their correlation coefficients with respect to chosen circuit parameters; and (7) generates wafer maps of several quantities. A CMOS 19-stage ring oscillator is used to illustrate the capabilities of KEYS.

12. KEY WORDS (6 TO 12 ENTRIES; ALPHABETICAL ORDER; CAPITALZE ONLY PAOPER NAMES; AND SEPARATE KEY WORDS BY SEMICOLONS)

circuit simulator; CMOS; ring oscillator, SPICE, STAT2, SUXES, wafer maps

unumite

FOR OFFICIAL DISTRIBUTION. DO NOT RELEASE TO NATIONAL TECHNICAL INFORMATION SERVICE (NTIS).

ORDER FROM SUPERINTENDENT OF DOCUMENTS, U.S. GOVEANMENT PRINTING OFFICE, WASHINGTON, DC 20402.

ORDER FROM MATIONAL TECHMICAL INFORMATION SERVICE (NTIS), SPRINGFIELD, VA 22161.
14. NUMBER OF PRINTED PAGES

145

15. PRICE 




\section{Periodical}

Journal of Research of the National Institute of Standards and Technology-Reports NIST research and development in those disciplines of the physical and engineering sciences in which the Institute is active. These include physics, chemistry, engineering, mathematics, and computer sciences.

Papers cover a broad range of subjects, with major emphasis on measurement methodology and the basic technology underlying standardization. Also included from time to time are survey articles on topics closely related to the Institute's technical and scientific programs. Issued six times a year.

\section{Nonperiodicals}

Monographs-Major contributions to the technical literature on various subjects related to the Institute's scientific and technical activities.

Handbooks - Recommended codes of engineering and industrial practice (including safety codes) developed in cooperation with interested industries, professional organizations, and regulatory bodies.

Special Publications - Include proceedings of conferences sponsored by NIST, NIST annual reports, and other special publications appropriate to this grouping such as wall charts, pocket cards, and bibliographies.

Applied Mathematics Series-Mathematical tables, manuals, and studies of special interest to physicists, engineers, chemists, biologists, mathematicians, computer programmers, and others engaged in scientific and technical work.

National Standard Reference Data Series - Provides quantitative data on the physical and chemical properties of materials, compiled from the world's literature and critically evaluated. Developed under a worldwide program coordinated by NIST under the authority of the National Standard Data Act (Public Law 90-396). NOTE: The Journal of Physical and Chemical Reference Data (JPCRD) is published bimonthly for NIST by the American Chemical Society (ACS) and the American Institute of Physics (AIP). Subscriptions, reprints, and supplements are available from ACS, 1155 Sixteenth St., NW., Washington, DC 20056.

Building Science Series-Disseminates technical information developed at the Institute on building materials, components, systems, and whole structures. The series presents research results, test methods, and performance criteria related to the structural and environmental functions and the durability and safety characteristics of building elements and systems.

Technical Notes-Studies or reports which are complete in themselves but restrictive in their treatment of a subject. Analogous to monographs but not so comprehensive in scope or definitive in treatment of the subject area. Often serve as a vehicle for final reports of work performed at NIST under the sponsorship of other government agencies.

Voluntary Product Standards - Developed under procedures published by the Department of Commerce in Part 10, Title 15, of the Code of Federal Regulations. The standards establish nationally recognized requirements for products, and provide all concerned interests with a basis for common understanding of the characteristics of the products. NIST administers this program as a supplement to the activities of the private sector standardizing organizations.

Consumer Information Series-Practical information, based on NIST research and experience, covering areas of interest to the consumer. Easily understandable language and illustrations provide useful background knowledge for shopping in today's technological marketplace.

Order the above NIST publications from: Superintendent of Documents, Government Printing Office, Washington, DC 20402.

Order the following NIST publications-FIPS and NISTIRs-from the National Technical Information Service, Springfield, VA 22161.

Federal Information Processing Standards Publications (FIPS PUB)-Publications in this series collectively constitute the Federal Information Processing Standards Register. The Register serves as the official source of information in the Federal Government regarding standards issued by NIST pursuant to the Federal Property and Administrative Services Act of 1949 as amended, Public Law 89-306 (79 Stat. 1127), and as implemented by Executive Order 11717 (38 FR 12315, dated May 11, 1973) and Part 6 of Title 15 CFR (Code of Federal Regulations).

NIST Interagency Reports (NISTIR) - A special series of interim or final reports on work performed by NIST for outside sponsors (both government and non-government). In general, initial distribution is handled by the sponsor; public distribution is by the National Technical Information Service, Springfield, VA 2.2161, in paper copy or microfiche form. 


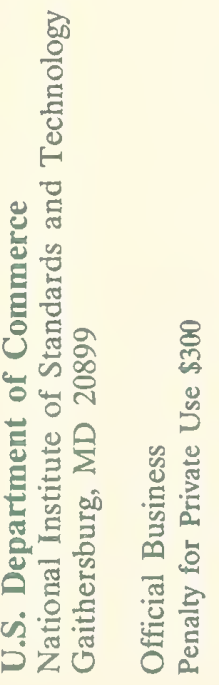

